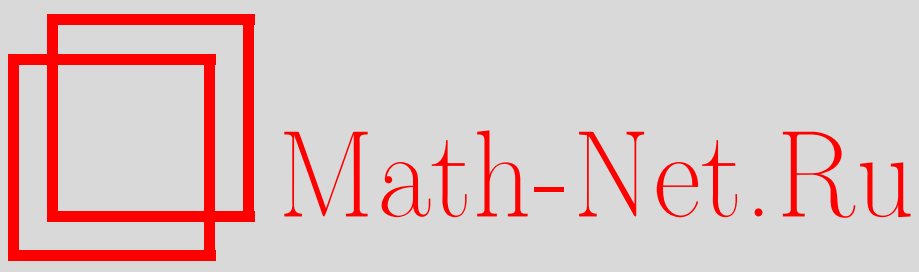

S. K. Vodopyanov, The regularity of inverses to Sobolev mappings and the theory of

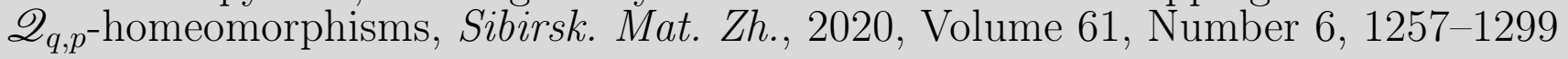

DOI: https://doi.org/10.33048/smzh.2020.61.605

Use of the all-Russian mathematical portal Math-Net.Ru implies that you have read and agreed to these terms of use http://www . mathnet.ru/eng/agreement

Download details:

IP: 44.207 .124 .84

April 26, 2023, 15:37:13 
Сибирский математический журнал Ноябрь-декабрь, 2020. Том 61, № 6

УДК $517.518+517.54$

\title{
О РЕГУЛЯРНОСТИ ОТОБРАЖЕНИЙ, ОБРАТНЫХ К СОБОЛЕВСКИМ, И ТЕОРИЯ $\mathscr{Q}_{q, p}-$ ГОМЕОМОРФИЗМОВ
}

\section{С. К. Водопьянов}

\begin{abstract}
Аннотация. Доказано, что всякий гомеоморфизм $\varphi: D \rightarrow D^{\prime}$ евклидовых областей в $\mathbb{R}^{n}, n \geq 2$, класса Соболева $W_{p, \text { lос }}^{1}(D), p \in[1, \infty)$, с конечным искажением индуцирует ограниченный оператор композиции из весового пространства Соболева $L_{p}^{1}\left(D^{\prime} ; \omega\right)$ в $L_{p}^{1}(D)$ для некоторой весовой функции $\omega: D^{\prime} \rightarrow(0, \infty)$. В качестве следствия отсюда вытекает, что при условиях $p>n-1, n \geq 3$, или $p \geq 1, n \geq 2$, обратный $\varphi^{-1}: D^{\prime} \rightarrow D$ к такому гомеоморфизму принадлежит классу Соболева $W_{1, \text { loc }}^{1}\left(D^{\prime}\right)$, имеет конечное искажение и дифференцируем $\mathscr{H}^{n}$-п. в. в $D^{\prime}$. Получены применения этого результата к теории $\mathscr{Q}_{q, p}$-гомеоморфизмов и обобщен метод его доказательства для гомеоморфизмов групп Карно.

Дополнительно доказано, что класс $\mathscr{Q}_{q, p}$-гомеоморфизмов полностью определяется контролируемым изменением емкости кубических конденсаторов: их оболочки суть концентрические кубы.
\end{abstract}

DOI 10.33048/smzh.2020.61.605

Ключевые слова: квазиконформный анализ, пространство Соболева, оператор композиции, емкостная оценка.

\section{Введение}

Основное содержание работы посвящено доказательству следующей характерной особенности гомеоморфизмов классов Соболева (см. ниже теорему 25) и вытекающих из нее свойств.

Утверждение 1. Если гомеоморфизм $\varphi=\left(\varphi_{1}, \ldots, \varphi_{n}\right): D \rightarrow D^{\prime}, D, D^{\prime} \subset$ $\mathbb{R}^{n}$ - открытые области, $n \geq 2$, принадлежит классу Соболева $W_{p, \text { loc }}^{1}(D), p \in$ $[1, \infty)$, и имеет конечное искажение, то гомеоморфизм $\varphi$ индуцирует ограниченный оператор композиции $\varphi^{*}: L_{p}^{1}\left(D^{\prime} ; \omega\right) \cap \operatorname{Lip}_{l}\left(D^{\prime}\right) \rightarrow L_{p}^{1}(D)$ по правилу $\varphi^{*}(u)=u \circ \varphi, u \in L_{p}^{1}\left(D^{\prime} ; \omega\right) \cap \operatorname{Lip}_{l}\left(D^{\prime}\right)$, с некоторой весовой функцией $\omega: D^{\prime} \rightarrow(0, \infty)$, определяемой ниже в $(30)$.

Напомним, что функция $u: D \rightarrow \mathbb{R}$, определенная на открытом множестве $D \subset \mathbb{R}^{n}$, принадлежит классу Соболева $L_{p}^{1}(D)$, если и локально суммируема в $D$, имеет обобщенные производные $\frac{\partial u}{d x_{j}} \in L_{1, \text { loc }}(D)$ для любого $j=1, \ldots, n$

Работа выполнена при поддержке Математического Центра в Академгородке, соглашение с Министерством науки и высшего образования Российской Федерации (номер 075-152019-1613).

(c) 2020 Водопьянов С. K. 
(т. е. $\frac{\partial u}{d x_{j}} \in L_{1}(U)$ для любой компактно вложенной области $\left.U \Subset D\right)$ и конечную полунорму

$$
\left\|u \mid L_{p}^{1}(D)\right\|=\left(\int_{D}|\nabla u(y)|^{p} d y\right)^{\frac{1}{p}}, \quad 1 \leq p \leq \infty .
$$

Здесь и далее символом $\operatorname{Lip}_{l}\left(D^{\prime}\right)$ обозначено пространство локально липшицевых функций, определенных на области $D^{\prime}$. Очевидно, $\operatorname{Lip}_{l}\left(D^{\prime}\right)=W_{\infty, \mathrm{loc}}^{1}\left(D^{\prime}\right) \cap$ $C\left(D^{\prime}\right)$.

Отображение $\varphi=\left(\varphi_{1}, \ldots, \varphi_{n}\right)$ принадлежит классу Соболева $W_{p, \mathrm{loc}}^{1}(D)$, если $\varphi_{j}(x)$ и обобщенные производные $\frac{\partial \varphi_{j}}{d x_{i}}$ принадлежат $L_{p, \operatorname{loc}}(D)$ для любых $j, i=1, \ldots, n$.

Отображение $\varphi: D \rightarrow \mathbb{R}^{n}$ класса Соболева $W_{1, \mathrm{loc}}^{1}(D)$ называют отображением с конечным искажением, если

$$
D \varphi(x)=0 \quad \mathscr{H}^{n} \text {-п. в. на множестве } Z=\{x \in D: \operatorname{det} D \varphi(x)=0\} .
$$

Здесь и далее $D \varphi(x)=\left(\frac{\partial \varphi_{j}}{\partial x_{i}}(x)\right)$ - матрица Якоби отображения $\varphi$ в точке $x \in$ $D,|D \varphi(x)|$ - ее евклидова операторная норма, a $\operatorname{det} D \varphi(x)$ - ее определитель (якобиан).

Сформулированное выше утверждение 1 о функциональной характеристике гомеоморфизма класса Соболева служит основой для доказательства новых свойств обратного гомеоморфизма (см. ниже теорему 27).

Утверждение 2. Если гомеоморфизм $\varphi=\left(\varphi_{1}, \ldots, \varphi_{n}\right): D \rightarrow D^{\prime}, D, D^{\prime} \subset$ $\mathbb{R}^{n}$ - открытые области, $n \geq 2$, принадлежит классу Соболева $W_{p, \mathrm{loc}}^{1}(D), p \in$ $[1, \infty)$ при $n=2$ или $p \in(n-1, \infty)$ при $n \geq 3$, и имеет конечное искажение, то обратный гомеоморфизм $\varphi^{-1}: D^{\prime} \rightarrow D$

1) принадлежит классу Соболева $W_{1, \mathrm{loc}}^{1}\left(D^{\prime}\right)$,

2) имеет конечное искажение,

3) дифференцируем $\mathscr{H}^{n}-$ п. в. в $D^{\prime}$.

Заметим, что утверждения 1,2 доказаны в [1, теоремы $3.2,3.3]$ при $n=2$, $p=1$ и в [2, теорема 1.2] при $n \geq 3, p>n-1$; работы [1,2] мотивированы применениями к задачам нелинейной теории упругости (см. [3]); утверждение 3 можно получить из [4; 5 , теорема 2] при $n=2, p=1$ и из монографии [6] при $n \geq 3, p=n$, в которой приведена также подробная библиография.

Доказательство свойств, сформулированных в утверждении 2 (см. теорему 27), новое и более лаконичное по сравнению с доказательствами вышеперечисленных работ.

Более того, новое доказательство применимо и на метрических структурах более сложной природы (см. ниже $\S 4$, где установлены аналоги утверждений 1 и 2 на группах Карно).

Настоящая статья естественно входит в цикл публикаций [7-12], начавшийся в ряде работ [13-20] и возникший на стыке теории функциональных пространства и геометрической теории функций [21-39]. Некоторые результаты упомянутых работ нашли применения в нелинейной теории упругости (см. [40]).

Основные результаты настоящей работы сформулированы в [11].

\section{$\S 1$. Предварительные сведения}

Сформулируем основной результат работ [10,12]. Можно сказать, что он представляет собой «весовое» обобщение результатов публикаций [13-16] (соответственно [17-20]) в части $1<q=p<\infty(1<q<p<\infty)$. 
Теорема 3 [10,12]. Пусть заданы гомеоморфизм $\varphi: D \rightarrow D^{\prime}$ областей $D, D^{\prime} \subset \mathbb{R}^{n}, n \geq 2$, и весовая локально суммируемая функция $\omega: D^{\prime} \rightarrow(0, \infty)$.

Следующие условия эквивалентны:

1) оператор композиции $\varphi^{*}: L_{p}^{1}\left(D^{\prime} ; \omega\right) \cap \operatorname{Lip}_{l}\left(D^{\prime}\right) \rightarrow L_{q}^{1}(D), 1 \leq q \leq p<\infty$, ограничен;

2) для любого кольцевого ${ }^{1)}$ конденсатора $E=(F, U)$ в $D^{\prime}$ с прообразом $\varphi^{-1}(E)=\left(\varphi^{-1}(F), \varphi^{-1}(U)\right)$ в $D$ выполняется неравенство (здесь и далее число $\sigma$ определяется соотношениями $\frac{1}{\sigma}=\frac{1}{q}-\frac{1}{p}$, если $1 \leq q<p<\infty$, и $\sigma=\infty$, если $1 \leq q=p<\infty)$

$$
\operatorname{cap}^{\frac{1}{q}}\left(\varphi^{-1}(E) ; L_{q}^{1}(D)\right) \leq \begin{cases}K_{p} \operatorname{cap}^{\frac{1}{p}}\left(E ; L_{p}^{1}\left(D^{\prime} ; \omega\right)\right), & 1<q=p<\infty \\ \Psi_{q, p}(U \backslash F)^{\frac{1}{\sigma}} \operatorname{cap}^{\frac{1}{p}}\left(E ; L_{p}^{1}\left(D^{\prime} ; \omega\right)\right), & 1<q<p<\infty\end{cases}
$$

где $\Psi_{q, p}$ - некоторая ограниченная квазиаддитивная функция множества, определенная на открытых подмножествах области $D^{\prime}$;

3) гомеоморфизм $\varphi: D \rightarrow D^{\prime}$ принадлежит $W_{q, \mathrm{loc}}^{1}(D)$, имеет конечное искажение: $D \varphi(x)=0 \mathscr{H}^{n}$-п. в. на множестве $Z \stackrel{q,}{=}\{x \in D \mid J(x, \varphi)=0\}$, и операторная функция искажения

$$
D \ni x \mapsto K_{q, p}^{1, \omega}(x, \varphi)= \begin{cases}\frac{|D \varphi(x)|}{|\operatorname{det} D \varphi(x)|^{\frac{1}{p}} \omega^{\frac{1}{p}}(\varphi(x))}, & \text { если } \operatorname{det} D \varphi(x) \neq 0, \\ 0, & \text { если } \operatorname{det} D \varphi(x)=0,\end{cases}
$$

принадлежит $L_{\sigma}(D)$.

Кроме того, $\varphi \in W_{q, \mathrm{loc}}^{1}(D)$ и

$$
\begin{aligned}
& 2^{-\frac{n}{q}}\left(\frac{3 n}{2}\right)^{-1}\left\|K_{q, p}^{1, \omega}(\cdot) \mid L_{\sigma}(D)\right\| \leq\left\|\varphi^{*}\right\| \\
& \leq\left\|K_{q, p}^{1, \omega}(\cdot) \mid L_{\sigma}(D)\right\| \leq \begin{cases}3 n 2^{\frac{n-p}{p}} K_{p} & \text { при } q=p, \\
3 n 2^{\frac{n-q}{q}} \Psi_{q, p}\left(D^{\prime}\right)^{\frac{1}{\sigma}} & \text { при } q<p .\end{cases}
\end{aligned}
$$

ЗАмечАниЕ 4. Эквивалентность утверждений 1-3 теоремы 3 доказана в $[10,12]$ при $1<q \leq p<\infty$, что обусловлено пределами изменения параметров суммируемости в $(2)$.

В то же время эквивалентность утверждений 1 и 3 теоремы 3 доказана в $[10,12,41,42]$ при $1 \leq q \leq p<\infty$. В этой работе установлено (см. ниже следствие 29), что все утверждения теоремы 3 эквивалентны также и при $1=$ $q \leq p<\infty, n=2$.

Действительно, импликация $1 \Rightarrow 2$ в теореме 3 доказана в $[10,12]$ при $1<q$, однако то же самое доказательство работает и при $n=2, q=1$. Импликация $2 \Rightarrow 3$ при $q=1, n=2$ доказана в следствии 29 настоящей работы.

Приведем определения всех используемых в этой теореме понятий.

Локально суммируемая функция $\omega: D^{\prime} \rightarrow \mathbb{R}$ называется весовой, если $0<\omega(y)<\infty$ для $\mathscr{H}^{n}$-п. в. $y \in D^{\prime}$. Напомним, что функция $u: D^{\prime} \rightarrow \mathbb{R}$ принадлежит весовому классу Соболева $L_{p}^{1}\left(D^{\prime} ; \omega\right), p \in[1, \infty)$, если $u$ локально суммируема в $D^{\prime}$, а обобщенные производные ${ }^{2)} \frac{\partial u}{\partial y_{j}}$ принадлежат $L_{p}\left(D^{\prime} ; \omega\right)$ для

\footnotetext{
1) Описание кольцевого конденсатора см. ниже в определении 5.

2) Определение обобщенных производных предполагает, что $\frac{\partial u}{d y_{j}} \in L_{1, \text { loc }}\left(D^{\prime}\right)$.
} 
любого $j=1, \ldots, n$. Полунорма функции $u \in L_{p}^{1}\left(D^{\prime} ; \omega\right)$ равна

$$
\left\|u \mid L_{p}^{1}\left(D^{\prime} ; \omega\right)\right\|=\left(\int_{D^{\prime}}|\nabla u|^{p}(y) \omega(y) d y\right)^{\frac{1}{p}} .
$$

В случае $\omega \equiv 1$ вместо $L_{p}^{1}\left(D^{\prime} ; 1\right)$ пишем просто $L_{p}^{1}\left(D^{\prime}\right)$.

Напомним, что гомеоморфизм $\varphi: D \rightarrow D^{\prime}$ порождает ограниченный оператор композиции

$$
\varphi^{*}: L_{p}^{1}\left(D^{\prime} ; \omega\right) \cap \operatorname{Lip}_{l}\left(D^{\prime}\right) \rightarrow L_{q}^{1}(D), \quad 1 \leq q \leq p<\infty,
$$

действующий по правилу $D \ni x \mapsto\left(\varphi^{*} u\right)(x)=u(\varphi(x))$, если с некоторой постоянной $K_{q, p}<\infty$ справедливо неравенство

$\left\|\varphi^{*} u\left|L_{q}^{1}(D)\left\|\leq K_{q, p}\right\| u\right| L_{p}^{1}\left(D^{\prime} ; \omega\right)\right\|$ для любой функции $u \in L_{p}^{1}\left(D^{\prime}\right) \cap \operatorname{Lip}_{l}\left(D^{\prime}\right)$.

ОПРЕДЕЛЕНиЕ 5. Конденсатором в области $D^{\prime} \subset \mathbb{R}^{n}$ называется пара $E=\left(F_{1}, F_{0}\right)$ связных компактов (континуумов) в $D^{\prime}: F_{1}, F_{0} \subset D^{\prime}$. Если континуум $F$ содержится в $U$, где $U \Subset D^{\prime}$ - открытое связное компактно вложенное множество, то конденсатор $E=(F, \partial U)$ будем обозначать символом $E=(F, U)$.

Конденсатор $E=(F, \partial U)$ называется кольцевым, если дополнение в $\mathbb{R}^{n}$ к открытому множеству $U \backslash F$ состоит из двух замкнутых множеств, каждое из которых связно: ограниченная компонента связности - континуум $F$, а неограниченная $-\mathbb{R}^{n} \backslash U$.

Кольцевой конденсатор $E=(F, \partial U)$ в $\mathbb{R}^{n}$ называется сферическим (кубическим), если область $U$ - это шар ${ }^{3)} B(x, R)=\left\{y \in \mathbb{R}^{n}:|y-x|_{2}<R\right\}$ (куб $\left.Q(x, R)=\left\{y \in \mathbb{R}^{n}:|y-x|_{\infty}<R\right\}\right)$, а континуум $F \subset U$ - это замыкание шара $B(x, r)=\left\{y \in \mathbb{R}^{n}:|y-x|_{2} \leq r\right\}$ (куба $Q(x, r)=\left\{y \in \mathbb{R}^{n}:|y-x|_{\infty}<r\right\}$ ), где $r<R$.

Непрерывная функция $u: D \rightarrow \mathbb{R}$ класса $W_{1, \text { loc }}^{1}(D)$ называется допустимой для конденсатора $E=\left(F_{1}, F_{0}\right) \subset D$, если $u \equiv 1$ на $F_{1}$ и $u \equiv 0$ на $F_{0}$. Совокупность допустимых для конденсатора $E=\left(F_{1}, F_{0}\right)$ функций будем обозначать символом $\mathscr{A}(E)$.

Емкость конденсатора $E=\left(F_{1}, F_{0}\right)$ в пространстве $L_{q}^{1}(D), q \in[1, \infty)$, определим как величину

$$
\operatorname{cap}\left(E ; L_{q}^{1}(D)\right)=\inf _{u \in \mathscr{A}(E)}\left\|u \mid L_{q}^{1}(D)\right\|^{q},
$$

где инфимум берется по всем допустимым для конденсатора $E=\left(F_{1}, F_{0}\right) \subset D$ функциям класса $\mathscr{A}(E)$.

Весовую емкость конденсатора $E=\left(F_{1}, F_{0}\right) \subset D^{\prime}$ в пространстве $L_{p}^{1}\left(D^{\prime} ; \omega\right)$ определим как величину

$$
\operatorname{cap}\left(E ; L_{p}^{1}\left(D^{\prime} ; \omega\right)\right)=\inf _{u \in \mathscr{A}(E) \cap \operatorname{Lip}_{l}\left(D^{\prime}\right)}\left\|u \mid L_{p}^{1}\left(D^{\prime} ; \omega\right)\right\|^{p},
$$

где инфимум берется по всем допустимым для конденсатора $E=\left(F_{1}, F_{0}\right)$ функциям пересечения $\mathscr{A}(E) \cap \operatorname{Lip}_{l}\left(D^{\prime}\right)$.

Далее рассматриваем в области $D^{\prime}$ преимущественно кольщевые конденсаторы вида $E=(F, U)$.

\footnotetext{
3) Напомним, что норма $|x|_{p}$ вектора $x=\left(x_{1}, x_{2}, \ldots, x_{n}\right) \in \mathbb{R}^{n}$ определяется как величина $|x|_{p}=\left(\sum_{k=1}^{n}\left|x_{k}\right|^{p}\right)^{\frac{1}{p}}$ при $p \in[1, \infty)$ и $|x|_{\infty}=\max _{k=1, \ldots, n}\left|x_{k}\right|$. Шары в норме $|x|_{2}\left(|x|_{\infty}\right)$ - это (евклидовы) шары (кубы).
} 
ОПРеДЕЛЕНИЕ 6 . Пусть $D$ - открытое множество в $\mathbb{R}^{n}$. Обозначим символом $\mathscr{O}(D)$ некоторую систему открытых множеств в $D$, обладающую следующими свойствами:

(а) если замыкание $\bar{B}$ открытого шара ${ }^{4)} B$ содержится в $D$, то $B \in \mathscr{O}(D)$;

(b) если $U_{1}, \ldots, U_{k} \in \mathscr{O}(D)$ - дизъюнктная система открытых множеств, то $\bigcup_{i=1}^{k} U_{i} \in \mathscr{O}(D), k \in \mathbb{N}-$ произвольное число.

Отображение $\Phi: \mathscr{O}(D) \rightarrow[0, \infty]$ называется $\varkappa$-квазиаддитивной функцией множества, если

(c) для всякой точки $x \in D$ существует $\delta, 0<\delta<\operatorname{dist}(x, \partial D)$, такое, что $0<\Phi(B(x, \delta))<\infty$ (если $D=\mathbb{R}^{n}$, то неравенство $0 \leq \Phi(D(x, \delta))<\infty$ должно выполняться для всех $\delta \in(0, \delta(x))$, где $\delta(x)>0$ - число, которое может зависеть от точки $x)$;

(d) для всякого конечного дизъюнктного набора $U_{i} \in \mathscr{O}(D), i=1, \ldots, l$, открытых множеств таких, что

$$
\bigcup_{i=1}^{l} U_{i} \subset U, \text { где } U \in \mathscr{O}(D) \text {, верно неравенство } \sum_{i=1}^{l} \Phi\left(U_{i}\right) \leq \varkappa \Phi(U) .
$$

Если неравенство (7) выполняется с постоянной $\varkappa=1$, то $\Phi$ будем называть квазиаддитивной (вместо 1-квазиаддитивной) функиией множества.

Если для всякого конечного набора $\left\{U_{i} \in \mathscr{O}(D)\right\}$ попарно не пересекающихся открытых множеств имеет место равенство

$$
\sum_{i=1}^{n} \Phi\left(U_{i}\right)=\Phi\left(\bigcup_{i=1}^{n} U_{i}\right)
$$

то такая функция множества называется конечно аддитивной, а если (8) справедливо для всякого счетного набора $\left\{U_{i} \in \mathscr{O}(D)\right\}$ попарно не пересекающихся открытых множеств, то - счетно аддитивной.

Функция $\Phi$ монотонна, если $\Phi\left(U_{1}\right) \leq \Phi\left(U_{2}\right)$ при условии, что $U_{1} \subset U_{2} \subset D$, $U_{1}, U_{2} \in \mathscr{O}(D)$. Очевидно, что всякая квазиаддитивная функция множества монотонна.

$\varkappa$-Квазиаддитивная функция множества $\Phi: \mathscr{O}(D) \rightarrow[0, \infty]$ называется ограниченной, если $\sup _{U \in \mathscr{O}(D)}<\infty$.

Теорема 3 мотивирует выделение в самостоятельный объект исследования следующую шкалу отображений. Напомним, что отображение $f: D^{\prime} \rightarrow \mathbb{R}^{n}, D^{\prime}$ - область в $\subset \mathbb{R}^{n}$, называется непрерывным открытым и дискретным, если $f$ непрерывно в $D^{\prime}$, образ открытого множества открыт и прообраз $f^{-1}(y)$ точки $y \in f(D)$ дискретен.

ОПРЕДЕЛЕНИЕ 7 [10,12]. Будем говорить, что гомеоморфизм (или, более общо, непрерывное открытое и дискретное отображение) $f: D^{\prime} \rightarrow D, D, D^{\prime} \subset$ $\mathbb{R}^{n}, n \geq 2$, принадлежсит классу $\mathscr{Q}_{q, p}\left(D^{\prime}, D ; \omega\right)$, где $1<q \leq p<\infty$ при $n \geq 3$ или $1 \leq q \leq p<\infty$ при $n=2$, а $\omega \in L_{1, \text { loc }}\left(D^{\prime}\right)$ - весовая функция, если существуют

1) постоянная $K_{p}$ при $q=p$

или

$2)$ ограниченная квазиаддитивная функция $\Psi_{q, p}$ при $q<p$, заданная на открытых множествах в $D^{\prime}$,

4) В качестве элементарных множеств вместо шаров можно рассматривать кубы. 
такие, что для всякого конденсатора $E=\left(F_{0}, F_{1}\right)$, расположенного в $D^{\prime}$, и образа $f(E)=\left(f\left(F_{0}\right), f\left(F_{1}\right)\right)$, расположенного в $D$, выполняются неравенства

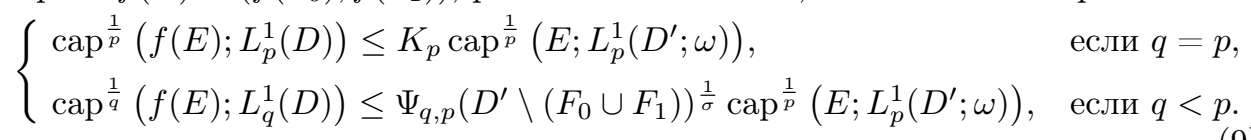

Если условия (9) выполняются только для кольцевых конденсаторов $E=$ $(F, U) \subset D^{\prime}:$

$$
\begin{cases}\operatorname{cap}^{\frac{1}{p}}\left(f(E) ; L_{p}^{1}(D)\right) \leq K_{p} \operatorname{cap}^{\frac{1}{p}}\left(E ; L_{p}^{1}\left(D^{\prime} ; \omega\right)\right), & \text { если } q=p, \\ \operatorname{cap}^{\frac{1}{q}}\left(f(E) ; L_{q}^{1}(D)\right) \leq \Psi_{q, p}(U \backslash F)^{\frac{1}{\sigma}} \operatorname{cap}^{\frac{1}{p}}\left(E ; L_{p}^{1}\left(D^{\prime} ; \omega\right)\right), & \text { если } q<p,\end{cases}
$$

то получаем более широкий класс гомеоморфизмов $f: D^{\prime} \rightarrow D$ и обозначаем его символом $\mathscr{R} \mathscr{Q}_{q, p}\left(D^{\prime}, D ; \omega\right)$.

Если условия (10) выполняются только для сферических (кубических) кольцевых конденсаторов, то получаем еще более широкий класс гомеоморфизмов $f: D^{\prime} \rightarrow D$ и обозначаем его символом $S \mathscr{R} Q_{q, p}\left(D^{\prime}, D ; \omega\right)\left(Q \mathscr{R} Q_{q, p}\left(D^{\prime}, D ; \omega\right)\right)$.

Очевидно,

$$
\mathscr{Q}_{q, p}\left(D^{\prime}, D ; \omega\right) \subset \mathscr{R} Q_{q, p}\left(D^{\prime}, D ; \omega\right) \subset S \mathscr{R} Q_{q, p}\left(D^{\prime}, D ; \omega\right) \quad\left(Q \mathscr{R} Q_{q, p}\left(D^{\prime}, D ; \omega\right)\right) .
$$

ЗАмечание 8 . В случае $q=p=n$ класс гомеоморфизмов $\mathscr{Q}_{n, n}\left(D^{\prime}, D ; \omega\right)$ содержит [12, разд. 4.4] класс так называемых $Q$-гомеоморфизмов [6], определяемых посредством контролируемого изменения модуля семейств кривых.

В следующей теореме приводится аналитическое описание отображений, обратные к которым принадлежат классу $\mathscr{Q}_{q, p}\left(D^{\prime}, D ; \omega\right)$.

Теорема $9[10,12]$. Гомеоморфизм $f: D^{\prime} \rightarrow D$ тогда и только тогда принадлежит классу $\mathscr{R} Q_{q, p}\left(D^{\prime}, D ; \omega\right), 1<q \leq p<\infty$ при $n \geq 3$ и $1 \leq q \leq p<\infty$ при $n=2$, когда обратный гомеоморфизм $\varphi=f^{-1}: D \rightarrow D^{\prime}$ обладает либо свойством 1, либо свойством 3 теоремы 3.

ДоказАтельство. Нетрудно заметить, что условие $f \in \mathscr{R} Q_{q, p}\left(D^{\prime}, D ; \omega\right)$, $1<q \leq p<\infty, 2 \leq n$, для гомеоморфизма $f: D^{\prime} \rightarrow D$ эквивалентно выполнению условия 2 в теореме 3 для обратного гомеоморфизма $\varphi=f^{-1}: D \rightarrow D^{\prime}$. Отсюда выводим, что для отображения $\varphi: D \rightarrow D^{\prime}$ выполнены утверждение 1 и утверждение 3 теоремы 3. Так как приведенные рассуждения обратимы, теорема 9 доказана в случае $n>2$. Справедливость теоремы 9 в случае $1=q \leq p<\infty, n=2$, доказана в теореме 3 , замечании 4 и следствии 29 настоящей работы.

В этой работе приведены новые примеры классов отображений, входящих в семейство $\mathscr{Q}_{q, p}\left(D^{\prime}, D ; \omega\right)$.

ЗАмечание 10. В [13-16], доказано, что в случае $1<q=p<\infty, \omega \equiv 1$ оператор композиции $\varphi^{*}: L_{p}^{1}\left(D^{\prime}\right) \cap \operatorname{Lip}_{l}\left(D^{\prime}\right) \rightarrow L_{p}^{1}(D)$ теоремы 3 распространяется по непрерывности на пространство $L_{p}^{1}\left(D^{\prime}\right)$ и совпадает с оператором композиции в следующем смысле:

$$
L_{p}^{1}\left(D^{\prime}\right) \ni u \mapsto \varphi^{*} u= \begin{cases}u \circ \varphi, & \text { где } u-\text { непрерывный представи- } \\ & \text { тель } u \in L_{p}^{1}\left(D^{\prime}\right) \text { при } p \in(n, \infty), \\ u \circ \varphi, & \text { где } u-\text { произвольный представи- } \\ & \text { тель } u \in L_{p}^{1}\left(D^{\prime}\right) \text { при } p \in[1, n] .\end{cases}
$$

При $p=n$ отображения этого класса квазиконформные. В [43] отображения этого класса при $p \neq n$ названы $p$-морфизмами. 
Напомним несколько полезных понятий. Для $k \geq 0, \delta \in(0, \infty]$ и $A \subset \mathbb{R}^{n}$ определим величину

$$
\mathscr{H}_{\delta}^{k}(A)=\frac{\omega_{k}}{2^{k}} \inf \left\{\sum_{i \in \mathbb{N}}\left(\operatorname{diam} T_{i}\right)^{k}: \operatorname{diam} T_{i}<\delta, A \subset \bigcup_{i \in \mathbb{N}} T_{i}\right\},
$$

где $\omega_{k}=\frac{\frac{k}{2}}{\Gamma\left(\frac{k}{2}+1\right)}$, а инфимум берется по всем счетным покрытиям $\left\{T_{i}\right\}$ множества $A$. Если $A$ не может быть покрыто счетным набором множеств указанного размера, то полагаем $\mathscr{H}_{\delta}^{k}(A)=\infty$. Предел $\mathscr{H}^{k}(A)=\lim _{\delta \rightarrow 0} \mathscr{H}_{\delta}^{k}(A)$ существует и называется $k$-мерной мерой Хаусдорфа множества $A$. В евклидовом пространстве $\mathbb{R}^{n} n$-мерная мера Хаусдорфа $\mathscr{H}^{n}(A)$ множества $A \subset \mathbb{R}^{n}$ совпадает с $n$-мерной мерой Лебега (см., например, [44, теорема 2.3.4]).

Квазиаддитивная функция множества $\Phi$, определенная выше, дифференцируема в следующем смысле.

Предложение 11 [45-47]. I. Пусть монотонная квазиаддитивная функция множества $\Phi$ определена на некоторой системе $\mathscr{O}\left(D^{\prime}\right)$ открытых подмножествах области $D^{\prime}$. Тогда

1) для $\mathscr{H}^{n}$-п. в. точек $y \in D^{\prime}$ существует конечная производная ${ }^{5)}$ :

$$
\lim _{\delta \rightarrow 0, y \in B_{\delta}} \frac{\Phi\left(B_{\delta}\right)}{\mathscr{H}^{n}\left(B_{\delta}\right)}=\Phi^{\prime}(y) ;
$$

2) для любого открытого множества $U \in \mathscr{O}\left(D^{\prime}\right)$ справедливо неравенство

$$
\int_{U} \Phi^{\prime}(y) d y \leq \Phi(U)
$$

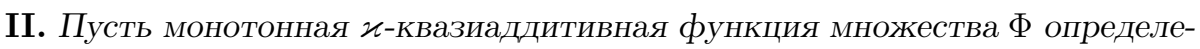
на на некоторой системе $\mathscr{O}\left(D^{\prime}\right)$ открытых подмножествах области $D^{\prime}$. Тогда

3) для $\mathscr{H}^{n}$-п. в. точек $y \in D^{\prime}$ существует конечная верхняя производная:

$$
\lim _{r \rightarrow 0} \sup _{0<\delta<r, y \in B_{\delta}} \frac{\Phi\left(B_{\delta}\right)}{\mathscr{H}^{n}\left(B_{\delta}\right)}=\bar{\Phi}^{\prime}(y) ;
$$

4) для любого открытого множества $U \in \mathscr{O}\left(D^{\prime}\right)$ справедливо неравенство

$$
\int_{U} \bar{\Phi}^{\prime}(y) d y \leq \varkappa \Phi(U) .
$$

Во всех пределах шары можно заменить кубами.

ПРИМЕР 12 (ОБЪЕМНАЯ ПРОИЗВОДНАЯ). І. Пусть $D^{\prime}$ - открытое множество в $\mathbb{R}^{n}$, а $f: D^{\prime} \rightarrow \mathbb{R}^{n}$ - инъективное непрерывное отображение. Для любого открытого множества $U \subset D^{\prime}$ образ $f(U)$ является борелевским множеством и поэтому определена функция множества $\mathscr{V}_{n}$ :

$$
U \mapsto \mathscr{V}_{n}(U)=\mathscr{H}^{n}(f(U))
$$

Функция $\mathscr{V}_{n}$ определена на открытых множествах $U \subset D^{\prime}$ и является, очевидно, монотонной и счетно аддитивной. В силу предложения 11 существует производная $\mathscr{V}_{n}^{\prime}(y)$, совпадающая для $\mathscr{H}^{n}$-п. в. $y \in D^{\prime}$ с плотностью (объемной производной)

$$
D^{\prime} \ni y \mapsto J_{f}(y)=\lim _{r \rightarrow 0} \frac{\mathscr{H}^{n}(f(B(y, r)))}{\mathscr{H}^{n}(B(y, r))}
$$

\footnotetext{
5) Здесь и далее $B_{\delta}-$ произвольный шар $B(z, \delta) \subset D^{\prime}$, содержащий точку $y$.
} 
функции множества $\mathscr{B}\left(D^{\prime}\right) \ni T \mapsto \mathscr{H}^{n}(f(T))$, определенной на $\sigma$-алгебре $\mathscr{B}\left(D^{\prime}\right)$ борелевских множеств $T \subset D^{\prime}$.

II. Пусть $D^{\prime}$ - открытое множество в $\mathbb{R}^{n}$, а $f: D^{\prime} \rightarrow \mathbb{R}^{n}$ - непрерывное открытое дискретное отображение. Фиксируем произвольное открытого множества $U \Subset D^{\prime}$. Его образ $f(U)$ является открытым ограниченным множеством. Более того, функция кратности (индикатриса Банаха)

$$
f(U) \ni x \mapsto \mathscr{N}(x, f, U)=\#\{y \in U: f(y)=x\}
$$

ограничена (см. [48, предложение 4.1]). Положим $\mathscr{N}(f, U)=\sup _{x \in f(U)} \mathscr{N}(x, f, U)$.

Имеем $\mathscr{N}(f, U)<\infty$.

Функция множества $\mathscr{V}_{n}$ :

$$
U \supset V \mapsto \mathscr{V}_{n}(V)=\mathscr{H}^{n}(f(V)),
$$

определенная на открытых множествах $V \subset U$, является монотонной $\varkappa$-квазиаддитивной функцией множества с постоянной $\varkappa=\mathscr{N}(f, U)$. Действительно, если $V_{i} \subset U, i=1, \ldots, l,-$ конечный дизъюнктный набор открытых множеств, то

$$
\begin{aligned}
\sum_{i=1}^{l} \mathscr{V}_{n}\left(V_{i}\right)=\sum_{i=1}^{l} \mathscr{H}^{n}\left(f\left(V_{i}\right)\right)=\sum_{i=1}^{l} \int_{f(U)} \chi_{f\left(V_{i}\right)}(x) d x & \\
& \leq \int_{f(U)} \mathscr{N}(f, U) d x=\mathscr{N}(f, U) \mathscr{V}_{n}(U) .
\end{aligned}
$$

В силу предложения 11 существует верхняя производная $\overline{\mathscr{V}}_{n}^{\prime}(y)$ :

$$
U \ni y \mapsto \overline{\mathscr{V}}_{n}^{\prime}(y)=\varlimsup_{r \rightarrow 0, y \in B_{r}} \frac{\mathscr{H}^{n}\left(\mathscr{V}_{n}\left(B_{r}\right)\right)}{\mathscr{H}^{n}\left(B_{r}\right)}=\varlimsup_{r \rightarrow 0, y \in B_{r}} \frac{\mathscr{H}^{n}\left(f\left(B_{r}\right)\right)}{\mathscr{H}^{n}\left(B_{r}\right)},
$$

где $B_{r}$ - шар радиуса $r$, содержащий точку $y$, не обязательно совпадающую с центром шара $B_{r}$. При этом по предложению 11 верно неравенство

$$
\int_{V} \overline{\mathscr{V}}_{n}^{\prime}(y) d y \leq \varkappa \mathscr{V}_{n}(V)
$$

для любого открытого множества $V \subset U$.

ПРИМЕР 13 (ТЕОРЕМА ЛЕБЕГА О ДИФФЕРЕНЦИРОВАНИИ ИНТЕГРАЛА). Пусть $D$ - открытое множество в $\mathbb{R}^{n}$, а $g \in L_{1, \text { lос }}(D)$ - неотрицательная функция. Для открытого множества $U \subset D$ положим $\Phi(U)=\int_{U} g(x) d x$. Функция $\Phi$ определена на открытых множествах $U \subset D$, монотонна и счетно аддитивна. Ее производная $\Phi^{\prime}(x)$ существует для $\mathscr{H}^{n}$-п. в. $x \in D$ и совпадает $\mathscr{H}^{n}$-п. в. с функцией $g(x)[49,50]$.

Напомним, что функция $u: D \rightarrow \mathbb{R}$ принадлежит $\mathrm{ACL}(D)(u \in \operatorname{ACL}(D))$, если ограничение $\left.u\right|_{Q}$ функции $u: D \rightarrow \mathbb{R}$ на любой замкнутый куб $Q \subset D$, ребра которого параллельны координатным осям, абсолютно непрерывно на $\mathscr{H}^{n-1}$-п. в. отрезках, перпендикулярных граням этого куба. Известно (см., например, [51]), что всякую функцию класса $f \in W_{1, \text { loc }}^{1}(D)$ можно переопределить на множестве меры нуль так, что переопределенная функция $\tilde{f}$ будет принадлежать $\mathrm{ACL}(D)$, а все ее частные производные будут совпадать с обобщенными $\mathscr{H}^{n}$-п. в. в $D$.

Ниже будем применять следующую формулу о замене переменной в интеграле Лебега. 
Предложение 14 [12,52]. Пусть $\varphi: D \rightarrow \mathbb{R}^{n}$ - отображение класса Соболева $W_{1, \text { loc }}^{1}(D)$ (или класса $\left.\mathrm{ACL}(D)\right)$. Тогда

1) существует борелевское множество $\Sigma \subset D$ нулевой меры такое, что $\varphi$ : $D \backslash \Sigma \rightarrow \mathbb{R}^{n}$ обладает $\mathscr{N}$-свойством Лузина;

2) функции

$$
D \backslash \Sigma \ni x \mapsto(u \circ \varphi)(x)|\operatorname{det} D \varphi(x)| \quad \text { и } \quad \mathbb{R}^{n} \ni y \mapsto u(y) \mathscr{N}(y, \varphi, D \backslash \Sigma)
$$

измеримы, если функция $u: \mathbb{R}^{n} \rightarrow \mathbb{R}$ измерима;

3) если $A \subset D \backslash \Sigma-$ измеримое множество, то верна формула площади:

$$
\int_{A}|\operatorname{det} D \varphi(x)| d x=\int_{\mathbb{R}^{n}} \mathscr{N}(y, \varphi, A) d y ;
$$

4) если функция $u \geq 0$ неотрицательна, то подынтегральные функции в (13) измеримы и верна следующая формула замены переменной в интеграле Лебега:

$$
\int_{D \backslash \Sigma} u(\varphi(x))|\operatorname{det} D \varphi(x)| d x=\int_{\mathbb{R}^{n}} \sum_{x \in \varphi^{-1}(y) \backslash \Sigma} u(x) d y ;
$$

5) если одна из функций

$$
D \backslash \Sigma \ni x \mapsto(u \circ \varphi)(x)|\operatorname{det} D \varphi(x)| \quad \text { или } \quad \mathbb{R}^{n} \ni y \mapsto u(y) \mathscr{N}(y, \varphi, D \backslash \Sigma)
$$

интегрируема, то и другая интегрируема и верна формула

$$
\int_{D \backslash \Sigma} u(\varphi(x))|\operatorname{det} D \varphi(x)| d x=\int_{\mathbb{R}^{n}} u(y) \mathscr{N}(y, \varphi, D \backslash \Sigma) d y .
$$

ЗАмечание 15. Так как $\mathscr{H}^{n}(\Sigma)=0$ в формулах (13) и (14), то в левых частях этих формул интегрирование по $D \backslash \Sigma$ можно заменить интегрированием по $D$, так что вместе с (14) верна также формула

$$
\int_{D} u(\varphi(x))|\operatorname{det} D \varphi(x)| d x=\int_{\mathbb{R}^{n}} u(y) \mathscr{N}(y, \varphi, D \backslash \Sigma) d y .
$$

Последняя формула другим способом доказана в [53].

ЗАмечАниЕ 16. Заметим, что всякое отображение $\varphi \in W_{q, \text { loc }}^{1}(D)$ при $q>n$ (гомеоморфизм $\left.\varphi \in W_{n, \text { lос }}^{1}(D)\right)$ обладает $\mathscr{N}$-свойством Лузина (см. [54-56]).

ОПРеДЕЛЕНИЕ 17. Пусть $\varphi: D \rightarrow D^{\prime}$ - гомеоморфизм евклидовых областей в $\mathbb{R}^{n}, n \geq 2$. Пример 12 показывает, что $D$ можно представить в виде объединения трех дизъюнктных борелевских множеств: $D=Z \cup \Sigma \cup(D \backslash(Z \cup \Sigma))$, где

1) $Z$ содержит множество $\left\{x \in D: J_{\varphi}(x)=0\right\}$ нулей объемной производной, и отличается от него на множество меры нуль: $\mathscr{H}^{n}\left(Z \backslash\left\{x \in D: J_{\varphi}(x)=0\right\}\right)=0$,

2) $\Sigma \subset D-$ множество сингулярности, т. е. множество, образ $\varphi(\Sigma)$ которого имеет положительную меру,

3) $D \backslash(Z \cup \Sigma)$ - множество, на котором отображение $\varphi$ обладает $\mathscr{N}$ свойством Лузина и во всех точках которого верно неравенство $0<J_{\varphi}(x)<\infty$.

Разложению области $D$ соответствует разложение в образе:

$$
D^{\prime}=\left(D^{\prime} \backslash\left(Z^{\prime} \cup \Sigma^{\prime}\right)\right) \cup Z^{\prime} \cup \Sigma^{\prime},
$$

где множества $Z^{\prime}=\varphi(\Sigma), \Sigma^{\prime}=\varphi(Z)$ играют ту же роль для обратного гомеоморфизма $\varphi^{-1}: D^{\prime} \rightarrow D$, что и множества $Z, \Sigma$ для прямого гомеоморфизма $\varphi$ (см. детали в $[12, \S 1])$. 
Кроме того, если $\varphi: D \rightarrow D^{\prime}$ принадлежит классу Соболева $W_{1, \mathrm{loc}}^{1}(D)$ (или $\varphi \in \mathrm{ACL}(D))$, то в $[12, \S 1]$ доказано, что

$$
\mathscr{H}^{n}\left(\left\{x \in D: J_{\varphi}(x)=0\right\} \Delta\{x \in D: \operatorname{det} D \varphi(x)=0\}\right)=0 .
$$

С учетом вышеизложенного с самого начала для отображений класса Соболева можно считать, что $Z \supset\{x \in D: \operatorname{det} D \varphi(x))=0\}, \mathscr{H}^{n}(Z \backslash\{x \in D: \operatorname{det} D \varphi(x)=$ $0\})=0$ и $\operatorname{det} D \varphi(x) \neq 0$ на множестве $D \backslash(Z \cup \Sigma)$.

Ниже будем применять введенные здесь обозначения.

1.1. От минимального набора конденсаторов к локализации функции искажения. В этом разделе покажем, что локализация функции искажения в теореме 3 может быть получена из набора только кубических конденсаторов. Другими словами, докажем, что если условие (2) выполняется только для кубических конденсаторов, то выполняются также и утверждения 1 и 3 теоремы 3. Этот подход контрастирует с классическими традициями в теории квазиконформных отображений, в которой в качестве минимального набора обычно рассматриваются сферические конденсаторы (см. [27]).

В следующей теореме в качестве системы открытых множеств $\mathscr{O}_{c}\left(D^{\prime}\right)$, на которых определена квазиаддитивная функция множества $\Psi$, рассматривается минимальная система открытых множеств в $D^{\prime}$ (см. определение 6), содержащая:

1) $D^{\prime}$

2) всякий открытый куб $Q$, если $\bar{Q} \subset D^{\prime}$;

$3)$ дополнение $Q_{2} \backslash \bar{Q}_{1}$, если $Q_{1}, Q_{2} \subset D^{\prime}$ - два куба с общим центром и $Q_{1} \subset Q_{2}$.

В качестве ограниченной квазиаддитивной функции множества рассматривается отображение $\Phi: \mathscr{O}_{c}\left(D^{\prime}\right) \rightarrow[0, \infty)$.

Теорема 18. Пусть заданы гомеоморфизм $\varphi: D \rightarrow D^{\prime}$ областей $D, D^{\prime} \subset$ $\mathbb{R}^{n}, n \geq 2$, и весовая локально суммируемая функция $\omega: D^{\prime} \rightarrow(0, \infty)$.

Если для любого кубического конденсатора $E=(\overline{Q(y, r)}, Q(y, R)) \subset D^{\prime} c$ прообразом $\varphi^{-1}(E)=\left(\varphi^{-1}(Q(y, r)), \varphi^{-1}(Q(y, R))\right)$ в $D$ выполняется неравенство

$$
\operatorname{cap}^{\frac{1}{q}}\left(\varphi^{-1}(E) ; L_{q}^{1}(D)\right) \leq \begin{cases}K_{p} \operatorname{cap}^{\frac{1}{p}}\left(E ; L_{p}^{1}\left(D^{\prime} ; \omega\right)\right), & 1<q=p<\infty, \\ \Psi_{q, p}^{\frac{1}{\sigma}}(U \backslash F) \operatorname{cap}^{\frac{1}{p}}\left(E ; L_{p}^{1}\left(D^{\prime} ; \omega\right)\right), & 1<q<p<\infty,\end{cases}
$$

где $\Psi_{q, p}$ - некоторая ограниченная квазиаддитивная функция множества, определенная на системе $\mathscr{O}_{c}\left(D^{\prime}\right)$, то верны следующие утверждения.

1. Гомеоморфизм $\varphi: D \rightarrow D^{\prime}$ принадлежит $W_{q, \mathrm{loc}}^{1}(D)$, имеет конечное искажение: $D \varphi(x)=0 \mathscr{H}^{n}$-п. в. на множестве $Z=\{x \in D \mid J(x, \varphi)=0\}$, и операторная функция искажения

$$
D \ni x \mapsto K_{q, p}^{1, \omega}(x, \varphi)= \begin{cases}\frac{|D \varphi(x)|}{|\operatorname{det} D \varphi(x)|^{\frac{1}{p}} \omega^{\frac{1}{p}}(\varphi(x))}, & \text { если } \operatorname{det} D \varphi(x) \neq 0, \\ 0, & \text { если } \operatorname{det} D \varphi(x)=0,\end{cases}
$$

принадлежит $L_{\sigma}(D)$, где $\sigma$ определяется соотношениями $\frac{1}{\sigma}=\frac{1}{q}-\frac{1}{p}$, если $1<$ $q<p<\infty$, и $\sigma=\infty$, если $1<q=p<\infty$; более того,

$$
K_{q, p}^{1, \omega}(x, \varphi) \leq \begin{cases}7^{\frac{n}{p}} n K_{p}^{p} & \text { при } 1<q=p<\infty, \\ 7^{\frac{n}{q}} n \Psi^{\prime}(\varphi(x))^{\frac{1}{\sigma}} & \text { при } 1<q<p<\infty\end{cases}
$$

для $\mathscr{H}^{n}$-п. в. $x \in D \backslash(Z \cup \Sigma)$. 
2. Оператор композиции $\varphi^{*}: L_{p}^{1}\left(D^{\prime} ; \omega\right) \cap \operatorname{Lip}_{l}\left(D^{\prime}\right) \rightarrow L_{q}^{1}(D), 1 \leq q \leq p<\infty$, ограничен; более того,

$$
\left\|\varphi^{*}\right\| \leq\left\|K_{q, p}^{1, \omega}(\cdot) \mid L_{\sigma}\left(\varphi^{-1}(A)\right)\right\| \leq \begin{cases}7^{\frac{n}{p}} n K_{p} & \text { в случае } 1<q=p<\infty, \\ 7^{\frac{n}{q}} n \Psi\left(D^{\prime}\right)^{\frac{1}{\sigma}} & \text { в случае } 1<q<p<\infty ;\end{cases}
$$

квазиаддитивная функция

$$
D^{\prime} \supset A \mapsto \widetilde{\Psi}_{q, p}(A)=\left\|K_{q, p}^{1, \omega}(\cdot) \mid L_{\sigma}\left(\varphi^{-1}(A)\right)\right\|^{\sigma}
$$

удовлетворяет следующим соотношениям:

(a) $\widetilde{\Psi}_{q, p}(U) \leq 7^{\frac{n \sigma}{q}} n^{\sigma} \Psi(U)$ для любого открытого множества $U \in \mathscr{O}_{c}\left(D^{\prime}\right)$,

(b) $\left\|\varphi_{A}^{*}\right\| \leq \Psi_{q, p}^{\frac{1}{\sigma}}(A)$, где $\left\|\varphi_{A}^{*}\right\|-$ норма оператора $\varphi_{A}^{*}: L_{p}^{1}(A ; \omega) \cap \operatorname{Lip}_{l}(A) \rightarrow$ $L_{q}^{1}\left(\varphi^{-1}(A)\right), A \subset D^{\prime}$ - открытое множество.

3. Для любого конденсатора $E=\left(F_{1}, F_{0}\right)$ в $D^{\prime}$ с прообразом $\varphi^{-1}(E)=$ $\left(\varphi^{-1}\left(F_{1}\right), \varphi^{-1}\left(F_{0}\right)\right)$ в $D^{\prime}$ выполняется неравенство

$$
\begin{aligned}
& \operatorname{cap}^{\frac{1}{q}}\left(\varphi^{-1}(E) ; L_{q}^{1}(D)\right) \\
& \leq \begin{cases}7^{\frac{n}{p}} n K_{p} \operatorname{cap}^{\frac{1}{p}}\left(E ; L_{p}^{1}\left(D^{\prime} ; \omega\right)\right), & 1<q=p<\infty, \\
7^{\frac{n}{q}} n \Psi_{q, p}^{\frac{1}{\sigma}}\left(D^{\prime} \backslash\left(F_{0} \cup F_{1}\right)\right) \operatorname{cap}^{\frac{1}{p}}\left(E ; L_{p}^{1}\left(D^{\prime} ; \omega\right)\right), & 1<q<p<\infty .\end{cases}
\end{aligned}
$$

4. Между классами гомеоморфизмов имеет место совпадение:

$$
Q \mathscr{R} Q_{q, p}\left(D^{\prime}, D ; \omega\right)=\mathscr{Q}_{q, p}\left(D^{\prime}, D ; \omega\right) .
$$

5. Утверждения теоремы 18 справедливы также и в случае $1=q \leq p<\infty$, $n=2$.

ДокАЗАТЕЛЬСТво. Фиксируем куб $Q(y, r) \Subset D^{\prime}$ и рассмотрим тестовую функцию $u(z)=\left(r-|z-y|_{\infty}\right)^{+}$. Эта функция, очевидно, удовлетворяет условиям леммы 2.3 из [12]. По ее заключению имеем $u \circ \varphi \in L_{q}^{1}\left(\varphi^{-1}(U)\right)$ и

$$
\begin{aligned}
& \left\|u \circ \varphi \mid L_{q}^{1}\left(\varphi^{-1}(Q(y, r))\right)\right\| \\
& \leq \begin{cases}K_{p}\left\|u \mid L_{p}^{1}(Q(y, r) ; \omega)\right\|=K_{p} \omega(Q(y, r))^{\frac{1}{p}}, & \text { если } q=p, \\
\Psi(Q(y, r))^{\frac{1}{\sigma}}\left\|u \mid L_{p}^{1}(Q(y, r) ; \omega)\right\|=\Psi(Q(y, r))^{\frac{1}{\sigma}} \omega(Q(y, r))^{\frac{1}{p}}, & \text { если } q<p,\end{cases}
\end{aligned}
$$

где $\omega(Q(y, r))=\int_{Q(y, r)} \omega(z) d z-$ весовая мера куба $Q(y, r)$.

Фиксируем произвольное натуральное число $1 \leq j \leq n$. Зададим в кубе $Q(y, r) n$-мерный открытый тетраэдр

$$
T_{j} Q(y, r)=\left\{z \in Q(y, r): y_{j}-r<z_{j}<y_{j},\left|z_{j}-y_{j}\right|>\max _{l \neq j}\left|z_{l}-y_{l}\right|\right\} .
$$

Заметим, что на прообразе $\varphi^{-1}\left(T_{j} Q(y, r)\right)$ композиция $u \circ \varphi$ равна $r+\varphi_{j}(x)-y_{j}$. Из (15) выводим, что $\varphi_{j} \in L_{q}^{1}\left(\varphi^{-1}\left(T_{j} Q(y, r)\right)\right)$.

Пусть $z=y-\frac{3}{4} e_{j}$, где $e_{j}-j$-й вектор стандартного базиса в $\mathbb{R}^{n}$. При таком выборе точки $z$ имеем включения

$$
Q(z, r / 4) \subset T_{j} Q(y, r) \subset Q(y, r) \subset Q(z, 7 r / 4) .
$$

С каждой точкой $z \in W$, где $W$ - произвольное компактно вложенное в $D^{\prime}$ открытое множество: $W \Subset D^{\prime}$, ассоциируем куб $Q(z, r / 4)$ такой, что $Q(z, 2 r) \Subset$ 
$D^{\prime}$. С учетом (15) имеем

$$
\begin{aligned}
& \left\|\nabla \varphi_{j}\left|L_{q}^{1}\left(\varphi^{-1}(Q(z, r / 4))\right)\|\leq\| u \circ \varphi\right| L_{q}^{1}\left(\varphi^{-1}(Q(y, r))\right)\right\| \\
& \leq\left\{\begin{array}{ll}
K_{p} \omega(Q(y, r))^{\frac{1}{p}} \\
\Psi(Q(y, r))^{\frac{1}{\sigma}} \omega(Q(y, r))^{\frac{1}{p}}
\end{array} \leq \begin{cases}K_{p} \omega(Q(z, 7 r / 4))^{\frac{1}{p}}, & \text { если } q=p, \\
\Psi(Q(z, 7 r / 4))^{\frac{1}{\sigma}} \omega(Q(z, 7 r / 4))^{\frac{1}{p}}, & \text { если } q<p,\end{cases} \right.
\end{aligned}
$$

$j=1, \ldots, n$. Так как любое компактное множество в $D^{\prime}$ может быть покрыто конечным набором кубов вида $Q(z, r / 4)$, из (16) выводим следующие свойства:

1) $\varphi_{j} \in L_{q, \mathrm{loc}}^{1}(D)$;

2) $\varphi \in L_{q, \mathrm{loc}}^{1}(D)$, так как число $1 \leq j \leq n$ произвольное.

Покажем, что

3) $\nabla \varphi_{j}(x)=0$ в $\mathscr{H}^{n}$-п. в. точках множества $\varphi^{-1}(E)$, где $E-$ множество нулевой меры в $D^{\prime}, 1 \leq j \leq n$.

Достаточно проверить свойство 3 для множества $E \subset D^{\prime}$ нулевой меры такого, что $\operatorname{diam} E<\infty$ и $\operatorname{dist}\left(E, \mathbb{R}^{n} \backslash D^{\prime}\right)>0$. Существует ограниченное открытое множество $V \Subset D^{\prime}$ такое, что $E \subset V$ и $\mathscr{H}^{n}(V)<\varepsilon$ для наперед заданного $\varepsilon>0$. Применяя теорему Безиковича [50] к открытому множеству $V$, по аналогии с [57] найдем счетный набор $\mathscr{W}=\left\{Q_{k}\right\}$ кубов $Q_{k}=Q_{k}\left(z_{k}, r_{k}\right)$ таких, что

(a) $\bigcup_{k=1}^{\infty} Q_{k}=V$

(b) для $Q_{k}=Q_{k}\left(z_{k}, r_{k}\right) \in \mathscr{W}$ верно условие $\left|z_{k}-\mathbb{R}^{n} \backslash V\right|_{\infty}=32 r_{k}$, где $|x-F|_{\infty}=\inf _{y \in F}|x-y|_{\infty}-$ расстояние от точки $x$ до множества $F \neq \varnothing ;$

(c) семейство $\mathscr{W}$ можно разбить на конечное число $N_{n}$ (зависящее только от размерности $n$ ) подсемейств таких, что внутри каждого из них кубы не пересекаются; аналогичное свойство справедливо и для семейства кубов $\mathscr{W}^{*}=$ $\left\{8 Q_{k}=Q_{k}\left(z_{k}, 8 r_{k}\right)\right\}$.

В соответствии с последним свойством последовательность $\left\{8 Q_{k}\right\}$ можно разбить на $N_{n}$ подсемейств $\left\{8 Q_{1 m}\right\}_{m=1}^{\infty}, \ldots,\left\{8 Q_{N_{n} m}\right\}_{m=1}^{\infty}$ так, что внутри каждого подсемейства кубы дизъюнктны: $8 Q_{k m} \cap 8 Q_{k l}=\varnothing$, если $m \neq l$, $k=1, \ldots, N_{n}$.

Применим последнее свойство для оценки левого интеграла в (16) при $q=p$ :

$$
\begin{aligned}
& \int_{\varphi^{-1}(V)}\left|\nabla \varphi_{j}(x)\right|^{p} d x \leq \sum_{k \in \mathbb{N}_{\varphi^{-1}}} \int_{\left.Q_{k}\left(z_{k}, r_{k}\right)\right)}\left|\nabla \varphi_{j}(x)\right|^{p} d x \\
& \leq K_{p}^{p} \sum_{k \in N_{n}} \sum_{m \in \mathbb{N}} \omega\left(8 Q_{k m}\right) \leq N_{n} K_{p}^{p} \omega(V) .
\end{aligned}
$$

Так как $\varepsilon-$ произвольное число, а интеграл Лебега суммируемой функции абсолютно непрерывен, свойство 3 в случае $q=p$ доказано.

Случай $q<p$ потребует применения неравенства Гёльдера: из (16) выводим

$$
\begin{aligned}
\int_{\varphi^{-1}(V)}\left|\nabla \varphi_{j}(x)\right|^{q} d x \leq \sum_{k \in \mathbb{N}_{\varphi^{-1}}} \int_{\left(Q_{k}\left(z_{k}, r_{k}\right)\right)}\left|\nabla \varphi_{j}(x)\right|^{q} d x \\
\leq \sum_{k \in N_{n}} \sum_{m \in \mathbb{N}} \Psi\left(8 Q_{k m}\right)^{\frac{q}{\sigma}} \omega\left(8 Q_{k m}\right)^{\frac{q}{p}} \leq \sum_{k \in N_{n}}\left(\sum_{m \in \mathbb{N}} \Psi\left(8 Q_{k m}\right)\right)^{\frac{q}{\sigma}}\left(\sum_{m \in \mathbb{N}} \omega\left(8 Q_{k m}\right)\right)^{\frac{q}{p}} \\
\leq \sum_{k \in N_{n}} \Psi\left(D^{\prime}\right)^{\frac{q}{\sigma}} \omega(V)^{\frac{q}{p}} \leq N_{n} \Psi\left(D^{\prime}\right)^{\frac{q}{\sigma}} \omega(V)^{\frac{q}{p}}
\end{aligned}
$$

По причине, описанной выше, свойство 3 в случае $q<p$ тоже доказано. 
4. Отображение $\varphi$ имеет конечное искажение: $D \varphi(x)=0$ в $\mathscr{H}^{n}$-п. в. точках множества $Z$ (поскольку по формуле (14) имеем $\mathscr{H}^{n}(\varphi(Z \backslash \Sigma))=0$, где $\Sigma \subset D$ - множество сингулярности отображения $\varphi$ меры нуль).

5. Из включений $Q(z, r) \subset T_{j} Q(y, 4 r) \subset Q(y, 4 r) \subset Q(z, 7 r)$ и оценки $(16)$ с помощью формулы замены переменной (14) и свойства 3 выводим соотношение

$$
\begin{aligned}
\int_{Q(z, r)} \frac{\left|\nabla \varphi_{j}\left(\varphi^{-1}(y)\right)\right|^{q}}{J\left(\varphi^{-1}(y), \varphi\right)} \chi_{D^{\prime} \backslash \varphi(\Sigma)}(y) d y \\
\quad \leq \begin{cases}K_{p}^{p} \omega(Q(z, 7 r)), & 1 \leq q=p<\infty, \\
\Psi(Q(z, 7 r))^{\frac{q}{\sigma}} \omega(Q(z, 7 r))^{\frac{q}{p}}, & 1 \leq q<p<\infty,\end{cases}
\end{aligned}
$$

где $J(x, \varphi)$ - якобиан отображения $\varphi$. Остается поделить обе части последнего неравенства на $\mathscr{H}^{n}(Q(z, 7 r))$, учесть равенство $\frac{q}{\sigma}+\frac{q}{p}=1$ и перейти к пределу при $r \rightarrow 0$ по предложению 11 и теореме Лебега о дифференцировании интеграла (см. пример 13). В пределе для $\mathscr{H}^{n}$-п. в. $z \in D^{\prime} \backslash \varphi(\Sigma)$ получаем поточечное соотношение

$$
\frac{\left|\nabla \varphi_{j}\left(\varphi^{-1}(z)\right)\right|}{J\left(\varphi^{-1}(z), \varphi\right)^{\frac{1}{q}} \omega(z)^{\frac{1}{p}}} \leq \begin{cases}7^{\frac{n}{p}} K_{p}^{p}, & 1 \leq q=p<\infty \\ 7^{\frac{n}{q}} \Psi^{\prime}(z)^{\frac{1}{\sigma}}, & 1 \leq q<p<\infty\end{cases}
$$

С учетом неравенства $|D \varphi(x)| \leq \sum_{j=1}^{n}\left|\nabla \varphi_{j}(x)\right|$ приходим к заключению, что при $q=p$ функция искажения $D \ni x \mapsto K_{p, p}^{1, \omega}(x, \varphi)$ (см. (3)) ограничена постоянной $7^{\frac{n}{p}} n K_{p}^{p}$, а при $q<p$ имеем неравенство

$$
\left(\frac{|D \varphi(x)|}{|\operatorname{det} D \varphi(x)|^{\frac{1}{q}} \omega^{\frac{1}{p}}(\varphi(x))}\right)^{\sigma}|\operatorname{det} D \varphi(x)| \leq 7^{\frac{n \sigma}{q}} n^{\sigma} \Psi^{\prime}(\varphi(x))|\operatorname{det} D \varphi(x)|
$$

для п. в. точек $x \in D \backslash(Z \cup \Sigma)$. Интегрируя левую и правую части полученного соотношения по множеству $D \backslash(Z \cup \Sigma)$ и заменяя переменную в правой части, по формуле (14) выводим

$$
\left\|K_{q, p}^{1, \omega}(\cdot) \mid L_{\sigma}(D)\right\| \leq 7^{\frac{n}{q}} n \Psi^{\frac{1}{\sigma}}\left(D^{\prime}\right) .
$$

Утверждение 1 теоремы 18 доказано.

Оценка (17) означает, что выполнены условия утверждения 3 теоремы 3. Следовательно, по теореме 3 справедливо утверждение 2 теоремы 18 . Импликация $2 \Rightarrow 3$ теоремы 18 - это импликация $1 \Rightarrow 2$ в теореме 3 (см. детали в [12]).

Из утверждения 3 теоремы 18 выводим утверждение 4.

Утверждение 5 теоремы 18 доказывается так же, как в теореме 9.

\section{§ 2. Непрерывные открытые дискретные отображения класса $\mathscr{Q}_{q, p}$, их свойства и аналитическое описание}

Наша ближайшая цель - получить свойства отображений $f \in S \mathscr{R} Q_{q, p}\left(D^{\prime}\right.$, $D ; \omega)$ или $f \in \mathscr{R} Q_{q, p}\left(D^{\prime}, D ; \omega\right)$, которые можно вывести непосредственно из определения 5 .

Для доказательства теорем этого параграфа понадобятся некоторые вспомогательные утверждения, которые докажем в следующем разделе.

2.1. Вспомогательные соотношения. Всякая локально суммируемая функция $\omega: D^{\prime} \rightarrow(0, \infty), D^{\prime} \subset \mathbb{R}^{n}$, задает весовую меру измеримого множества 
$A \subset D^{\prime}$ по правилу

$$
\omega(A)=\int_{A} \omega(y) d y .
$$

Лемма 19. При $1 \leq p<\infty$ для конденсатора $E=(F, U)$ в $D^{\prime}$ справедлива оценка сверху

$$
\operatorname{cap}\left(E ; L_{p}^{1}(U ; \omega)\right) \leq \frac{\omega(U \backslash F)}{\operatorname{dist}(F, \partial U)^{p}},
$$

где $\operatorname{dist}(F, \partial U)$ - евклидово расстояние между множеством $F$ и границей множества $U$.

ДокАзАТЕЛЬСтво. Положим $r=\operatorname{dist}(F, \partial U)$. В качестве допустимой функции для емкости сар $\left(E ; L_{p}^{1}(U ; \omega)\right)$ рассмотрим функцию

$$
u(y)=\max \left(0,1-\frac{\operatorname{dist}(y, F)}{r}\right) .
$$

Действительно, $u(y)=1$ в точках $y \in F, u(y)=0$ в точках $y \notin U$ и $|\nabla u(y)| \leq r^{-1}$ для п. в. $y \in U$. Следовательно,

$$
\operatorname{cap}\left(E ; L_{p}^{1}(U ; \omega)\right) \leq \int_{U \backslash F}|\nabla u(y)|^{p} \omega(y) d y \leq r^{-p} \omega(U \backslash F) .
$$

Лемма 20. Пусть $n-1<q<\infty$, если $n \geq 3$, или $1 \leq q<\infty$, если $n=2$. Для конденсатора $E=(F, U)$ в $D$ при условии связности множества $F$ имеет место неравенство

$$
\operatorname{cap}^{n-1}\left(E ; L_{q}^{1}(U)\right) \geq c_{1}^{n-1} \frac{(\operatorname{diam} F)^{q}}{\mathscr{H}^{n}(U)^{q-(n-1)}},
$$

где $c_{1}$ - постоянная в неравенстве Морри (см. ниже (19)), зависящая только от $n$ и $q$.

ДокАЗАтельство. В случае $n=2, q=1$ неравенство (18) является следствием свойства: 1-емкость любого конденсатора $E=(F, U) \subset \mathbb{R}^{2}$ равна cap $\left(E ; L_{1}^{1}(U)\right)=\inf _{\gamma} \mathscr{H}^{1}(\gamma)$, где $\mathscr{H}^{1}(\gamma)$ обозначает $\mathscr{H}^{1}$-меру (длину) гладкой замкнутой кривой $\gamma \subset U$, у которой ограниченная компонента связности дополнения $\mathbb{R}^{2} \backslash \gamma$ содержит $F$, и нижняя грань берется по всем таким кривым $\gamma$ [51]. Очевидно, что $\inf _{\gamma} \mathscr{H}^{1}(\gamma) \geq \operatorname{diam} F$.

Далее применим в модифицированной форме метод из [18, лемма 5]. Поскольку левая и правая части доказываемого неравенства инвариантны относительно движений и имеют одинаковую степень однородности относительно гомотетий, достаточно доказать лемму в том случае, когда $\operatorname{diam} F$ равен расстоянию между точками $0, T \in F$, причем точка $T$ находится на $x_{n}$-й оси: $T=(0,0, \ldots, 0,1)$. Таким образом, $\operatorname{diam} F=|T|=1$. Следовательно, любая плоскость $P_{A}$ размерности $n-1$, перпендикулярная $x_{n}$-й оси и проходящая через точку $A=\left(0,0, \ldots, 0, a_{n}\right), 0<a_{n}<1$, пересекает $F$ в некоторой точке $x_{A}$. Обозначим символом

$$
B_{A}=B\left(x_{A}, \operatorname{dist}\left(x_{A},\left(\mathbb{R}^{n} \backslash U\right) \cap P_{A}\right)\right)
$$

максимальный $(n-1)$-мерный шар с центром в точке $x_{A}$, находящийся в пересечении $U \cap P_{A}$.

Всякая функция $u \in L_{p}^{1}(U) \cap \operatorname{Lip}_{l}(U), u=1$ на $F$, носитель которой содержится в $U$, принимает на сфере

$$
S\left(x_{A}, \operatorname{dist}\left(x_{A},\left(\mathbb{R}^{n} \backslash U\right) \cap P_{A}\right)\right) \cap P_{A}
$$


значение 0 . Поэтому, записывая точку $x \in P_{A}$ в виде $x=\left(\xi, a_{n}\right)$, воспользуемся для $\mathscr{H}^{1}$-п. в. $a_{n} \in(0,1)$ неравенством Морри $[50$, разд. 4.5 .3$]$ в следующем виде:

$$
\int_{P_{A} \cap U}\left|\nabla u\left(\xi, a_{n}\right)\right|^{q} d \xi \geq c_{1} \mathscr{H}^{n-1}\left(B_{A}\right)^{1-\frac{q}{n-1}},
$$

где мера хаусдорфа $\mathscr{H}^{n-1}\left(B_{A}\right)$ совпадает с $(n-1)$-мерной Лебега шара $B_{A}$, а $c_{1}$ - постоянная, зависящая лишь от $n$ и $q$. Применяя ко второму интегралу в первой строки неравенство Гёльдера с показателями $\frac{q}{q-(n-1)}$ и $\frac{q}{n-1}$, выводим

$$
\begin{aligned}
(\operatorname{diam} F)^{q} & =\left(\int_{0}^{1} d a_{n}\right)^{q}=\left(\int_{0}^{1} \mathscr{H}^{n-1}\left(B_{A}\right)^{\frac{q-(n-1)}{q}} \cdot \mathscr{H}^{n-1}\left(B_{A}\right)^{\frac{n-1-q}{q}} d a_{n}\right)^{q} \\
& \leq\left(\int_{0}^{1} \mathscr{H}^{n-1}\left(B_{A}\right) d a_{n}\right)^{q-(n-1)}\left(\int_{0}^{1} \mathscr{H}^{n-1}\left(B_{A}\right)^{1-\frac{q}{n-1}} d a_{n}\right)^{n-1} \\
& \leq \frac{1}{c_{1}^{n-1}} \mathscr{H}^{n}(U)^{q-(n-1)}\left(\int_{U}|\nabla u(x)|^{q} d x\right)^{n-1} .
\end{aligned}
$$

В переходе от (20) к (21) для оценки сверху первого интеграла в (20) применяется формула Кавальери - Лебега. Отсюда получаем (18).

2.2. Свойства непрерывных открытых дискретных отображений класса $S \mathscr{R} Q_{q, p}$. В этом разделе основная цель - получить свойства отображений $f \in S \mathscr{R} Q_{q, p}\left(D^{\prime}, D ; \omega\right)$, которые можно вывести непосредственно из определения 5 .

Теорема 21. Пусть $n-1<q<\infty$, если $n \geq 3$, или $1 \leq q<\infty$, если $n=2$. Непрерывное открытое дискретное отображение $f: D^{\prime} \rightarrow D$ класса $S \mathscr{R} Q_{q, p}\left(D^{\prime}, D ; \omega\right)$ при $q \leq p<\infty$ обладает следующими свойствами:

1) отображение $f$ дифференцируемо $\mathscr{H}^{n}$-п. в. в области $D^{\prime}$;

2) $f$ имеет конечное искажение;

3) справедливы поточечная оценка

$$
|D f(y)| \leq c_{2} \begin{cases}K_{p}^{n-1} \cdot|\operatorname{det} D f(y)|^{\frac{p-(n-1)}{p}} \omega(y)^{\frac{n-1}{p}} & \text { при } q=p, \\ \left(\Psi_{q, p}^{\prime}(y)\right)^{\frac{n-1}{\sigma}}|\operatorname{det} D f(y)|^{\frac{q-(n-1)}{q}} \omega(y)^{\frac{n-1}{p}} & \text { при } q<p\end{cases}
$$

для $\mathscr{H}^{n}$-п. в. $y \in D^{\prime}$ с постоянной $c_{2}=2^{n} \alpha(n) c_{1}^{\frac{1-n}{q}}$, где $\alpha(n)=\mathscr{H}^{n}(B(0,1))=$ $\Gamma\left(\frac{1}{2}\right)^{n} / \Gamma\left(\frac{n}{2}+1\right)\left(\right.$ здесь $\left.\frac{1}{\sigma}=\frac{1}{q}-\frac{1}{p}\right)$, и для любого открытого множества $U \subset D^{\prime}$ вытекающие из нее соотношения

$$
\begin{aligned}
& \int_{U}|D f(y)| d y \leq c_{3} \cdot \begin{cases}K_{p}^{n-1} \cdot \mathscr{H}^{n}(f(U))^{\frac{p-(n-1)}{p}} \cdot \omega(U)^{\frac{n-1}{p}} & \text { при } q=p, \\
\Psi_{q, p}(U)^{\frac{n-1}{\sigma}} \cdot \mathscr{H}^{n}(f(U))^{\frac{q-(n-1)}{q}} \cdot \omega(U)^{\frac{n-1}{p}} & \text { при } q<p\end{cases} \\
& \text { с постоянной } c_{3}=c_{2} \cdot \mathscr{N}(f, U)^{\frac{q-(n-1)}{q}} .
\end{aligned}
$$

ДокАЗАтЕльство. І. На шаге 1 установим дифференцируемость отображения $f$. Воспользуемся схемой доказательства из [58] для случая $q=p=n$ ([59, лемма 1] при $n-1<q<p=n), \omega \equiv 1$ (другой способ доказательства получен в $\left[9\right.$, теорема 2]). С каждой точкой $y \in D^{\prime}$ свяжем сферический конденсатор $E_{r}=(\overline{B(y, r)}, B(y, 2 r))$, где $B(y, 2 r) \subset D^{\prime}$. Учитывая определение 
класса $\mathscr{Q}_{q, p}\left(D^{\prime}, D ; \omega\right)$ при $q<p$ и лемму 19 , получаем (для рассматриваемого класса отображений образ конденсатора тоже конденсатор)

$$
\begin{aligned}
\operatorname{cap}^{\frac{1}{q}}\left(f\left(E_{r}\right) ; L_{q}^{1}(f(B(y, 2 r)))\right) \leq \Psi_{q, p}(B(y, 2 r))^{\frac{1}{\sigma}} & \operatorname{cap}^{\frac{1}{p}}\left(E_{r} ; L_{p}^{1}(U ; \omega)\right) \\
& \leq \Psi_{q, p}(B(y, 2 r))^{\frac{1}{\sigma}} \frac{\omega(B(y, 2 r))^{\frac{1}{p}}}{r}
\end{aligned}
$$

(при $q=p$ вместо $\Psi_{q, p}(B(y, 2 r))^{\frac{1}{\sigma}}$ следует написать $K_{p}$ ).

Используя для оценки емкости слева лемму 20 :

выводим

$$
\operatorname{cap}^{\frac{1}{q}}\left(f\left(E_{r}\right) ; L_{q}^{1}(f(B(y, 2 r)))\right) \geq c_{1}^{\frac{1}{q}} \frac{(\operatorname{diam} f(\overline{B(y, r)}))^{\frac{1}{n-1}}}{\mathscr{H}^{n}(f(B(y, 2 r)))^{\frac{q-(n-1)}{q(n-1)}}},
$$

$$
\begin{aligned}
& \frac{\operatorname{diam} f(\overline{B(y, r)})}{r} \\
& \quad \leq \frac{2^{n} c_{1}^{\frac{1-n}{q}}}{(2 r)^{n}} \Psi_{q, p}(B(y, 2 r))^{\frac{n-1}{\sigma}} \mathscr{H}^{n}(f(B(y, 2 r)))^{\frac{q-(n-1)}{q}} \omega(B(y, 2 r))^{\frac{n-1}{p}} .
\end{aligned}
$$

Устремляя $r \rightarrow 0$, для $\mathscr{H}^{n}$-п. в. $y \in D^{\prime}$ имеем

$$
\varlimsup_{z \rightarrow y} \frac{|f(z)-f(y)|}{|z-y|} \leq 2^{n} \alpha(n) c_{1}^{\frac{1-n}{q}}\left(\Psi_{q, p}^{\prime}(y)\right)^{\frac{n-1}{\sigma}} \overline{\mathscr{V}}_{n}^{\prime}(y)^{\frac{q-(n-1)}{q}} \omega(y)^{\frac{n-1}{p}},
$$

где значения $\overline{\mathscr{V}}_{n}^{\prime}(y)$ и $\Psi_{q, p}^{\prime}(y)$, выраженные в (12) и предложении 11 , часть $\mathbf{I}$, конечны $\mathscr{H}^{n}$-п. в. в $D^{\prime}$. Поскольку правая часть $(24)$ конечна $\mathscr{H}^{n}$-п. в. в $D^{\prime}$, по теореме Степанова (см., например, $[49,50]$ ) отображение $f$ дифференцируемо $\mathscr{H}^{n}$-п. в. в $D^{\prime}$. Известно, что в точках дифференцируемости отображения $f$ левая часть в (24) равна $|D f(y)|$, а $\overline{\mathscr{V}}_{n}^{\prime}(y)=|\operatorname{det} D f(y)|$ (см., например, [49]).

В неравенствах (24), а также (26) и неравенстве в п. II доказательства (см. ниже) в случае $q=p$ вместо $\left(\Psi_{q, p}^{\prime}(\cdot)\right)^{\frac{n-1}{\sigma}}$ следует написать $K_{p}^{n-1}$.

II. С учетом вышеизложенного перепишем неравенство (24) следующим образом:

$$
|D f(y)| \leq c_{2}\left(\Psi_{q, p}^{\prime}(y)\right)^{\frac{n-1}{\sigma}}|\operatorname{det} D f(y)|^{\frac{q-(n-1)}{q}} \omega(y)^{\frac{n-1}{p}},
$$

где $c_{2}=2^{n} \alpha(n) c_{1}^{\frac{1-n}{q}}$. Отсюда выводим поточечную оценку (22). Далее, очевидно, верно следующее: $D f(y)=0 \mathscr{H}^{n}$-п. в. на множестве $Z^{\prime}=\left\{y \in D^{\prime}\right.$ : $\operatorname{det} D f(y)=0\}$ нулей якобиана $\operatorname{det} D f(y)$. Следовательно, $f$ имеет конечное искажение.

III. Для доказательства неравенства (23) следует проинтегрировать (22) и применить неравенство Гёльдера с учетом $\frac{p-(n-1)}{p}+\frac{n-1}{p}=1$ при $q=p$ или $\frac{n-1}{\sigma}+\frac{q-(n-1)}{q}+\frac{n-1}{p}=1$ при $q<p$ :

$\int_{U}|D f(y)| d y \leq c_{2}\left(\int_{U}|\operatorname{det} D f(y)| d y\right)^{\frac{q-(n-1)}{q}}\left(\int_{U} \Psi_{q, p}^{\prime}(y) d y\right)^{\frac{n-1}{\sigma}}\left(\int_{U} \omega(y) d y\right)^{\frac{n-1}{p}}$.

Поскольку $\int_{U}|\operatorname{det} D f(y)| d y \leq \mathscr{N}(f, U) \mathscr{H}^{n}(f(U))$ и $\int_{U} \Psi_{q, p}^{\prime}(y) d y \leq \Psi_{q, p}(U)$ (см. пример 12 и предложение 11), выводим (23).

Таким образом, $|D f(y)|$ локально суммируема в $D^{\prime}$. 
ЗАмЕЧАниЕ 22. В приведенном выше доказательстве вместо неравенств (9) используются более слабые соотношения

$$
\begin{cases}\operatorname{cap}^{\frac{1}{p}}\left(f\left(E_{r}\right) ; L_{p}^{1}(D)\right) \leq K_{p} A(r, \omega), & \text { если } q=p, \\ \operatorname{cap}^{\frac{1}{q}}\left(f\left(E_{r}\right) ; L_{q}^{1}(D)\right) \leq \Psi_{q, p}(B(y, 2 r) \backslash \overline{B(y, r)})^{\frac{1}{\sigma}} A(r, \omega), & \text { если } q<p,\end{cases}
$$
где

$$
A(r, \omega)=\left(\int_{B(y, 2 r)}|\nabla u(y)|^{p} \omega(y) d y\right)^{\frac{1}{p}}, \quad E_{r}=(\overline{B(y, r)}, B(y, 2 r)),
$$

a $u(y)=\max \left(0,1-\frac{\operatorname{dist}(y, \overline{B(y, r)})}{r}\right)$ - тестовая функция для оценки емкости $\operatorname{cap}^{\frac{1}{p}}\left(E_{r}\right)$ сверху (см. лемму 19).

2.3. Свойства регулярности непрерывных открытых дискретных отображений класса $\mathscr{R} Q_{q, p}$. В этом разделе продолжим исследование свойств регулярности отображений класса $\mathscr{R} Q_{q, p}$ : будут указаны условия, при выполнении которых отображение $f \in \mathscr{R} Q_{q, p}\left(D^{\prime}, D ; \omega\right)$ принадлежит классу Соболева. Заметим, что метод доказательства обобщает классический подход и неоднократно применялся многими авторами в частных случаях (см., например, безвесовой случай в [58] при $q=p=n$, [59] при $n-1<q<p=n$; весовой случай в [60] при $q=p=n=2$, [61] при $q=p=n$, [62] при $n-1<q=p<\infty$ и др.).

Теорема 23. Пусть $n-1<q<\infty$, если $n \geq 3$, или $1 \leq q<\infty$, если $n=2$. Всякое непрерывное открытое дискретное отображение $f: D^{\prime} \rightarrow D$ семейства $\mathscr{R} Q_{q, p}\left(D^{\prime}, D ; \omega\right), q \leq p<\infty$,

1) принадлежит классу Соболева $W_{1, \mathrm{loc}}^{1}\left(D^{\prime}\right)$;

2) имеет конечное искажение;

3) дифференцируемо $\mathscr{H}^{n}$-п. в. в области $D^{\prime}$;

4) удовлетворяет оценкам (22) и (23).

ДоказАтельство. Свойства 2-4 отображения $f \in \mathscr{Q}_{q, p}\left(D^{\prime}, D ; \omega\right)$ при $q \leq$ $p<\infty$, где $n-1<q<\infty$, если $n \geq 3$, или $1 \leq q<\infty$, если $n=2$, доказаны в теореме 21.

Остается доказать свойство 1. Проверим, что $f \in \operatorname{ACL}\left(D^{\prime}\right)$. C учетом локальной суммируемости частных производных (см. (24)) получим требуемое (см. эквивалентное описание функций $f \in W_{1, \mathrm{loc}}^{1}\left(D^{\prime}\right)$ в $[51, \S 1.1 .3$, теоремы 1, 2]).

Для доказательства $f \in \mathrm{ACL}\left(D^{\prime}\right)$ возьмем произвольный $n$-мерный открытый куб $P, P \Subset D^{\prime}$, с ребрами, параллельными координатным осям, и покажем, например, что $f$ абсолютно непрерывно на $\mathscr{H}^{n-1}$-п. в. сечениях куба $P$ прямыми, параллельными оси $x_{j}, j=1, \ldots, n$. Поскольку существует не более чем счетный набор кубов указанного выше вида, докажем абсолютную непрерывность отображения $f$ на пересечении $\mathscr{H}^{n-1}$-п. в. линий, параллельных оси $x_{j}$, с областью $D^{\prime}$. Так как $j=1, \ldots, n$ произвольно, теорема будет доказана.

Пусть $P_{j}$ - проекция $P$ на подпространство $y_{j}=0$, и $I-$ проекция $P$ на координатную ось $y_{j}$. Тогда $P=P_{j} \times I=\left\{\left(z, y_{j}\right): z \in P_{j}, y_{j} \in I\right\}$.

Пусть $\Psi_{q, p}$ - квазиаддитивная функция в определении 5 отображения $f \in$ $\mathscr{R} Q_{q, p}\left(D^{\prime}, D ; \omega\right)$. Она порождает ограниченную квазиаддитивную функцию $\Psi_{q, p}(A, P)$ открытого множества $A \subset \mathscr{O}\left(P_{j}\right), A \times I \in \mathscr{O}(P)$, по правилу: $\mathscr{O}\left(P_{j}\right) \ni$ $A \mapsto \Psi_{q, p}(A, P)=\Psi_{q, p}(A \times I)$ (см. определение 6$)$. В силу предложения 11 для 
$\mathscr{H}^{n-1}$-п. в. точек $z \in P_{j}$ (т. е. для всех точек $z \in P_{j} \backslash \Sigma_{0}$, где $\Sigma_{0} \subset P_{j}-$ некоторое множество нулевой $\mathscr{H}^{n-1}$-меры) существует конечная производная

$$
\Psi_{q, p}^{\prime}(z, P)=\lim _{r \rightarrow 0} \frac{\Psi_{q, p}\left(B_{j}(z, r), P\right)}{\mathscr{H}^{n-1}\left(B_{j}(z, r)\right)},
$$

где $\left.B_{j}(z, r)\right) \subset P_{j}$ обозначает $(n-1)$-мерный шар с центром в точке $z$ радиуса $r$.

Функция множества $\mathscr{V}_{n}: \mathscr{O}(P) \ni G \mapsto \mathscr{H}^{n}(f(G))$ является ограниченной монотонной $\varkappa$-квазиаддитивной функцией, определенной на открытых множествах $G \in \mathscr{O}(P)$, и порождает монотонную $\varkappa$-квазиаддитивную функцию

$$
\mathscr{O}\left(P_{j}\right) \ni A \mapsto \mathscr{V}_{n}(A, P)=\mathscr{V}_{n}(A \times I)=\mathscr{H}^{n}(f(A \times I))
$$

открытого множества $A \subset \mathscr{O}\left(P_{j}\right), A \times I \in \mathscr{O}(P)$, с постоянной $\varkappa=\mathscr{N}(f, P)$. По предложению $11 \overline{\mathscr{V}}_{n}^{\prime}(z, P)<\infty$ для $\mathscr{H}^{n-1}$-п. в. точек $z \in P_{j}$, так что неравенство $\overline{\mathscr{V}}_{n}^{\prime}(z, P)<\infty$ выполняется во всех точках $z \in P_{j} \backslash \Sigma^{\prime}$, где $\Sigma^{\prime} \subset P_{j}$ - некоторое множество нулевой $\mathscr{H}^{n-1}$-меры.

На сечении $I_{z}=\{z\} \times I$ куба $P$ возьмем произвольные дизъюнктные отрезки $\Delta_{1}, \Delta_{2}, \ldots, \Delta_{k}$ с длинами $b_{1}, b_{2}, \ldots, b_{k}$ соответственно, все концевые точки которых рациональны. Очевидно, совокупность всех таких отрезков счетнал.

Обозначим через $U_{i}$ открытое множество $\bigcup_{y \in \Delta_{i}} B(y, r)$. Пусть $r>0$ выбрано так, что открытые множества $U_{1}, U_{2}, \ldots, U_{k}$ дизъюнктны и $U_{i} \subset P, i=1, \ldots, k$.

Рассмотрим конденсаторы $E_{i}=\left(\Delta_{i}, U_{i}\right)$. Тогда по лемме 19

$$
\operatorname{cap}\left(E_{i} ; L_{p}^{1}\left(U_{i} ; \omega\right)\right) \leq \frac{\omega\left(U_{i}\right)}{r^{p}}=\frac{\int_{U_{i}} \omega(y) d y}{r^{p}}, \quad i=1, \ldots, k .
$$

С другой стороны, при $n-1<q<\infty$ из леммы 20 вытекает, что

$$
\operatorname{cap}^{\frac{n-1}{q}}\left(f\left(E_{i}\right) ; L_{q}^{1}\left(U_{i}\right)\right) \geq c_{1}^{\frac{n-1}{q}} \frac{\operatorname{diam} f\left(\Delta_{i}\right)}{\mathscr{H}^{n}\left(f\left(U_{i}\right)\right)^{\frac{q-(n-1)}{q}}} .
$$

Из этих двух неравенств, учитывая условие $f \in \mathscr{R} Q_{q, p}\left(D^{\prime}, \omega\right)$, выводим

$$
\operatorname{diam} f\left(\Delta_{i}\right) \leq \frac{c_{1}^{\frac{1-n}{q}}}{r^{n-1}} \mathscr{H}^{n}\left(f\left(U_{i}\right)\right)^{\frac{q-(n-1)}{q}}\left(\Psi_{q, p}\left(U_{i}\right)\right)^{\frac{n-1}{\sigma}} \omega\left(U_{i}\right)^{\frac{n-1}{p}} .
$$

Суммируя по $i=1, \ldots, k$, применяя неравенство Гёльдера и используя свойства квазиаддитивных функций, приходим к соотношениям

$$
\begin{aligned}
& \sum_{i=1}^{k} \operatorname{diam} f\left(\Delta_{i}\right) \\
& \quad \leq \frac{c_{1}^{\frac{1-n}{q}}}{r^{n-1}}\left(\sum_{i=1}^{k} \mathscr{H}^{n}\left(f\left(U_{i}\right)\right)\right)^{\frac{q-(n-1)}{q}}\left(\sum_{i=1}^{k} \Psi_{q, p}\left(U_{i}\right)\right)^{\frac{n-1}{\sigma}}\left(\sum_{i=1}^{k} \omega\left(U_{i}\right)\right)^{\frac{n-1}{p}} \\
& \leq c_{4}\left(\frac{\mathscr{V}_{n}\left(B_{j}(z, r), P\right)}{\mathscr{H}^{n-1}\left(B_{j}(z, r)\right)}\right)^{\frac{q-(n-1)}{q}}\left(\frac{\Psi_{q, p}\left(B_{j}(z, r), P\right)}{\mathscr{H}^{n-1}\left(B_{j}(z, r)\right)}\right)^{\frac{n-1}{\sigma}}\left(\frac{\sum_{i=1}^{k} \omega\left(U_{i}\right)}{\mathscr{H}^{n-1}\left(B_{j}(z, r)\right)}\right)_{(25)}^{\frac{n-1}{p}}
\end{aligned}
$$

где $c_{4}=2^{n-1} \alpha(n-1) c_{1}^{\frac{1-n}{q}} \cdot \mathscr{N}(f, P)^{\frac{q-(n-1)}{q}}$, a $\alpha(n-1)=\mathscr{H}^{n-1}\left(B_{j}(0,1)\right)$.

Устремляя $r$ к 0 , получаем следующее неравенство, справедливость которого при $\mathscr{H}^{n-1}$-п. в. $z \in P_{j}$ обусловлена существованием пределов в трех выражениях в скобках в соотношении (25) для $\mathscr{H}^{n-1}$-п. в. $z \in P_{j}$ (см. детали после 
формулы (26)):

$$
\sum_{i=1}^{k} \operatorname{diam} f\left(\Delta_{i}\right) \leq c_{4}\left(\overline{\mathscr{V}}_{n}^{\prime}(z, P)\right)^{\frac{q-(n-1)}{q}}\left(\Psi_{q, p}^{\prime}(z, P)\right)^{\frac{n-1}{\sigma}}\left(\int_{\bigcup_{i=1}^{k} \Delta_{i}} \omega\left(z, y_{n}\right) d y_{j}\right)^{\frac{n-1}{p}} .
$$

Первое частное в скобках (см. (25)) имеет конечный верхний предел во всех точках $z \in P_{j} \backslash \Sigma^{\prime}$. Второе частное имеет конечный предел во всех точках $z \in P_{j} \backslash \Sigma_{0}$ (см. предложение 11) (здесь $\mathscr{H}^{n-1}\left(\Sigma_{0}\right)=\mathscr{H}^{n-1}\left(\Sigma^{\prime}\right)=0$ ). Третье выражение в скобках (см. (25)) тоже имеет конечный предел для $\mathscr{H}^{n-1}$-п. в. точек $z \in P_{j}$. Чтобы в этом убедиться, рассмотрим отдельное слагаемое в третьем выражении в скобках в формуле (26), например, с номером $i$ (из существования предела для каждого слагаемого можно получить существование предела и для конечного набора слагаемых). Напомним, что $U_{i}=\bigcup_{y \in \Delta_{i}} B(y, r)$. По этой причине

$$
U_{i} \subset\left(B_{j}(z, r) \times \Delta_{i}\right) \cup\left(B_{j}(z, r) \times \alpha\right) \cup\left(B_{j}(z, r) \times \beta\right),
$$

где $\alpha, \beta$ в (27) суть промежутки на интервале $(\{z\} \times I) \cap U_{i}$ длины $r$, дополнительные к $\Delta_{i}$. Применяя теорему Фубини, запишем выбранное выше слагаемое в следующем виде:

$$
\begin{aligned}
& \frac{\omega\left(U_{i}\right)}{\mathscr{H}^{n-1}\left(B_{j}(z, r)\right)}=\frac{1}{\mathscr{H}^{n-1}\left(B_{j}(z, r)\right)} \int_{U_{i}} \omega(y) d y=\int_{\Delta_{i}} \omega\left(z, y_{j}\right) d y_{j} \\
& +\frac{1}{\mathscr{H}^{n-1}\left(B_{j}(z, r)\right)}\left(\int_{B_{j}(z, r)}\left(\int_{\Delta_{i}} \omega\left(w, y_{j}\right) d y_{j}-\int_{\Delta_{i}} \omega\left(z, y_{j}\right) d y_{j}\right) d w\right)+\mathfrak{R}(r)
\end{aligned}
$$

при $r \rightarrow 0$ (по теореме Фубини для всех точек $z \in P_{j} \backslash D_{j}$, где $\mathscr{H}^{n-1}\left(D_{j}\right)=0$, существует интеграл $\int_{z \times I} \omega\left(z, y_{j}\right) d y_{j}$, который в тех же точках $z \in P_{j}$ гарантирует конечность интеграла в правой части (28)). По теореме Лебега о дифференцировании интеграла первое выражение в строке (29) стремится ${ }^{6}$ к нулю для $\mathscr{H}^{n-1}$-п. в. $z \in P_{j}$, т. е. для всех $z \in P_{j} \backslash \Sigma_{i}$, за исключением некоторого множества $\Sigma_{i} \subset P_{j}$, имеющего $\mathscr{H}^{n-1}$-меру нуль. Остаточный член $\mathfrak{R}(r)$ в строке (29) неотрицательный и содержит два слагаемых, мажорируемых суммой

$$
\frac{1}{\mathscr{H}^{n-1}\left(B_{j}(z, r)\right)}\left(\int_{B_{j}(z, r)}\left(\int_{\alpha} \omega\left(w, y_{j}\right) d y_{j}+\int_{\beta} \omega\left(w, y_{j}\right) d y_{j}\right) d w\right),
$$

где $\alpha, \beta-$ промежутки на интервале $(\{z\} \times I) \cap U_{i}$ длины $r$, дополнительные к $\Delta_{i}$. Поскольку интегралы во внутренних круглых скобках стремятся к нулю при $r \rightarrow 0$, то $\mathfrak{R}(r)=o(1)$ при $r \rightarrow 0$.

С учетом того, что набор отрезков $\left\{\Delta_{i}\right\}$ не более чем счетный, неравенство (26) доказано для всех точек $z \in P_{j} \backslash\left(D_{j} \cup \Sigma^{\prime} \cup \bigcup_{i=0}^{\infty} \Sigma_{i}\right)$, где объединение $D_{j} \cup$

6) Здесь теорема Лебега о дифференцировании интеграла применяется в следующем виде:

$$
\lim _{r \rightarrow 0} \frac{1}{\mathscr{H}^{n-1}\left(B_{j}(z, r)\right)} \int_{B_{j}(z, r)}\left|\int_{\Delta_{i}} \omega\left(w, y_{j}\right) d y_{j}-\int_{\Delta_{i}} \omega\left(z, y_{j}\right) d y_{j}\right| d w=0
$$

для $\mathscr{H}^{n-1}$-п. в. точек $z \in P_{j}$. 
$\Sigma^{\prime} \cup \bigcup_{i=0}^{\infty} \Sigma_{i} \subset P_{j}$ имеет $\mathscr{H}^{n-1}$-меру нуль.

Неравенство (26) показывает также, что абсолютная непрерывность отображения $f:\{z\} \times I \rightarrow D$ при фиксированном $z$ обеспечена абсолютной непрерывностью интеграла $\int_{\{z\} \times I} \omega\left(z, y_{j}\right) d y_{j}$ на промежутке $I$. Следовательно, неравенство (26) можно распространить на произвольный конечный (а следовательно, и счетный) набор отрезков $\left\{\Delta_{i}\right\}$ (не обязательно с рациональными концевыми точками).

Так как $j$ может быть произвольным натуральным числом от 1 до $n$, абсолютная непрерывность отображения $f: D^{\prime} \rightarrow D$ доказана. Отсюда с учетом (23) доказано также, что $f \in W_{1, \mathrm{loc}}^{1}\left(D^{\prime}\right)$ (см. детали в [51]) $)^{7}$.

\section{§3. Новые примеры гомеоморфизмов класса $\mathscr{Q}_{q, p}$}

В этом параграфе добавим к теореме 9 новые примеры $\mathscr{Q}_{q, p}\left(D^{\prime}, D ; \omega\right)$-гомеоморфизмов и установим некоторые их новые свойства.

Пример 24. Пусть $\varphi: D \rightarrow D^{\prime}$ - гомеоморфизм класса Соболева $W_{p, \text { loc }}^{1}(D)$, $1<p<\infty$ при $n \geq 3$ и $1 \leq p<\infty$ при $n=2$, имеющий конечное искажение. Обратный гомеоморфизм $f=\varphi^{-1}: D^{\prime} \rightarrow D$ принадлежит классу $\mathscr{Q}_{p, p}\left(D^{\prime}, D ; \omega\right)$ с постоянной $K_{p}=1$ и с весовой функцией (30) (см. ниже).

Чтобы проверить справедливость примера 24 , докажем несколько свойств, представляющих независимый интерес. Во-первых, справедлива

Теорема 25. Пусть $\varphi: D \rightarrow D^{\prime}$ - гомеоморфизм класса Соболева $W_{p, \text { loc }}^{1}(D)$, $1 \leq p<\infty$, имеющий конечное искажение. Тогда весовая функция, определяемая соотношением

$$
D^{\prime} \ni y \mapsto \omega(y)= \begin{cases}\frac{\left|D \varphi\left(\varphi^{-1}(y)\right)\right|^{p}}{\left|\operatorname{det} D \varphi\left(\varphi^{-1}(y)\right)\right|}, & \text { если } y \in D^{\prime} \backslash\left(Z^{\prime} \cup \Sigma^{\prime}\right), \\ 1 & \text { иначе }\end{cases}
$$

локально суммируема: $\omega \in L_{1, \mathrm{loc}}\left(D^{\prime}\right)$, и оператор композиции

$$
\varphi^{*}: L_{p}^{1}\left(D^{\prime} ; \omega\right) \cap \operatorname{Lip}_{l}\left(D^{\prime}\right) \rightarrow L_{p}^{1}(D), \quad 1 \leq p<\infty,
$$

ограничен, причем $\left\|\varphi^{*}\right\| \leq\left\|K_{p, p}^{1, \omega}(\cdot) \mid L_{\infty}(D)\right\|=1$.

ДокАЗАтЕльство. Пусть $\varphi: D \rightarrow D^{\prime}$ - гомеоморфизм класса Соболева $W_{p, \text { loc }}^{1}(D), 1 \leq p<\infty$, имеющий конечное искажение. Исследуем условия на вес $\omega: D^{\prime} \rightarrow(0, \infty)$, гарантирующие ограниченность оператора $(31)$.

Пусть существует локально суммируемый вес $\omega: D^{\prime} \rightarrow(0, \infty)$ такой, что отображение $\varphi$ индуцирует ограниченный оператор композиции $\varphi^{*}: L_{p}^{1}\left(D^{\prime} ; \omega\right) \cap$ $\operatorname{Lip}_{l}\left(D^{\prime}\right) \rightarrow L_{p}^{1}(D), 1 \leq p<\infty$. Тогда для $\varphi: D \rightarrow D^{\prime}$ выполняются утверждения 1 и 3 теоремы 3 и, следовательно, операторная функция искажения (см. (3))

$$
D \ni x \mapsto K_{p, p}^{1, \omega}(x, \varphi)= \begin{cases}\frac{|D \varphi(x)|}{|\operatorname{det} D \varphi(x)|^{\frac{1}{p}} \omega^{\frac{1}{p}}(\varphi(x))}, & \text { если } \operatorname{det} D \varphi(x) \neq 0, \\ 0, & \text { если } \operatorname{det} D \varphi(x)=0,\end{cases}
$$

7) В монографии [51, $§ 1.1 .3$, теоремы 1,2$]$ доказано, что локально суммируемая функция $u: \Omega \rightarrow \mathbb{R}$ принадлежит классу Соболева $L_{p}^{1}(\Omega), p \geq 1$, тогда и только тогда, когда она может быть изменена на множестве нулевой $\mathscr{H}^{n}$-меры так, что измененная функция абсолютно непрерывна на $\mathscr{H}^{n-1}$-п. в. прямых, параллельных любой координатной оси, и ее обычные частные производные принадлежат $L_{p}(\Omega)$. При этом слабый градиент $\nabla u$ функции $u$ (в смысле теории обобщенных функций) совпадает $\mathscr{H}^{n}$-п. в. с обычным градиентом. 
принадлежит $L_{\infty}(D)$. Из (4) вытекает оценка нормы оператора композиции: $\left\|\varphi^{*}\right\| \leq\left\|K_{p, p}^{1, \omega}(\cdot) \mid L_{\infty}(D)\right\|$. Очевидно, условие $\left\|K_{p, p}^{1, \omega}(\cdot) \mid L_{\infty}(D)\right\|=1$ обеспечивает для оператора $\varphi^{*}$ оценку $\left\|\varphi^{*}\right\| \leq 1$. Другими словами, из приведенных соотношений выводим равенства $K_{p, p}^{1, \omega}(x, \varphi)=0$ на $Z$ и $K_{p, p}^{1, \omega}(x, \varphi)=1$ для $\mathscr{H}^{n}$ п. в. $x \in D \backslash Z$. Следовательно, на множестве $D^{\prime} \backslash\left(Z^{\prime} \cup \Sigma^{\prime}\right)$ в качестве веса можно взять измеримую функцию

$$
\omega(y)=\frac{\left|D \varphi\left(\varphi^{-1}(y)\right)\right|^{p}}{\left|\operatorname{det} D \varphi\left(\varphi^{-1}(y)\right)\right|},
$$

определенную для $\mathscr{H}^{n}$-п. в. $y \in D^{\prime} \backslash\left(Z^{\prime} \cup \Sigma^{\prime}\right)$. В силу формулы (14) имеем

$$
\begin{array}{r}
\int_{\varphi(W) \backslash\left(Z^{\prime} \cup \Sigma^{\prime}\right)} \omega(y) d y=\int_{\varphi(W) \backslash\left(Z^{\prime} \cup \Sigma^{\prime}\right)} \frac{\left|D \varphi\left(\varphi^{-1}(y)\right)\right|^{p}}{\left|\operatorname{det} D \varphi\left(\varphi^{-1}(y)\right)\right|} d y \\
=\int_{W \backslash(Z \cup \Sigma)}|D \varphi(x)|^{p} d x<\infty
\end{array}
$$

для компактно вложенной области $W \Subset D$. Заметим, что приведенные здесь соотношения не накладывают никаких условий на поведение веса (31) на множестве $Z^{\prime}=\varphi(\Sigma)$.

С учетом вышеизложенного определим вес в случае $\left|Z^{\prime}\right|>0$ по формуле $(30)$. При таком выборе вес $\omega: D^{\prime} \rightarrow(0, \infty)$ локально суммируем: $\omega \in$ $L_{1, \text { loc }}\left(D^{\prime}\right)$, а внешняя операторная функция искажения равна

$$
D \ni x \mapsto K_{p, p}^{1, \omega}(x, \varphi)= \begin{cases}1, & \text { если } \operatorname{det} D \varphi(x) \neq 0, \\ 0 & \text { иначе. }\end{cases}
$$

Следовательно, выполнены условие 3 и тем самым эквивалентное ему условие 1 теоремы 3. Кроме того, имеем оценку $\left\|\varphi^{*}\right\| \leq\left\|K_{p, p}^{1, \omega}(\cdot) \mid L_{\infty}(D)\right\|=1$ для оператора композиции.

В качестве следствия теорем 3 и 9 выводим

Следствие 26. Пусть $\varphi: D \rightarrow D^{\prime}-$ гомеоморфизм класса Соболева $W_{p, \text { loc }}^{1}(D), 1<p<\infty$ при $n \geq 3$ и $1 \leq p<\infty$ при $n=2$, имеющий конечное искажение. Тогда обратное отображение $f=\varphi^{-1}$ принадлежит семейству $\mathscr{Q}_{p, p}\left(D^{\prime}, D ; \omega\right)$.

ДокАЗАтЕльство. По теореме 9 отображение $f=\varphi^{-1}$ принадлежит класcу $\mathscr{Q}_{p, p}\left(D^{\prime}, D ; \omega\right)$ при $1<q$. Заметим, что доказательство импликации $1 \Rightarrow 2$ в теореме 3 , полученное в $[10,12]$ при $1<q$, работает также и при $n=2, q=1$.

Пример 24, теоремы 21, 23, 25 и следствие 26 приводят к следующему утверждению, в котором формулируются свойства регулярности гомеоморфизма, обратный к которому $\varphi: D \rightarrow D^{\prime}$ принадлежит классу Соболева $W_{p, \text { loc }}^{1}(D)$ и имеет конечное искажение.

Теорема 27. Пусть $\varphi: D \rightarrow D^{\prime}$ - гомеоморфизм класса Соболева $W_{p, \mathrm{loc}}^{1}(D)$, $n-1<p<\infty$ при $n \geq 3,1 \leq p<\infty$ при $n=2$, имеющий конечное искажение. Тогда обратный гомеоморфизм $f=\varphi^{-1}: D^{\prime} \rightarrow D$ обладает следующими свойствами:

1) принадлежит классу Соболева $W_{1, \mathrm{loc}}^{1}\left(D^{\prime}\right)$;

2) $f$ имеет конечное искажение;

3) гомеоморфизм $f$ дифференцируем $\mathscr{H}^{n}$-п. в. в области $D^{\prime}$; 
4) для $\mathscr{H}^{n}$-п. в. $y \in D^{\prime}$ справедлива оценка

$$
|D f(y)| \leq 2^{n} \alpha(n) c_{1}|\operatorname{det} D f(y)|^{\frac{p-(n-1)}{p}} \omega(y)^{\frac{n-1}{p}},
$$

а вместе с ней для любого открытого множества $U \subset D^{\prime}$ имеет место неравенство

$$
\int_{U}|D f(y)| d y \leq c_{2} \mathscr{H}^{n}(f(U))^{\frac{p-(n-1)}{p}} \omega(U)^{\frac{n-1}{p}}
$$

с весовой функцией (30) и постоянной $c_{2}=2^{n} \alpha(n) c_{1}$, где $\alpha(n)=\mathscr{H}^{n}(B(0,1))=$ $\Gamma\left(\frac{1}{2}\right)^{n} / \Gamma\left(\frac{n}{2}+1\right)$, а $c_{1}-$ постоянная в неравенстве $(20)$.

ДокАЗАтЕльство. Действительно, пример 24 и следствие 26 показывают, что в условиях теоремы 27 обратный гомеоморфизм $f=\varphi^{-1}: D^{\prime} \rightarrow D$ принадлежит классу $\mathscr{Q}_{p, p}\left(D^{\prime}, D ; \omega\right)$ с весовой функцией $(30)$ и постоянной $K_{p}=1$. Следовательно, по теореме 23 выполняются утверждения 1-4 теоремы.

ЗАмечАниЕ 28. Приведенное доказательство теоремы 27 новое, хотя отдельные ситуации были рассмотрены ранее. А именно, утверждения 1, 2 теоремы 27 доказаны в $[1$, теоремы $3.2,3.3]$ при $n=2, p=1$, и в работе $[2$, теорема 1.2 при $n \geq 3, p>n-1$, утверждение 3 теоремы 27 при $n \geq 3, p=n$ можно получить из монографии [6], в которой приведена также подробная библиография. Метод доказательства абсолютной непрерывности и дифференцируемости в теоремах 21 и 23, по существу, восходит к работе Д. Е. Меньшова [4] (см. изложение результатов Д. Е. Меньшова в [63]).

С помощью теоремы 27 покажем справедливость теоремы 3 в случае $1=$ $q \leq p<\infty, n=2$.

Следствие 29. Пусть гомеоморфизм $f: D^{\prime} \rightarrow D, D^{\prime}, D$ - области в $\mathbb{R}^{2}$, принадлежит семейству $\mathscr{Q}_{q, p}\left(D^{\prime}, D ; \omega\right), 1=q \leq p<\infty$. Тогда обратное отображение $\varphi=f^{-1}: D \rightarrow D^{\prime}$ обладает свойствами 1 и 3 теоремы 3.

ДоказАТЕЛЬСтво. В силу теоремы 23 гомеоморфизм $f: D^{\prime} \rightarrow D$ семейства $\mathscr{Q}_{q, p}\left(D^{\prime}, D ; \omega\right), 1 \leq q \leq p<\infty, n=2$, принадлежит классу Соболева $W_{1, \text { loc }}^{1}\left(D^{\prime}\right): f \in W_{1, \text { loc }}^{1}\left(D^{\prime}\right)$ и имеет конечное искажение. Следовательно, по теореме 27 , примененной к гомеоморфизму $f: D^{\prime} \rightarrow D$ класса $W_{1, \mathrm{loc}}^{1}\left(D^{\prime}\right)$ с конечным искажением (см. определение в (1)), обратное отображение $\varphi=f^{-1}: D \rightarrow$ $D^{\prime}$ принадлежит классу Соболева $W_{1, \text { loc }}^{1}(D): \varphi \in W_{1, \text { loc }}^{1}(D)$ и имеет конечное искажение. Далее к отображению $\varphi \in W_{1, \mathrm{loc}}^{1}(D)$ следует применить рассуждения из [12, лемма 2.5 и разд. 2.5], на основании которых выводим ${ }^{8)}$, что для отображения $\varphi: D \rightarrow D^{\prime}$ справедливы утверждения 1 и 3 теоремы 3.

ПримеР 30. Пусть $n-1<s<\infty$, а $f: D^{\prime} \rightarrow D$ - гомеоморфизм открытых областей $D^{\prime}, D \subset \mathbb{R}^{n}, n \geq 2$, такой, что

1) $f \in W_{n-1, \mathrm{loc}}^{1}\left(D^{\prime}\right)$;

2) отображение $f$ имеет конечное искажение: $D f(y)=0 \mathscr{H}^{n}$-п. в. на множестве $Z=\left\{y \in D^{\prime} \mid \operatorname{det} D f(y)=0\right\}$;

$3)$ внешняя операторная функция искажения

$$
D^{\prime} \ni y \mapsto K_{n-1, s}^{1,1}(y, f)= \begin{cases}\frac{|D f(y)|}{|\operatorname{det} D f(y)|^{\frac{1}{s}}}, & \text { если } \operatorname{det} D f(y) \neq 0, \\ 0, & \text { если } \operatorname{det} D f(y)=0,\end{cases}
$$

8) Рассуждения, содержащиеся в упомянутых фрагментах из [12], применимы для всякого гомеоморфизма $\varphi: D \rightarrow D^{\prime}$ класса Соболева $W_{1, \text { loc }}^{1}(D)$, имеющего конечное искажение и удовлетворяющего соотношениям (2). 
принадлежит $L_{\sigma}(D)$, где $\sigma=(n-1) p, p=\frac{s}{s-(n-1)}$.

Тогда обратный гомеоморфизм $\varphi=f^{-1}: D \rightarrow D^{\prime}$ обладает свойствами

4) $\varphi \in W_{p, \text { loc }}^{1}(D), p=\frac{s}{s-(n-1)}$

5) $\varphi$ имеет конечное искажение, а прямой гомеоморфизм $f: D^{\prime} \rightarrow D$

6 п) принадлежит классу $\mathscr{Q}_{p, p}\left(D^{\prime}, D ; \omega\right)$ с постоянной $K_{p}=1$ и с весовой функцией $\omega \in L_{1, \mathrm{loc}}\left(D^{\prime}\right)$, определяемой по формуле

$$
\omega(y)= \begin{cases}\frac{|\operatorname{adj} D f(y)|^{p}}{|\operatorname{det} D f(y)|^{p-1}}, & \text { если } y \in D^{\prime} \backslash Z^{\prime}, \\ 1 & \text { иначе }\end{cases}
$$

где $Z^{\prime}=\left\{y \in D^{\prime}: D f(y)=0\right\}$.

ДоказАтеЛЬСтво. Известно (см. [20, теорема 4]), что если гомеоморфизм $f: D^{\prime} \rightarrow D$ удовлетворяет сформулированным условиям, то обратный гомеоморфизм $\varphi=f^{-1}: D \rightarrow D^{\prime}$ обладает свойствами

4) $\varphi \in W_{p, \mathrm{loc}}^{1}(D), p=\frac{s}{s-(n-1)}$;

5) $\varphi$ имеет конечное искажение.

По условию (35) функция $K_{n-1, s}^{1,1}(y, f), n-1<s<\infty$, принадлежит $L_{\sigma}\left(D^{\prime}\right)$, $\sigma=(n-1) p, p=\frac{s}{s-(n-1)}$. Следовательно, функция $K_{n-1, s}^{1,1}(y, f)^{\sigma}$ суммируема на $D^{\prime} \backslash Z^{\prime}$. Далее непосредственно проверяем, что $\frac{(n-1) p}{s}=p-1$, поэтому с учетом известного неравенства $|D f(y)|^{n-1} \geq|\operatorname{adj} D f(y)|^{s}$ получаем

$$
K_{n-1, s}^{1,1}(y, f)^{\sigma}=\frac{\left(|D f(y)|^{n-1}\right)^{p}}{|\operatorname{det} D f(y)|^{\frac{(n-1) p}{s}}} \geq \frac{|\operatorname{adj} D f(y)|^{p}}{|\operatorname{det} D f(y)|^{p-1}} \quad \text { при } y \in D^{\prime} \backslash Z^{\prime} .
$$

Из суммируемости левой части соотношений (37) выводим суммируемость на множестве $D^{\prime} \backslash Z^{\prime}$ правой части (37). Следовательно, весовая функция (36) локально суммируема.

Заметим, что для $\mathscr{H}^{n}$-п. в. $y \in D^{\prime} \backslash Z^{\prime}$ справедливо равенство

$$
\frac{|D \varphi(f(y))|^{p}}{|\operatorname{det} D \varphi(f(y))|}=\frac{|\operatorname{adj} D f(y)|^{p}}{|\operatorname{det} D f(y)|^{p-1}} .
$$

Поэтому функция искажения

$$
D \ni x \mapsto K_{p, p}^{1, \omega}(x, \varphi)= \begin{cases}\frac{|D \varphi(x)|}{|\operatorname{det} D \varphi(x)|^{\frac{1}{p}} \omega^{\frac{1}{p}}(\varphi(x))}, & \text { если } \operatorname{det} D \varphi(x) \neq 0, \\ 0, & \text { если } \operatorname{det} D \varphi(x)=0,\end{cases}
$$

равна единице $\mathscr{H}^{n}$-п. в. на множестве $\{x \in D: \operatorname{det} D \varphi(x) \neq 0\}$.

$\mathrm{C}$ помощью теорем 3 и 9 выводим, что гомеоморфизм $\varphi: D \rightarrow D^{\prime}$ имеет обратный $f=\varphi^{-1}: D^{\prime} \rightarrow D$, принадлежащий классу $\mathscr{Q}_{p, p}\left(D^{\prime}, D ; \omega\right)$ с весовой функцией (36).

ЗАмЕчАниЕ 31. Сформулированные в примере 30 и следствиях 33 и 34 (см. ниже) свойства гомеоморфизма $f$ и обратного к нему новые. Близкий к примеру 30 результат при $s=p=n$ и весовой функцией $\omega(y)=K_{n-1, n}^{1,1}(y, f)^{(n-1) n}=$ $\left(\frac{|D f(y)|^{n}}{|\operatorname{det} D f(y)|}\right)^{n-1}$ в точках $y \in D^{\prime} \backslash Z^{\prime}$ вместо правой части (38) сформулирован в [64] на языке модулей семейств кривых.

ПримеР 32. Пусть $n-1<s<\infty$, а $f: D^{\prime} \rightarrow D$ - гомеоморфизм открытых областей $D^{\prime}, D \subset \mathbb{R}^{n}, n \geq 2$, такой, что

1) $f \in W_{n-1, \mathrm{loc}}^{1}\left(D^{\prime}\right)$;

2) отображение $f$ имеет конечное коискажение: $\operatorname{adj} D f(y)=0 \mathscr{H}^{n}$-п. в. на множестве $Z=\left\{y \in D^{\prime} \mid \operatorname{det} D f(y)=0\right\}$; 
3) внутренняя операторная функция искажения

$$
D^{\prime} \ni y \mapsto \mathscr{K}_{n-1, s}^{1,1}(y, f)= \begin{cases}\frac{|\operatorname{adj} D f(y)|}{|\operatorname{det} D f(y)|^{\frac{n-1}{s}}}, & \text { если } \operatorname{det} D f(y) \neq 0, \\ 0, & \text { если } \operatorname{det} D f(y)=0,\end{cases}
$$

принадлежит $L_{p}\left(D^{\prime}\right)$, где $p=\frac{s}{s-(n-1)}, n-1<s<\infty$.

Тогда обратный гомеоморфизм $\varphi=f^{-1}: D \rightarrow D^{\prime}$ обладает свойствами

4) $\varphi \in W_{p, \mathrm{loc}}^{1}(D), p=\frac{s}{s-(n-1)}$;

5) $\varphi$ имеет конечное искажение, а прямой гомеоморфизм $f: D^{\prime} \rightarrow D$

$6)$ принадлежит классу $\mathscr{Q}_{p, p}\left(D^{\prime}, D ; \omega\right)$ с постоянной $K_{p}=1$ и с весовой функцией (36);

7) имеет конечное искажение при $n-1<s<n+\frac{1}{n-2}$.

ДокАЗАтЕЛЬСтво. Известно (см. [20, теорема 3]), что если гомеоморфизм $f: D^{\prime} \rightarrow D$ удовлетворяет сформулированным условиям, то обратный гомеоморфизм $\varphi=f^{-1}: D \rightarrow D^{\prime}$ обладает свойствами

4) $\varphi \in W_{p, \text { loc }}^{1}(D)$,

5) $\varphi$ имеет конечное искажение.

Заметим, что по условию (40) функция $\mathscr{K}_{n-1, s}^{1,1}(y, f)$ принадлежит $L_{p}\left(D^{\prime}\right)$, $n-1<s<\infty$. Следовательно, функция $\mathscr{K}_{n-1, s}^{1,1}(y, f)^{p}$ суммируема на $D^{\prime} \backslash Z^{\prime}$ Далее непосредственно проверяем, что

$$
\mathscr{K}_{n-1, s}^{1,1}(y, f)^{p}=\left(\frac{|\operatorname{adj} D f(y)|}{|\operatorname{det} D f(y)|^{\frac{n-1}{s}}}\right)^{p}=\frac{|\operatorname{adj} D f(y)|^{p}}{|\operatorname{det} D f(y)|^{p-1}} \quad \text { при } y \in D^{\prime} \backslash Z^{\prime} .
$$

Следовательно, хотя предпосылки в примере 32 отличны от таковых в примере 30, приходим к той же самой весовой функции (36), которая локально суммируема в силу (41) и условия 3 примера 32.

Заметим, что для $\mathscr{H}^{n}$-П. в. $y \in D^{\prime} \backslash Z^{\prime}$ справедливо равенство (38). Поэтому функция искажения $D \ni x \mapsto K_{p, p}^{1, \omega}(x, \varphi)$, определенная в (39), равна единице $\mathscr{H}^{n}$-п. в. на множестве $\{x \in D: \operatorname{det} D \varphi(x) \neq 0\}$. Тем самым функция искажения $D \ni x \mapsto K_{p, p}^{1, \omega}(x, \varphi)$ определена корректно и $\left\|K_{p, p}^{1, \omega}(\cdot, \varphi)\right\|=1$.

По утверждению 1 теоремы 3 отображение $\varphi: D \rightarrow D^{\prime}$ порождает ограниченный оператор композиции $\varphi^{*}: L_{p}^{1}\left(D^{\prime} ; \omega\right) \cap \operatorname{Lip}_{l}\left(D^{\prime}\right) \rightarrow L_{q}^{1}(D)$. Отсюда выводим, что отображение $f=\varphi^{-1}: D^{\prime} \rightarrow D$ принадлежит классу $\mathscr{Q}_{p, p}\left(D^{\prime}, D ; \omega\right)$ с весовой функцией (36).

Из соотношения $n-1<s<n+\frac{1}{n-2}$ имеем $n-1<p$. Следовательно, по теореме 27 гомеоморфизм $f: D^{\prime} \rightarrow D$ имеет конечное искажение.

Утверждения 6 и 7 также доказаны.

Следствие 33. Всякий гомеоморфизм $f: D^{\prime} \rightarrow D$ в примерах 30 и 32 обладает дополнительными свойствами: в $D^{\prime}$;

1) неравенство (9) справедливо для произвольного конденсатора $E=\left(F_{1}, F_{0}\right)$

2) при $n-1<s<n+\frac{1}{n-2}$ гомеоморфизм $f: D^{\prime} \rightarrow D$ дифференцируем $\mathscr{H}^{n}$-П. в. в $D^{\prime}$.

ДоказАтеЛьство. Утверждение 1 вытекает из замечания 10 и неравенства (4), а утверждение 2 - из теоремы 27 с учетом того, что соотношения $n-1<s<n+\frac{1}{n-2}$ обеспечивают неравенство $n-1<p$. 
Следствие 34. Пусть гомеоморфизм $f: D \rightarrow D^{\prime}$ обладает свойствами 1-3 примеров 30 и 32 . Тогда

1) при условии $n-1<s<n+\frac{1}{n-2}$ ограничение гомеоморфизма $f: U^{\prime} \rightarrow$ $U$, где $U^{\prime} \Subset D^{\prime}-$ компактно вложенная область, а $U=f\left(U^{\prime}\right)$, индуцирует ограниченный оператор композиции

$$
f^{*}: L_{\frac{p}{p-(n-1)}}^{1}(U) \rightarrow L_{1}^{1}\left(U^{\prime}\right)
$$

2) при условии $n \leq s<n+\frac{1}{n-2}$ гомеоморфизм $f$ обладает $\mathscr{N}^{-1}$-свойством Лузина;

3) при условии $n \leq s<n+\frac{1}{n-2}$ гомеоморфизм $f$ имеет якобиан в $D^{\prime}$, отличный от нуля $\mathscr{H}^{n}$-п. в.

ДоказАтельство. 1. Рассмотрим компактно вложенную область $U^{\prime} \Subset D^{\prime}$ и произвольную функцию $u \in \operatorname{Lip}(U)$, где $U=f\left(U^{\prime}\right)$.

Из условия $n-1<s<n+\frac{1}{n-2}$ имеем неравенство $n-1<p<\infty$ для параметра $p$. Следовательно, в силу свойств, сформулированных в примерах 30 и 32 , обратный гомеоморфизм $\varphi=f^{-1}: D \rightarrow D^{\prime}$ удовлетворяет условиям теоремы 27. Применяя неравенство (33) в последующих оценках, получаем соотношения

$$
\begin{aligned}
& \int_{U^{\prime}}|\nabla(u \circ f)(y)| d y \leq \int_{U^{\prime}}|\nabla u|(f(y))|D f(y)| d y \\
& \leq c_{2} \int_{U^{\prime}}|\nabla u|(f(y))|\operatorname{det} D f(y)|^{\frac{p-(n-1)}{p}} \omega(y)^{\frac{n-1}{p}} d y \\
& \leq c_{2}\left(\int_{U^{\prime}}(|\nabla u|(f(y)))^{\frac{p}{p-(n-1)}}|\operatorname{det} D f(y)| d y\right)^{\frac{p-(n-1)}{p}}\left(\int_{U^{\prime}} \omega(y) d y\right)^{\frac{n-1}{p}} \\
& \leq c_{2}\left(\int_{U^{\prime}} \omega(y) d y\right)^{\frac{n-1}{p}}\left(\int_{U}|\nabla u(x)|^{\frac{p}{p-(n-1)}} d x\right)^{\frac{p-(n-1)}{p}},
\end{aligned}
$$

где так же, как и в (34), принято сокращение $c_{2}=2^{n} \alpha(n) c_{1}$. Неравенство между (43) и (44) означает, что отображение $f: U^{\prime} \rightarrow U$ индуцирует ограниченный оператор композиции (42).

2. Заметим, что из условия $n \leq s<n+\frac{1}{n-2}$ имеем условие $n-1<p \leq n$ для параметра $p$. В силу $\left[19\right.$, теорема 4] гомеоморфизм $f$ обладает $\mathscr{N}^{-1}$-свойством Лузина. Для удобства читателей сформулируем цитируемую здесь теорему.

Предложение 35 [19, теорема 4]. Если измеримое отображение $\varphi: D \rightarrow$ $D^{\prime}$ порождает ограниченный оператор композиции

$$
\varphi^{*}: L_{p}^{1}\left(D^{\prime}\right) \cap \operatorname{Lip}_{l}\left(D^{\prime}\right) \rightarrow L_{q}^{1}(D), \quad 1 \leq q \leq p \leq n,
$$

то $f$ обладает $\mathscr{N}^{-1}$-свойством Лузина.

3. Из [19, следствие 4] выводим, что якобиан гомеоморфизма $f$ отличен от нуля $\mathscr{H}^{n}$-п. в. в $U^{\prime}$ (это свойство выводится из свойства 2 следствия с помощью формулы замены переменной (13)). Так как $U^{\prime} \subset D^{\prime}-$ произвольная область, следствие доказано.

ЗАмечание 36. Сформулированные в примере 32 и следствиях 33 и 34 свойства гомеоморфизма $f$ и обратного к нему новые, за исключением дифференцируемости гомеоморфизма $f$ в примере 32 при $s=n, n \geq 3$ (случай $s=n$ рассмотрен в [65]). 


\section{$\S 4$. О регулярности гомеоморфизмов на группе Карно, обратных к соболевским}

В этом параграфе приведем обобщения утверждений 1 и 2 этой работы для гомеоморфизмов групп Карно. Метод доказательства ACL-свойства квазиконформных отображений на группах Гейзенберга разработан в работах $[66,67]$ и существенно отличается в деталях от классического метода, известного в евклидовых пространствах. Этот метод был применен позднее для доказательства ACL-свойства более сложных аналитических объектов (см., например, [18] и др.).

Ниже адаптируем новые доказательства утверждений 1 и 2 для демонстрации справедливости их обобщений на группах Карно. По существу, показываем, что на группах Карно можно успешно применить рассуждения, восходящие к классической работе Д. Е. Меньшова [68].

\section{1. Определение основных структур на группах Карно.}

4.1.1.Группой Карно [69-72] называется связная односвязная нильпотентная группа Ли $\mathbb{G}$, алгебра Ли $\mathscr{G}$ которой разлагается в прямую сумму $V_{1} \oplus \cdots \oplus$ $V_{m}$ векторных пространств таких, что $\left[V_{1}, V_{k}\right]=V_{k+1}$ для $1 \leq k \leq m-1$ и $\left[V_{1}, V_{m}\right]=\{0\}, \operatorname{dim} V_{1} \geq 2$. Далее применяем обозначения $x \cdot y$ для произведения двух элементов $x, y$ группы $\mathbb{G}$ и $e$ для нейтрального элемента группы. Подпространство $V_{1} \subset \mathscr{G}$ называется горизонтальным.

4.1.2. АЛГЕБРА ЛИ ГРУППЫ КАРНО И ЭКСПОНЕНЦИАЛЬНОЕ ОТОБРАЖЕниЕ. Пусть $X_{11}, \ldots, X_{1 n_{1}}$ - левоинвариантные векторные поля, образующие базис пространства $V_{1}$. Поскольку они порождают $\mathscr{G}$, для каждого $i, 1<i \leq m$, можно выбрать базис $X_{i j}, 1 \leq j \leq n_{i}=\operatorname{dim} V_{i}$ в $V_{i}$, образованный коммутаторами базисных полей $X_{1 k} \in V_{1}$ порядка $i-1$. Так как алгебра $\mathscr{G}$ нильпотентная, каждый элемент $x \in \mathbb{G}$ можно идентифицировать с точками пространства $\mathbb{R}^{n_{1}+\cdots+n_{m}}$ посредством экспоненциального отображения:

$$
x=\exp \left(\sum_{\substack{1 \leq i \leq m, 1 \leq j \leq n_{i}}} x_{i j} X_{i j}\right) .
$$

Диффеоморфизм ехр : $\mathscr{G} \rightarrow \mathbb{G}$ - это глобальная система координат: каждому элементу $x \in \mathbb{G}$ соответствует единственный набор чисел $\left\{x_{i j}\right\} \in \mathbb{R}^{N}, N=n_{1}+$ $\cdots+n_{m}$. Более того, удобно отождествить элементы $\mathscr{G}$ с точками пространства $\mathbb{R}^{N}$ так, чтобы экспоненциальное отображение $\exp : \mathscr{G} \rightarrow \mathbb{G}$ было тождественно [71]. Последнее означает, что элементы алгебры $\mathscr{G}$ и группы $\mathbb{G}$ - это одни и те же точки в $\mathbb{R}^{N}$, над которыми совершаются операции, зависящие от выбора структуры. При таком выборе системы координат единица $е$ группы - это 0 , а обратный $x^{-1}$ к элементу $x \in \mathbb{G}-$ это $-x$.

Растяжения $\delta_{t}$, определенные как $x \mapsto \delta_{t} x=\left(t^{i} x_{i j}\right)_{1 \leq i \leq m, 1 \leq j \leq n_{i}}$, суть автоморфизмы как алгебры $\mathscr{G}$, так и группы $\mathbb{G}$ для каждого $t>0$.

4.1.3. ПримеРы. Евклидово пространство $\mathbb{R}^{n}$ с его стандартной структурой является примером абелевой группы: векторные поля $\frac{\partial}{\partial x_{i}}, i=1, \ldots, n$, не имеют нетривиальных коммутационных соотношений и составляют базис соответствующей алгебры Ли.

Группа Гейзенберга $\mathbb{H}^{n}$ является примером неабелевой группы Карно. Алгебра Ли этой группы имеет размерность $2 n+1$. Векторные поля

$$
X_{i}=\frac{\partial}{\partial x_{i}}+2 y_{i} \frac{\partial}{\partial t}, \quad Y_{i}=\frac{\partial}{\partial y_{i}}-2 x_{i} \frac{\partial}{\partial t}, \quad T=\frac{\partial}{\partial t}, \quad i=1, \ldots, n,
$$


образуют базис алгебры Гейзенберга (здесь группа Гейзенберга $\mathbb{H}^{n}$ отождествлена с пространством $\left.\mathbb{R}^{2 n+1}=\left\{(x, y, t): x, y, \in \mathbb{R}^{n}, t \in \mathbb{R}\right\}\right)$. Единственные нетривиальные коммутационные соотношения суть $\left[X_{i}, Y_{i}\right]=-4 T, i=1, \ldots, n$. Таким образом, $V=V_{1} \oplus V_{2}$, где $V_{1}=\operatorname{span}\left\{X_{1}, \ldots, X_{n}, Y_{1}, \ldots, Y_{n}\right\}$ и $V_{2}=\operatorname{span}\{T\}-$ одномерное векторное подпространство. Образ $\exp \left(V_{2}\right)$ - это центр группы $\mathbb{H}^{n}$. Групповая операция определяется формулой

$$
(x, y, t) \cdot\left(x^{\prime}, y^{\prime}, t^{\prime}\right)=\left(x+x^{\prime}, y+y^{\prime}, t+t^{\prime}+2\left\langle y, x^{\prime}\right\rangle-2\left\langle x, y^{\prime}\right\rangle\right) .
$$

4.1.4. МЕТРИЧЕСКИЕ СТРУКТУРЫ НА ГРУППАХ КАРНО. Однородная норма $[70,71]$ на группе $\mathbb{G}$ - это непрерывная функция $\rho: \mathbb{G} \rightarrow[0, \infty)$, обладающая свойствами:

(a) $\rho(x)=0$ тогда и только тогда, когда $x=e$;

(b) $\rho(x)=\rho\left(x^{-1}\right)$ и $\rho\left(\delta_{t}(x)\right)=t \rho(x)$;

(c) существует постоянная $C>0$ такая, что $\rho(x \cdot y) \leq c(\rho(x)+\rho(y))$ для $\operatorname{Bcex} x, y \in \mathbb{G}$.

Естественно, что однородная норма определяется неоднозначно, однако любые две однородные нормы $\rho_{1}$ и $\rho_{2}$ эквивалентны [70] между собой: существуют числа $\alpha, \beta \in(0, \infty)$ такие, что $\alpha \leq \rho_{1}(x) / \rho_{2}(x) \leq \beta$ независимо от $x \in \mathbb{G} \backslash\{e\}$.

Однородная норма определяет однородную квазиметрику: для двух точек $x, y \in \mathbb{G}$, положим $\rho(x, y)=\rho\left(x^{-1} y\right)$. Квазиметрика обладает следующими свойствами, вытекающими из свойств (a)-(c) однородной нормы:

$\left(\mathrm{a}_{1}\right) \rho(x, y) \geq 0 ; \rho(x, y)=0$ тогда и только тогда, когда $x=y$;

$\left(\mathrm{b}_{1}\right) \rho(x, y)=\rho(y, x)$ и $\rho\left(\delta_{t} x, \delta_{t} y\right)=t \rho(x, y)$;

$\left(\mathrm{c}_{1}\right)$ для всех $x, y, z \in \mathbb{G}$ выполняется обобщенное неравенство треугольника $\rho(x, y) \leq C(\rho(x, z)+\rho(z, y))$ с постоянной $C>0$ из п. (с).

Из эквивалентности однородных норм $\rho_{1}$ и $\rho_{2}$ выводим эквивалентность метрик: $\alpha \rho_{2}(x, y) \leq \rho_{1}(x, y) \leq \beta \rho_{2}(x, y)$ для всех $x, y \in \mathbb{G}$.

Относительно метрики $\rho(x, y)$ задаются сферы $S_{\rho}(x, t)=\{y \in \mathbb{G}: \rho(x, y)=$ $t\}$ и шары $B_{\rho}(x, t)=\{y \in \mathbb{G}: \rho(x, y)<t\}$, причем сферы замкнуты, а шары открыты в топологии группы $\mathbb{G}$.

Далее фиксируем однородную норму элемента $x=\left(x_{1} ; \ldots ; x_{i} ; \ldots ; x_{m}\right) \in$ $\mathbb{R}^{N}, x_{i}=\left(x_{i 1}, \ldots, x_{i n_{i}}\right) \in V_{i}$, определенную по правилу

$$
\rho(x)=\max \left(\left|x_{1}\right|,\left|x_{2}\right|^{\frac{1}{2}}, \ldots,\left|x_{m}\right|^{\frac{1}{m}}\right) .
$$

где $\left|x_{i}\right|=\left(x_{i 1}^{2}+\cdots+x_{i n_{i}}^{2}\right)^{\frac{1}{2}}$ для $x_{i} \in V_{i}, i=1, \ldots, m$.

Предполагаем, что в алгебре Ли $\mathscr{G}$ задано скалярное произведение, относительно которого базисные левоинвариантные векторные поля $\left\{X_{i j}\right\}, 1 \leq i \leq m$, $1 \leq j \leq n_{i}$, ортонормированы.

Расстоянием Карно - Каратеодори $d(x, y)$ между двумя точками $x, y \in \mathbb{G}$ называется нижняя грань длин всех горизонтальных кривых с концевыми точками $x, y$, где длина касательного вектора измеряется выбранной римановой метрикой на группе $\mathbb{G}$, а горизонтальная кривая есть кусочно гладкий путь, касательный вектор к которому принадлежит $V_{1}$. Можно показать, что $d(x, y)$ всегда конечная левоинвариантная метрика, относительно которой группа автоморфизмов $\delta_{t}$ является группой растяжений с коэффициентом $t: d\left(\delta_{t} x, \delta_{t} y\right)=$ $t d(x, y)[69,70]$. По определению полагаем $d(x)=d(0, x)$. Можно показать, что $d(x)$ - однородная норма, поэтому расстояния $d(x, y)$ и $\rho(x, y)$ эквивалентны. Сферы и шары радиуса $t \geq 0$ в метрике Карно - Каратеодори будем обозначать символами $S_{c}(0, t)$ и $B_{c}(0, t)$ соответственно. 
Эквивалентность метрических функций $d(x, y)$ и $\rho(x, y)$ приводит к тому, что тождественное отображение между метрическими пространствами $(\mathbb{G}, d(\cdot, \cdot))$ и $(\mathbb{G}, \rho(\cdot, \cdot))$ квазиизометрично.

4.1.5. МЕРЫ НА ГРУППАХ КАРНО. Фиксируем далее биинвариантную меру Хаара на $\mathbb{G}$ (которая получается в результате переноса меры Лебега с алгебры Ли $\mathscr{G}$ на группу $\mathbb{G}$ посредством экспоненциального отображения, т. е. мера Хаара измеримого множества $A \subset \mathbb{G}$ равна мере Лебега множества $\exp ^{-1}(A)$ на алгебре $\mathscr{G}[70$, предложение 1.2$])$. Нормируем меру Хаара таким образом, чтобы мера Лебега шара $B_{c}(0,1)$ была равна 1 . Заметим, что нормирующий множитель в определении меры Хаусдорфа $\mathscr{H}^{N}(A)$ измеримого множества $A$ на пространстве $\mathbb{R}^{N}$ с евклидовой метрикой можно выбрать таким образом, чтобы мера Хаудорфа $\mathscr{H}^{N}(A)$ была равна его лебеговой мере. В силу изложенного, говоря в дальнейшем о мере Лебега множества $A \subset \mathbb{G}$, будем иметь в виду меру Хаудорфа $\mathscr{H}^{N}(A)$.

Отсюда имеем соотношение $\mathscr{H}^{N}\left(\delta_{t} A\right)=t^{\nu} \mathscr{H}^{N}(A)$ для измеримого множества $A \subset \mathbb{G}$, где число $\nu=\sum_{i=1}^{m} i n_{i}$ называется однородной размерностью группы $\mathbb{G}$.

Аналогично определенной в пространстве $\mathbb{R}^{n}$ мере Хаусдорфа (см. $\S 1$ ) рассмотрим меру Хаусдорфа на метрическом пространстве $(\mathbb{G}, d)$. Для $k \geq 0$, $\delta \in(0, \infty]$ и множества $A \subset \mathbb{G}$ зададим величину

$$
\mathscr{H}_{\delta}^{k}(A)=\frac{\omega_{k}}{2^{k}} \inf \left\{\sum_{i \in \mathbb{N}}\left(\operatorname{diam} A_{i}\right)^{k}: \operatorname{diam} A_{i}<\delta, A \subset \bigcup_{i \in \mathbb{N}} A_{i}\right\},
$$

где $\omega_{k}-$ нормирующий множитель, $\operatorname{diam} A_{i}=\sup \left\{d(x, y): x, y \in A_{i}\right\}$, а инфимум берется по всем счетным покрытиям $\left\{A_{i}\right\}$ множества $A$. Если $A$ не может быть покрыто счетным набором множеств указанного размера, то полагаем $\mathscr{H}_{\delta}^{k}(A)=\infty$. Предел $\mathscr{H}^{k}(A)=\lim _{\delta \rightarrow 0} \mathscr{H}_{\delta}^{k}(A)$ существует и называется $k$-мерной мерой Хаусдорфа множества $A$ на $(\mathbb{G}, d)$.

Выберем нормирующий множитель $\omega_{\nu}$ в определении меры Хаусдорфа так, что $\mathscr{H}^{\nu}\left(B_{c}(0,1)\right)=1$, где $B_{c}(0,1)$ - шар в метрике Карно - Каратеодори. Тогда $\mathscr{H}^{\nu}\left(B_{c}(0, r)\right)=r^{\nu}$. Более того, если $E \subset \mathbb{G}-$ измеримое множество, то $\mathscr{H}^{\nu}\left(\delta_{t} E\right)=t^{\nu} \mathscr{H}^{\nu}(E)$. Отметим, что однородная размерность $\nu$ группы $\mathbb{G}$ равно размерности Хаусдорфа пространства $(\mathbb{G}, d)$.

Из-за подходяще выбранных нормирующих множителей $\nu$-мерная мера Хаусдорфа $\mathscr{H}^{\nu}(A)$ измеримого множества $A \subset \mathbb{G}$ равна его мере Лебега $\mathscr{H}^{N}(A)$.

4.1.6. ГОРИЗОНТАЛЬНЫЕ СЛОЕНИЯ НА ГРУППАХ КАРНО. Фиксируем $1 \leq$ $j \leq n_{1}$ и рассмотрим семейство кривых $\Gamma_{j}$, образующих гладкое слоение открытого множества $A \subset \mathbb{G}$. Роль слоя $\gamma \in \Gamma_{j}$ играют интегральные кривые горизонтального векторного поля $X_{1 j} \in V_{1}$. Если соответствующий этому полю поток обозначить символом $g_{s}$, то слой имеет вид $\gamma(s)=g_{s}(p)=p \exp s X_{1 j}$, где $p$ принадлежит поверхности $P$, трансверсальной к векторному полю $X_{1 j}$, а параметр $s-$ интервалу $I \subset \mathbb{R}$. Предполагается, что слоение $\Gamma_{j}$ множества $A$ снабжено мерой $d \gamma_{j}$, удовлетворяющей соотношениям

$$
c_{7} r^{\nu-1} \leq \int_{\gamma \in \Gamma_{j}, \gamma_{j} \cap B_{c}(x, r) \neq \varnothing} d \gamma \leq c_{8} r^{\nu-1}
$$

для достаточно малых шаров $B_{c}(x, r) \subset \mathbb{G}$ с постоянными $c_{7}$ и $c_{8}$, не зависящими от $B_{c}(x, r)$. Для слоения, определяемого векторным полем $X_{1 j} \in V_{1}$, мера $d \gamma_{j}$ 
может быть получена как внутреннее произведение $i\left(X_{1 j}\right)$ векторного поля $X_{1 j}$ с биинвариантной формой объема $d x(\text { см. [67] })^{9)}$.

Горизонтальные слоения на группах Карно - удобное понятие при определении абсолютно непрерывных функций (или отображений) на группах Карно: аналогом известного в евклидовом пространстве выражения «отображение абсолютно непрерывно на почти всех линиях параллельных координатным осям» на группах Карно будет выражение «отображение абсолютно непрерывно на $d \gamma_{j}$-почти всех линиях горизонтального слоения $\Gamma_{j}, 1 \leq j \leq n_{1}$ ».

4.1.7. ДИФФЕРЕНЦИРУЕМОСТЬ НА ГРУППАХ КАРНО. Пусть заданЫ две группы Карно $\mathbb{G}, \widetilde{\mathbb{G}}$ и $D$ - область в $\mathbb{G}$. Отображение $\varphi: D \rightarrow \widetilde{\mathbb{G}}$ называется $\mathscr{P}$ дифференцируемым [69] в точке $x \in D$, если существует гомоморфизм $L: \mathbb{G} \rightarrow \widetilde{\mathbb{G}}$ групп Карно такой, что $L\left(\exp V_{1}\right) \subset \exp \widetilde{V}_{1}$ и «разностное отношение»

$$
\tilde{\delta}_{t}^{-1}\left(\varphi(x)^{-1} \varphi\left(x \delta_{t} u\right)\right) \quad \text { сходится к } L(u)
$$

при $t \rightarrow 0+$ равномерно по $u \in B_{c}(0,1)$. Здесь $\tilde{\delta}_{t}-$ однопараметрическая группа растяжений на $\widetilde{\mathbb{G}}$, а $\widetilde{V}_{1}$ - горизонтальное подпространство алгебры Ли $\widetilde{\mathscr{G}}$ группы $\widetilde{\mathbb{G}}$.

Пансю доказал [69], что всякое определенное на открытом множестве $D$ липшицево отображение $\varphi: D \rightarrow \widetilde{\mathbb{G}} \mathscr{P}$-дифференцируемо $\mathscr{H}^{\nu}$-п. в. в $D$.

Специализируя сходимость в (47) в других топологиях, приходим к различным понятиям дифференцируемости. Так, сходимость в (47) по мере на шаре $B_{c}(0,1)$ приводит к понятию аппроксимативной дифференцируемости (см. [73]), а сходимость в топологии пространства Соболева - к дифференцируемости в топологии пространства Соболева (см. [56]).

4.2. Классы Соболева на группах Карно. Пусть $D$ - область в $\mathbb{G}$. Локально суммируемая функция $f: D \rightarrow \mathbb{R}$ принадлежит классу Соболева $L_{p}^{1}(D)$, $p \in[1, \infty]$, если обобщенные производные $X_{1 j} f, j=1, \ldots, n$, вдоль векторных полей $X_{1 j}$ принадлежат $L_{p}(D)$. Пространство $L_{p}^{1}(D), p \in[1, \infty]$, рассматривается с полунормой

$$
\left\|f \mid L_{p}^{1}(D)\right\|=\left(\int_{D}|\nabla \mathscr{L} f|^{p}(x) d \mathscr{H}^{\nu}(x)\right)^{\frac{1}{p}},
$$

где вектор $\nabla_{\mathscr{L}} f(x)=\left(X_{11} f(x), \ldots, X_{1 n_{1}} f(x)\right) \in V_{1}$ называется субградиентом функции $f$.

Пусть заданы две группы Карно $\mathbb{G}, \mathbb{G}^{\prime}$ и $D$ - область в $\mathbb{G}$. Отображение $\varphi: D \rightarrow \mathbb{G}^{\prime}$ называется абсолютно непрерывным на линиях $(\varphi \in \mathrm{ACL}(D))$, если для любой области $U, \bar{U} \subset D$, и слоения $\Gamma_{j}$, определяемого левоинвариантным векторным полем $X_{1 j}, j=1, \ldots, n_{1}$, отображение $\varphi$ абсолютно непрерывно на $\gamma \cap U$ относительно $\mathscr{H}^{1}$-меры Хаусдорфа для $d \gamma_{j}$-п. в. кривых $\gamma \in \Gamma_{j}$. Для такого отображения $\mathscr{H}^{\nu}$-п. в. в $D$ существуют $[69$, предложение 4.1] производные $X_{1 j} \varphi$ вдоль векторных полей $X_{1 j}, j=1, \ldots, n_{1}$, такие, что $X_{1 j} \varphi(x) \in V_{1}(x)$.

Матрица $D_{h} \varphi(x)=\left(X_{1 i} \varphi_{1 j}(x)\right), i=1, \ldots, n_{1}, j=1, \ldots, n_{1}^{\prime}$, определенная п. в. в $D$, определяет линейный оператор $D_{h} \varphi(x): V_{1} \rightarrow V_{1}^{\prime}, \operatorname{dim} V_{1}^{\prime}=n_{1}^{\prime}$, горизонтального пространства $V_{1}$ в горизонтальное пространство $V_{1}^{\prime}$ группы $\mathbb{G}^{\prime}$, назы-

\footnotetext{
${ }^{9)}$ Если $d x$ - форма объема на $\mathbb{G}($ степени $N)$, то $i\left(X_{1 j}\right)$ - форма степени $N-1$, которая на гладких векторных полях $Y_{1}, Y_{2}, \ldots, Y_{N-1}$, определенных на $\mathbb{G}$, принимает значение $i\left(X_{1 j}\right)\left(Y_{1}, Y_{2}, \ldots, Y_{N-1}\right)=d x\left(X_{1 j}, Y_{1}, Y_{2}, \ldots, Y_{N-1}\right)$.
} 
ваемый горизонталъным дифференииалом отображения $\varphi$ в точке $x ;\left|D_{h} \varphi(x)\right|$ обозначает норму этого оператора.

Алгебраически-аналитическая специфика группы Карно проявляется в том, что горизонтальный дифференциал $D_{h} \varphi(x): V_{1} \rightarrow V_{1}^{\prime}$ порождает [73, теорема 1.2] гомоморфизм $D \varphi(x): \mathbb{G} \rightarrow \mathbb{G}^{\prime}$ групп Карно, называемый $\mathscr{P}$-дифференциалом (см. разд. 4.1.7), при этом их нормы оцениваются одна через другую: $\left|D_{h} \varphi(x)\right| \leq|D \varphi(x)| \leq C\left|D_{h} \varphi(x)\right|$, где $C$ зависит только от алгебраической структуры группы $\mathbb{G}$. Известно [73, теорема 1.2$]$, что для п. в. $x \in D$ гомоморфизм $D \varphi(x)$ является аппроксимативным дифференциалом отображения $\varphi: D \rightarrow \mathbb{G}^{\prime}$, если $\varphi \in W_{1, \mathrm{loc}}^{1}(D)$.

Отображение $\varphi: D \rightarrow \mathbb{G}^{\prime}$ принадлежит классу Соболева $W_{p, \text { loc }}^{1}(D), p \in$ $[1, \infty)$, если $\varphi \in \operatorname{ACL}(D)$ и на каждой области $U \subset D, \bar{U} \subset D$, величина

$$
\left\|\varphi\left|W_{p}^{1}(U)\|=\| \rho(\varphi(\cdot))\right| L_{p}(U)\right\|+\left(\int_{U}\left|D_{h} \varphi(x)\right|^{p} d \mathscr{H}^{\nu}(x)\right)^{\frac{1}{p}}
$$

конечная. Описания отображений классов Соболева, эквивалентные вышеприведенному, см. в [73].

4.3. Замена переменной для отображений ACL-классов на группах Карно. Далее рассматриваем совпадающие группы $\mathbb{G}$ и $\mathbb{G}^{\prime}$ и отображение $\varphi$ : $D \rightarrow \mathbb{G}, D \subset \mathbb{G}$, принадлежащее классу Соболева $W_{p, \text { loc }}^{1}(D), p \in[1, \infty)$.

Определитель $\operatorname{det} D \varphi(x)$ матрицы гомоморфизма $D \varphi(x)$ называется так же, как и в $\mathbb{R}^{n}$, якобианом отображения $\varphi$ в точке $x$. Геометрический смысл якобиана аналогичен (11): если $\varphi: D \rightarrow D^{\prime}, D, D^{\prime} \subset \mathbb{G},-$ гомеоморфизм класса Соболева, то

$$
D \ni x \mapsto \lim _{r \rightarrow 0} \frac{\mathscr{H}^{\nu}\left(\varphi\left(B_{c}(x, r)\right)\right)}{\mathscr{H}^{\nu}\left(B_{c}(x, r)\right)}=\lim _{r \rightarrow 0} \frac{\mathscr{H}^{N}\left(\varphi\left(B_{c}(x, r)\right)\right)}{\mathscr{H}^{N}\left(B_{c}(x, r)\right)}=|\operatorname{det} D \varphi(x)|
$$

для $\mathscr{H}^{\nu}$-п. в. $x \in D$. Равенства (48) можно получить с помощью приводимой ниже формулы замены переменной (50) и теоремы Лебега о дифференцировании интеграла на группе Карно (см., например, [47, следствие 3]).

ОПреДЕЛЕНИЕ 37. Для отображения $\varphi: D \rightarrow \mathbb{G}$ на группе Карно класса $W_{p, \text { loc }}^{1}(D)$ определим борелевское множество $Z=\{x \in D: \operatorname{det} D \varphi(x)=0\}$ нулей якобиана и множество сингулярности

$$
\Sigma=D \backslash\{x \in D: \text { определен аппроксимативный дифференциал } D \varphi(x)\}
$$

меры нуль, которое можно считать борелевским. Очевидно $Z \cap \Sigma=\varnothing$.

Аналогично определению 17 зададим множества $Z^{\prime}=\varphi(\Sigma)$ и $\Sigma^{\prime}=\varphi(Z)$.

Формулы замены переменной в интеграле Лебега на группе Карно можно доказать, повторяя дословно рассуждения доказательства предложения 15 из [52] с той лишь разницей, что применяемые в этом доказательстве утверждения из [49] следует заменить соответствующими теоремами из [73].

Приведем здесь лишь одну простую формулу из [73, следствие 5.1], используемую ниже.

Предложение 38. Если гомеоморфизм $\varphi: D \rightarrow D^{\prime}, D, D^{\prime} \subset \mathbb{G}$, принадлежит классу Соболева $W_{1, \mathrm{loc}}^{1}(D)$ (или классу $\left.\mathrm{ACL}(D)\right)$, то вне борелевского множества $\Sigma \subset D($ см. (49)) нулевой меры отображение $\varphi: D \backslash \Sigma \rightarrow \mathbb{G}$ обладает $\mathscr{N}$-свойством Лузина и для любой интегрируемой функции $u: D^{\prime} \rightarrow \mathbb{R}$ верна 
формула

$$
\int_{D} u(\varphi(x))|\operatorname{det} D \varphi(x)| d \mathscr{H}^{\nu}(x)=\int_{D^{\prime} \backslash Z^{\prime}} u(y) d \mathscr{H}^{\nu}(y), \quad \text { где } Z^{\prime}=\varphi(\Sigma) .
$$

\section{4. Свойства регулярности гомеоморфизмов классов Соболева} на группах Карно. Будем говорить, что отображение $\varphi: D \rightarrow \mathbb{G}$ на группе Карно, принадлежащее классу Соболева $W_{1, \mathrm{loc}}^{1}(D)$ или $\mathrm{ACL}(D)$, имеет конечное искажение, если $D \varphi(x)=0$ п. в. на множестве $Z=\{x \in D: \operatorname{det} D \varphi(x)=0\}$ нулей якобиана.

4.4.1. ГОМЕОМОРФИЗМ КЛАССА СОБОЛЕВА НА ГРУППЕ КАРНО КАК ОПЕРАТОР КОМПОЗИции. В следующем утверждении обобщаем на группы Карно утверждение 1.

Утверждение 39. Пусть $D, D^{\prime}$ - области на группе Карно $\mathbb{G}$, а $\varphi: D \rightarrow$ $D^{\prime}$ - гомеоморфизм класса Соболева $W_{p, \mathrm{loc}}^{1}(D), 1 \leq p<\infty$, имеющий конечное искажение. Тогда весовая функция, определяемая соотношением

$$
D^{\prime} \ni y \mapsto \omega(y)= \begin{cases}\frac{\mid D \varphi\left(\left.\varphi^{-1}(y)\right|^{p}\right.}{\left|\operatorname{det} D \varphi\left(\varphi^{-1}(y)\right)\right|}, & \text { если } y \in D^{\prime} \backslash\left(Z^{\prime} \cup \Sigma^{\prime}\right), \\ 1 & \text { иначе }\end{cases}
$$

локально суммируема: $\omega \in L_{1, \mathrm{loc}}\left(D^{\prime}\right)$, и $\Sigma^{\prime}=\varphi(z)$, оператор композиции

$$
\varphi^{*}: L_{p}^{1}\left(D^{\prime} ; \omega\right) \cap \operatorname{Lip}_{l}\left(D^{\prime}\right) \rightarrow L_{p}^{1}(D), \quad 1 \leq p<\infty,
$$

ограничен, причем $\left\|\varphi^{*}\right\| \leq\left\|K_{p, p}^{1, \omega}(\cdot) \mid L_{\infty}(D)\right\|=1$, где операторная функция искажения $K_{p, p}^{1, \omega}(\cdot)$ определена ниже в (53).

ДокАзАтЕльство. Проверим, что весовая функция (51) локально суммируема, операторная функция искажения $K_{p, p}^{1, \omega}(\cdot)$ (см. ниже (53)) принадлежит $L_{\infty}(D)$ и оператор композиции $(52)$ ограничен.

Применяя формулы замены переменной (50), выводим

$$
\begin{gathered}
\int_{\varphi(W) \backslash\left(Z^{\prime} \cup \Sigma^{\prime}\right)} \omega(y) d \mathscr{H}^{\nu}(y)=\int_{\varphi(W) \backslash\left(Z^{\prime} \cup \Sigma^{\prime}\right)} \frac{\left|D \varphi\left(\varphi^{-1}(y)\right)\right|^{p}}{\left|\operatorname{det} D \varphi\left(\varphi^{-1}(y)\right)\right|} d \mathscr{H}^{\nu}(y) \\
=\int_{W \backslash(Z \cup \Sigma)}|D \varphi(x)|^{p} d \mathscr{H}^{\nu}(x)<\infty
\end{gathered}
$$

для компактно вложенной области $W \Subset D$. Таким образом, $\omega \in L_{1, \mathrm{loc}}\left(D^{\prime}\right)$.

Операторная функция искажения

$$
D \ni x \mapsto K_{p, p}^{1, \omega}(x, \varphi)= \begin{cases}\frac{|D \varphi(x)|}{|\operatorname{det} D \varphi(x)|^{\frac{1}{p}} \omega^{\frac{1}{p}}(\varphi(x))}, & \text { если } \operatorname{det} D \varphi(x) \neq 0, \\ 0, & \text { если } \operatorname{det} D \varphi(x)=0,\end{cases}
$$

принадлежит $L_{\infty}(D)$. Более того, $\left\|K_{p, p}^{1, \omega}(\cdot) \mid L_{\infty}(D)\right\|=1$.

Для оценки нормы оператора композиции (52) применяем формулу замены 
переменной (50) (см. ниже интеграл (54)):

$$
\begin{aligned}
\left\|u \circ \varphi \mid L_{p}^{1}(D)\right\|^{p} & \leq \int_{D}|\nabla \mathscr{L}(u \circ \varphi)(x)|^{p} d \mathscr{H}^{\nu}(x) \\
& \leq \int_{D}|\nabla \mathscr{L} u(\varphi(x))|^{p} \frac{|D \varphi(x)|^{p}}{|\operatorname{det} D \varphi(x)|}|\operatorname{det} D \varphi(x)| d \mathscr{H}^{\nu}(x) \\
& \leq \int_{D^{\prime}}|\nabla \mathscr{L} u(y)|^{p} \frac{\left|D \varphi\left(\varphi^{-1}(y)\right)\right|^{p}}{\left|\operatorname{det} D \varphi\left(\varphi^{-1}(y)\right)\right| \omega(y)} \omega(y) d \mathscr{H}^{\nu}(y) \\
& \leq\left\|K_{p, p}^{1, \omega}(\cdot)\left|L_{\infty}(D)\left\|^{p} \cdot\right\| u\right| L_{p}^{1}\left(D^{\prime} ; \omega\right)\right\|^{p},
\end{aligned}
$$

справедливую для любой функции $u \in L_{p}^{1}\left(D^{\prime} ; \omega\right) \cap \operatorname{Lip}_{l}\left(D^{\prime}\right)$. Отсюда $\left\|\varphi^{*}\right\| \leq$ $\left\|K_{p, p}^{1, \omega}(\cdot) \mid L_{\infty}(D)\right\|=1$.

4.4.2. ГОМЕОМОРФИЗМЫ КЛАССОВ СОБОЛЕВА НА ГРУППЕ КАРНО И РЕГУлярность овРАтных к ним. В следующем утверждении обобщаем на группы Карно утверждение 2.

Утверждение 40. Пусть $D, D^{\prime}$ - области на группе Карно $\mathbb{G}$, а $\varphi: D \rightarrow$ $D^{\prime}$ - гомеоморфизм класса Соболева $W_{p, \mathrm{loc}}^{1}(D), \nu-1<p<\infty$, имеющий конечное искажение.

Тогда обратный гомеоморфизм $f=\varphi^{-1}: D^{\prime} \rightarrow D$ обладает следующими свойствами:

1) $f$ дифференцируем $\mathscr{H}^{\nu}$-п. в. в области $D^{\prime}$;

2) $f$ имеет конечное искажение;

3) для $\mathscr{H}^{\nu}$-п. в. $y \in D^{\prime}$ справедлива оценка

$$
|D f(y)| \leq c_{9}|\operatorname{det} D f(y)|^{\frac{p-(\nu-1)}{p}} \omega(y)^{\frac{\nu-1}{p}} ;
$$

4) для любого открытого множества $U \subset D^{\prime}$ имеет место неравенство

$$
\int_{U}|D f(y)| d \mathscr{H}^{\nu}(y) \leq c_{9} \mathscr{H}^{\nu}(f(U))^{\frac{p-(n-1)}{p}} \omega(U)^{\frac{\nu-1}{p}}
$$

с весовой функцией $(51)$ и постоянной $c_{9}=2^{\nu}\left(\frac{1}{c_{10}}\right)^{\frac{\nu-1}{p}}$ (последнее равенство обеспечено нормировкой $\left.\mathscr{H}^{\nu}(B(0, r))=r^{\nu}\right)$, где величина $c_{10}$ определена ниже в неравенстве (59).

5) принадлежит классу Соболева $W_{1, \text { loc }}^{1}\left(D^{\prime}\right)$.

Утверждение 40 можно доказать, следуя схеме доказательств теорем 21 и 23. Ниже приведем основные рассуждения доказательства утверждения 40, акцентируя внимание на специфику геометрии групп Карно.

4.4.3. ОцЕНКИ ЕМКОСТИ НА ГРУППАХ КАРно. В основе доказательства утверждения 40 лежат оценки для емкости двух конденсаторов на группе Карно, аналогичные рассмотренным в разд. 2.1.

Непрерывная функция $u: D \rightarrow \mathbb{R}$ класса $W_{1, \text { loc }}^{1}(D)$ называется допустимой для конденсатора $E=(F, U)$, если $u \equiv 1$ на $F$ и $u \equiv 0$ вне $U$. Применяем символ $\mathscr{A}(E)$ для совокупности допустимых для конденсатора $E=(F, U)$ функций.

Далее обобщаем на группы Карно понятие емкости, определенное в (6) в евклидовых пространствах. 
Емкость конденсатора $E=(F, U)$ в пространстве $L_{q}^{1}(D), q \in[1, \infty)$, на группе Карно определим как величину

$$
\operatorname{cap}\left(E ; L_{q}^{1}(D)\right)=\inf _{u \in \mathscr{A}(E) \cap L_{q}^{1}(D)}\left\|u \mid L_{q}^{1}(D)\right\|^{q},
$$

где инфимум берется по всем функциям пересечения $\mathscr{A}(E) \cap L_{q}^{1}(D)$.

Весовые функции, весовые пространства Соболева $L_{p}^{1}\left(D^{\prime} ; \omega\right)$ и пространство локально липшицевых функций $\operatorname{Lip}_{l}\left(D^{\prime}\right)$ на области $D^{\prime}$ метрического пространства $(\mathbb{G}, d)$ определяются по аналогии с евклидовым пространством $\mathbb{R}^{n}$ (см. (5)).

Весовая емкость конденсатора $E=(F, U) \subset D^{\prime}$ в пространстве $L_{p}^{1}\left(D^{\prime} ; \omega\right)$ на группе Карно - это величина

$$
\operatorname{cap}\left(E ; L_{p}^{1}\left(D^{\prime} ; \omega\right)\right)=\inf _{u \in \mathscr{A}(E) \cap \operatorname{Lip}_{l}\left(D^{\prime}\right)}\left\|u \mid L_{p}^{1}\left(D^{\prime} ; \omega\right)\right\|^{p},
$$

где инфимум берется по всем функциям $u \in \mathscr{A}(E) \cap \operatorname{Lip}_{l}\left(D^{\prime}\right)$.

Расстояние между двумя непустыми множествами $A_{1}, A_{2} \subset \mathbb{G}$ на группе Карно равно $\operatorname{dist}\left(A_{1}, A_{2}\right)=\inf \left\{d_{c}(x, y): x \in A_{1}, y \in A_{2}\right\}$.

Ниже применяется обозначение $\omega(E)=\int_{E} \omega(y) d \mathscr{H}^{\nu}(y)$ для измеримого множества $E \subset \mathbb{G}$.

Лемма 41. При $1 \leq p<\infty$ для весовой емкости конденсатора $E=(F, U)$ в $D^{\prime}$ справедлива оценка сверху

$$
\operatorname{cap}\left(E ; L_{p}^{1}(U ; \omega)\right) \leq \frac{\omega(U \backslash F)}{\operatorname{dist}(F, \partial U)^{p}} .
$$

ДоКАЗАТЕЛЬСТво этого свойства аналогично доказательству леммы 19 с той лишь разницей, что вместо евклидова расстояния надо использовать расстояние Карно - Каратеодори.

Лемма $42[18$, лемма 5]. Пусть $\nu-1<q<\infty$. Для конденсатора $E=$ $(F, U)$ в $D$ при условии связности множества $F$ имеет место неравенство

$$
\operatorname{cap}^{\nu-1}\left(E ; L_{q}^{1}(U)\right) \geq c_{10}^{\nu-1} \frac{(\operatorname{diam} F)^{q}}{\mathscr{H}^{\nu}(U)^{q-(\nu-1)}},
$$

где $c_{10}$ - постоянная, зависящая только от $q$ и геометрии группы.

4.4.4. ДоКАЗАТЕЛЬСТво УтВЕРЖДЕНИЯ 40. I. На этом шаге установим дифференцируемость отображения $f$. Воспользуемся схемой доказательства теоремы 21. С каждой точкой $y \in D^{\prime}$ свяжем сферический конденсатор $E_{r}=$ $\left(\overline{B_{c}(y, r)}, B_{c}(y, 2 r)\right)$, где $B_{c}(y, 2 r) \subset D^{\prime}$, т. е. конденсатор, у которого граница состоит из двух компонент связности: двух концентрических сфер. Учитывая норму оператора $\left\|\varphi^{*}\right\| \leq 1$, имеем

$$
\operatorname{cap}\left(f\left(E_{r}\right) ; L_{p}^{1}\left(f\left(B_{c}(y, 2 r)\right)\right)\right) \leq \operatorname{cap}\left(E_{r} ; L_{p}^{1}(U ; \omega)\right) \leq \frac{\omega\left(B_{c}(y, 2 r)\right)}{r^{p}} .
$$

Используя для оценки емкости слева неравенство (59) при $q=p$ :

выводим

$$
c_{10} \frac{\left(\operatorname{diam} f\left(\overline{B_{c}(y, r)}\right)\right)^{\frac{p}{\nu-1}}}{\mathscr{H}^{\nu}\left(f\left(B_{c}(y, 2 r)\right)\right)^{\frac{p-(\nu-1)}{\nu-1}}} \leq \operatorname{cap}\left(f\left(E_{r}\right) ; L_{p}^{1}\left(f\left(B_{c}(y, 2 r)\right)\right)\right),
$$

$$
\begin{aligned}
\frac{\operatorname{diam} f(\overline{B(y, r)})}{r} \leq & \frac{2^{\nu} c_{10}^{\frac{1-\nu}{p}}}{(2 r)^{\nu}} \mathscr{H}^{\nu}(f(B(y, 2 r)))^{\frac{p-(\nu-1)}{p}} \omega(B(y, 2 r))^{\frac{\nu-1}{p}} \\
& =2^{\nu} c_{10}^{\frac{1-\nu}{p}}\left(\frac{\mathscr{H}^{\nu}(f(B(y, 2 r)))}{\mathscr{H}^{\nu}(B(y, 2 r))}\right)^{\frac{p-(\nu-1)}{p}}\left(\frac{\omega(B(y, 2 r)}{\mathscr{H}^{\nu}(B(y, 2 r))}\right)^{\frac{\nu-1}{p}} .
\end{aligned}
$$


Устремляя $r$ к 0 , для $\mathscr{H}^{\nu}$-п. в. $y \in D^{\prime}$ получаем

$$
\varlimsup_{z \rightarrow y} \frac{d(f(z), f(y))}{d(z, y)} \leq 2^{\nu}\left(\frac{1}{c_{10}}\right)^{\frac{\nu-1}{p}} \mathscr{V}_{\nu}^{\prime}(y)^{\frac{p-(\nu-1)}{p}} \omega(y)^{\frac{\nu-1}{p}}
$$

где значение $\mathscr{V}_{\nu}^{\prime}(y)=\lim _{r \rightarrow 0} \frac{\mathscr{H}^{\nu}(f(B(y, r)))}{\mathscr{H}^{\nu}(B(y, r))}$, равное объемной производной, конечно $\mathscr{H}^{\nu}$-п. в. в $D^{\prime}$ (см. (48)). По теореме Лебега о дифференцируемости интеграла $\lim _{r \rightarrow 0} \frac{\omega(B(y, 2 r)}{\mathscr{H}^{\nu}(B(y, 2 r))}=\omega(y)$ для $\mathscr{H}^{\nu}$-п. в. $y \in D^{\prime}$ (см., например, [72, гл. $1, \S 3.1$, следствие; 46; 47, следствие 3]).

Поскольку правая часть (60) конечна $\mathscr{H}^{\nu}$-п. в. в $D^{\prime}$, по теореме типа Степанова на группах Карно (см. [73, теорема 3.1]) отображение $f$ дифференцируемо $\mathscr{H}^{\nu}$-п. в. в $D^{\prime}$. Известно, что в точках дифференцируемости отображения $f$ левая часть в (60) равна $|D f(y)|$ (см., например, [73, следствие 2.1]), а $\mathscr{V}_{\nu}^{\prime}(y)=|\operatorname{det} D f(y)|(48)$.

II, III. С учетом вышеизложенного перепишем неравенство (60) следующим образом:

$$
|D f(y)| \leq c_{9}|\operatorname{det} D f(y)|^{\frac{p-(\nu-1)}{p}} \omega(y)^{\frac{\nu-1}{p}},
$$

где $c_{9}=2^{\nu}\left(\frac{1}{c_{10}}\right)^{\frac{\nu-1}{p}}$. Отсюда получаем поточечную оценку (56). Далее, очевидно, верно следующее: $D f(y)=0$ на множестве $Z^{\prime}=\left\{y \in D^{\prime}: \operatorname{det} D f(y)=0\right\}$ нулей якобиана $\operatorname{det} D f(y)$ всюду, за исключением множества нулевой $\mathscr{H}^{\nu}$-меры. Следовательно, $f$ имеет конечное искажение.

IV. Для доказательства неравенства (57) следует проинтегрировать (56) и применить неравенство Гёльдера с учетом $\frac{p-(\nu-1)}{p}+\frac{\nu-1}{p}=1$ :

$$
\int_{U}|D f(y)| d \mathscr{H}^{\nu}(y) \leq c_{9}\left(\int_{U}|\operatorname{det} D f(y)| d \mathscr{H}^{\nu}(y)\right)^{\frac{p-(\nu-1)}{p}}\left(\int_{U} \omega(y) d \mathscr{H}^{\nu}(y)\right)^{\frac{\nu-1}{p}} .
$$

Поскольку $\int_{U}|\operatorname{det} D f(y)| d \mathscr{H}^{\nu}(y) \leq \mathscr{H}^{\nu}(f(U))$ (см. (50)), выводим (57). Таким образом, $|D f(y)|$ локально суммируема в $D^{\prime}$.

V. Остается доказать свойство 5. Для этого достаточно проверить, что $f \in$ $\mathrm{ACL}\left(D^{\prime}\right)$. С учетом локальной суммируемости горизонтальных производных (см. (57)) получим требуемое (см. эквивалентное описание отображений $f \in$ $W_{1, \text { loc }}^{1}\left(D^{\prime}\right)$ на группах Карно в [73, предложение 4.2]).

Отметим, что приводимое ниже доказательство абсолютной непрерывности отображений на группах Карно новое и существенно отличается от такового в евклидовом пространстве (см. доказательство теоремы 23).

ШАГ 1. Напомним, что символ $B_{\rho}(y, t)$ обозначает шар в метрическом пространстве $(\mathbb{G}, \rho)($ см. (45)) с центром в точке $y$ радиуса $t$.

Для доказательства $f \in \mathrm{ACL}\left(D^{\prime}\right)$ фиксируем $1 \leq j \leq n$. Для некоторого числа $^{10)} M \in(0, \infty)$ определим множества

$$
\begin{gathered}
P_{j 0}(0, t)=\left\{y \in B_{\rho}(0, t): y_{j}=0\right\}, \quad t \in(0, M), \\
P_{j}(0, t)=\left\{z=y \exp y_{j} X_{1 j}: y \in P_{j 0}(0, t),\left|y_{j}\right|<t,\right\}, \quad t \in(0, M), \\
P_{j}(w, t)=w P_{j}(0, t) \subset D^{\prime} \quad \text { для } w \in D^{\prime} \text { и } t \in(0, M) .
\end{gathered}
$$

10) Значение числа $M$ обусловлено необходимостью обеспечить справедливость оценок (65)-(67); точное значение числа $M$ для доказательства не требуется. 
Заметим, что множество $P_{j}(0, t)$ является шаром в метрике $\rho_{j}(x, y)$, определяемой ниже: $y \in P_{j}(0, t)$ тогда и только тогда, когда $\rho_{j}(y)<t$, где однородная норма $\rho_{j}$ определена условием:

$$
\mathscr{G} \ni y \mapsto \rho_{j}(y)=\max \left(\left\{\rho(y): y_{j}=0\right\},\left|y_{j}\right|\right)
$$

на алгебре Ли $\mathscr{G}$, а на группу переносится посредством глобальной системы координат $\exp (y)=\exp \left(\sum y_{i k} X_{i j}\right) \cdot \exp \left(y_{j} X_{1 j}\right)$, где индексы суммирования изменяются в пределах $1 \leq i \leq m, 1 \leq k \leq n_{1}, k \neq j$ при $i=1,1 \leq k \leq n_{i}$ при $i \geq 2$. Следовательно, квазиметрика $\rho_{j}(x, y)$ эквивалентна в смысле п. 4.1.4 как квазиметрике $\rho(x, y)$, так и метрике $d_{c}(x, y)$.

Применяя лемму о покрытии из $[70$, лемма 1.66$]$ в случае $D^{\prime}=\mathbb{G}$ или из $[72$, $\S 3.1$, лемма 2] в случае $D^{\prime} \neq \mathbb{G}$, выводим существование не более чем счетного семейства

$$
\left\{P_{j}\left(w_{k}, t_{k}\right): P_{j}\left(0, t_{k}\right) \Subset P_{j}(0, M)\right\}, \quad k \in \mathbb{N},
$$

множеств, указанного в (61) вида, покрывающих $D^{\prime}: D^{\prime}=\bigcup_{k \in \mathbb{N}} P_{j}\left(w_{k}, t_{k}\right)$.

Поэтому достаточно доказать абсолютную непрерывность отображения $f$ на пересечении $\mathscr{H}^{\nu-1}$-п. в. интегральных линий горизонтального векторного поля $X_{1 j}$ с шаром $P_{j}\left(w_{k}, t_{k}\right)$ (здесь $\mathscr{H}^{\nu-1}-$ мера Хаусдорфа на поверхности $w_{k} P_{j 0}\left(0, t_{k}\right)$, трансверсальной к слоению $\Gamma_{j}$, см. (46)). Так как $k \in \mathbb{N}$ и $j=1, \ldots, n$ произвольные, ACL-свойство отображения $f$ на области $D^{\prime}$ будет доказано.

ШАГ 2. Фиксируем произвольное множество $P_{j}\left(w_{k}, t_{k}\right)$ из семейства (63). Изучение вопроса об абсолютной непрерывности отображения $f: P_{j}\left(w_{k}, t_{k}\right) \rightarrow$ $D^{\prime}$ сводим к изучению вопроса об абсолютной непрерывности композиции $f \circ$ $l_{w_{k}}: P_{j}\left(0, t_{k}\right) \rightarrow D^{\prime}$, где $l_{w_{k}}-$ левый сдвиг: $\mathbb{G} \ni x \mapsto w_{k} \cdot x$.

Во избежание громоздких формул введем следующие обозначения:

$$
Q_{0}=P_{j 0}\left(0, t_{k}\right), \quad Q=P_{j}\left(0, t_{k}\right) .
$$

Очевидно, часть плоскости $Q_{0} \subset\left\{y \in \mathbb{G}: y_{1 j}=0\right\}$ трансверсальна слоению $\Gamma_{j}$.

Рассмотрим ограничение $\tilde{\rho}=\left.\rho\right|_{Q_{0}}$ метрики $\rho$ на $Q_{0}$. Отметим применяемые ниже свойства метрической структуры $\left(Q_{0}, \tilde{\rho}\right)$ и мер $\mathscr{H}^{\nu-1}, \mathscr{H}^{N-1}$ на $Q_{0}$.

1. Метрическая функция $\tilde{\rho}$ является квазиметрикой в смысле п. 4.1.4, т. е. функция $\tilde{\rho}: Q_{0} \times Q_{0} \rightarrow[0, \infty)$ обладает свойствами $\left(a_{1}\right)-\left(c_{1}\right)$ п. 4.1.4 для всех точек $x, y \in Q_{0}$.

2. Мера Хаусдорфа $\mathscr{H}^{\nu-1}$ (см. п. 4.1.5) удовлетворяет условию удвоения: для шара $B_{\tilde{\rho}}(y, t)$ и меры Хаусдорфа $\mathscr{H}^{\nu-1}$ на $Q_{0}$ справедливо соотношение

$$
\mathscr{H}^{\nu-1}\left(B_{\tilde{\rho}}(y, 2 t)\right) \leq \mu \mathscr{H}^{\nu-1}\left(B_{\tilde{\rho}}(y, t)\right)
$$

для любой точки $y \in Q_{0}$ и шаров $B_{\tilde{\rho}}(y, 2 t) \subset Q_{0}$ с некоторой постоянной $\mu \in$ $(0, \infty)$, не зависящей от точки $y \in Q_{0}$ и шара $B_{\tilde{\rho}}(y, 2 t) \subset Q_{0}$.

3. Существуют постоянные $c_{11}, c_{12} \in(0, \infty)$ такие, что

$$
c_{11} \mathscr{H}^{\nu-1}(A) \leq \mathscr{H}^{N-1}(A) \leq c_{12} \mathscr{H}^{\nu-1}(A)
$$

для любого измеримого множества $A \subset Q_{0}$.

4. Квазиметрическое пространство $\left(Q_{0}, \tilde{\rho}\right)$ с мерой Хаусдорфа $\mathscr{H}^{\nu-1}$, удовлетворяющей условию (65), является пространством однородного типа (см. определение, например, в $[72,74])$.

5. Пространством однородного типа является также и метрическое пространство $\left(Q_{0}, \tilde{\rho}\right)$ с определенной на $Q_{0}$ мерой $\mathscr{H}^{N-1}$. 
ДокАЗАТЕЛьСтво. Так как ограничение квазиметрики на произвольное множество наследует свойства квазиметрики, утверждение 1 доказано.

Свойство удвоения меры вытекает из [75, теорема 3.17], где доказано, что $\mathscr{H}^{\nu-1}\left(B_{\tilde{\rho}}(z, r)\right) \sim r^{\nu-1}$ равномерно $^{11)}$ по всем $z \in Q_{0}$ и $B_{\tilde{\rho}}(z, r) \subset Q$.

Более того, из $[75$, теоремы $3.7,3.17]$ выводим, что мера Хаусдорфа $\mathscr{H}^{\nu-1}(A)$ множества $A \subset Q_{0}$ сравнима с мерой Хаусдорфа $\mathscr{H}^{N-1}(A):$ неравенства (66) вытекают из эквивалентностей

$$
\mathscr{H}^{\nu-1}\left(B_{\tilde{\rho}}(z, r)\right) \sim r^{\nu-1} \quad \text { и } \quad \mathscr{H}^{N-1}\left(B_{\tilde{\rho}}(z, r)\right) \sim r^{\nu-1},
$$

равномерных по $z \in Q_{0}$ и $B_{\tilde{\rho}}(z, r) \subset Q_{0}$ (см. [75, теоремы $\left.\left.3.7,3.17\right]\right)$.

Свойство 5 выводим из (66) и свойства 4.

ШАГ 3. Отображение $h: Q_{0} \times I_{k} \mapsto Q$, где $I_{k}=\left(-t_{k}, t_{k}\right)$ (см. (64)), определенное по правилу

$$
Q_{0} \times I_{k} \ni\left(z, y_{j}\right) \mapsto h\left(z, y_{j}\right)=\left(z, z \exp y_{j} X_{1 j}\right),
$$

является диффеоморфизмом. Можно подобрать $M>0$ в (63) такое, чтобы неравенства

$$
0<\varkappa_{1} \leq\left|\operatorname{det} h\left(z, y_{j}\right)\right| \leq \varkappa_{2}<\infty
$$

выполнялись для всех точек $\left(z, y_{j}\right) \in Q_{0} \times I_{k}$ с постоянными $\varkappa_{1}, \varkappa_{2} \in(0, \infty)$, не зависящими от $k \in \mathbb{N}$, где $k$ из (63).

$\mathrm{C}$ помощью отображения (68) интегрирование по области $Q$ можно перенести на открытое множество $Q_{0} \times I_{k}$. Рассмотрим на $Q_{0} \times I_{k}$ тензорное произведение $\Lambda$ меры $\mathscr{H}^{\nu-1}$ на $Q_{0}$ с мерой $\mathscr{H}^{1}$ на $I_{k}$. В силу соотношений (66) мера $\Lambda$ сравнима с мерой $\mathscr{H}^{N}$ :

$$
\varkappa_{3} \Lambda(E) \leq \mathscr{H}^{N}(E) \leq \varkappa_{4} \Lambda(E)
$$

для любого измеримого множества $E \subset Q_{0} \times I_{k}$. Постоянные $\varkappa_{3}, \varkappa_{4} \in(0, \infty)$ не зависят от выбора множества $E \subset Q_{0} \times I_{k}$. Следовательно, меры $\Lambda$ и $\mathscr{H}^{N}$ абсолютно непрерывны одна относительно другой. Поэтому существует производная Радона - Никодима $\mathfrak{D}(x)$ меры $\mathscr{H}^{N}$ по мере $\Lambda$, удовлетворяющая соотношениям

$$
\varkappa_{3} \leq \mathfrak{D}(x)=\frac{d \mathscr{H}^{N}}{d \Lambda}(x) \leq \varkappa_{4} \quad \text { для } \mathscr{H}^{N} \text {-п. в. } x \in Q_{0} \times I_{k} .
$$

Для функции $v(x)=|\operatorname{det} D h(x)| \cdot \mathfrak{D}(x)$ из $(69),(70)$ имеем оценки

$$
\varkappa_{1} \cdot \varkappa_{3} \leq v(x) \leq \varkappa_{2} \cdot \varkappa_{4} \quad \text { для } \mathscr{H}^{N} \text {-П. в. } x \in Q_{0} \times I_{k} .
$$

Если $u \in L_{1}(Q)$ - неотрицательная функция, то в силу (71) тем же свойством обладает произведение $u \cdot v$. Из вышеизложенного с помощью формулы

\footnotetext{
${ }^{11)} \mathrm{B}$ контексте настоящей работы величина $\mathscr{H}^{\nu-1}\left(B_{\tilde{\rho}}(z, r)\right)$ эквивалентна $r^{\nu-1}$ на $Q_{0}$ тогда и только тогда, когда существуют положительные числа $\zeta_{1}$ и $\zeta_{2}$ такие, что неравенство $\zeta_{1} r^{\nu-1} \leq \mathscr{H}^{\nu-1}\left(B_{\tilde{\rho}}(z, r)\right) \leq \zeta_{2} r^{\nu-1}$ выполняется для всех $z \in Q_{0}$ и всех $r$ таких, что $B_{\tilde{\rho}}(z, r) \subset$ $Q_{0}$.
} 
замены переменной (13) выводим

$$
\begin{gathered}
\int_{Q} u(y) d \mathscr{H}^{\nu}(y)=\int_{Q_{0} \times I_{k}} u(h(x))|\operatorname{det} D h(x)| d \mathscr{H}^{\nu}(x) \\
=\int_{Q_{0} \times I_{k}} u(h(x))|\operatorname{det} D h(x)| \cdot \mathfrak{D}(x) d \Lambda(x)=\int_{Q_{0}} d z \int_{I_{k}} u(h(x)) v(z, t) d t \\
=\int_{Q_{0}} d z \int_{I_{k}} u\left(z, \gamma_{z}(\tau)\right) v\left(h^{-1}\left(z, \gamma_{z}(\tau)\right)\right) d \mathscr{H}^{1}(\tau),
\end{gathered}
$$

где кривая $\gamma_{z}: I_{k} \rightarrow \mathbb{G}$, определенная условием $\gamma_{z}(\tau)=z \exp \tau X_{1 j}$, имеет касательный вектор $\left|\dot{\gamma}_{z}(\tau)\right|=1, \tau \in I_{k}$. (Здесь точки $Q_{0} \times I_{k}$ записаны как пары $(z, t), z_{0} \in Q_{0}, t \in I_{k}$, а точки $Q-$ как пары $\left.\left(z, \gamma_{z}(\tau)\right), z_{0} \in Q_{0}, \tau \in I_{k}.\right)$

ШАГ 4. С каждой точкой $z \in Q_{0}$ (см. (64)) и числом $r \in\left(0, t_{k}\right)$ таким, что $B_{\tilde{\rho}}(z, r) \subset Q_{0}$, ассоциируем трубчатую окрестность

$$
E(z, r)=\bigcup_{\tau \in\left(-t_{k}, t_{k}\right)} B_{c}\left(z \exp \tau X_{1 j}, r\right)
$$

Нас интересует поведение отношения

$$
\frac{\mathscr{H}^{\nu}(f(E(z, r)))}{r^{\nu-1}}
$$

при $r \rightarrow 0$. В [67, основная лемма] ([76, лемма 3]) на группах Гейзенберга (Карно) доказано, что верхний предел этого отношения конечен для п. в. $z \in$ $Q_{0}$ :

$$
\overline{\mathscr{V}}^{\prime}(z)=\varlimsup_{r \rightarrow 0} \frac{\mathscr{H}^{\nu}(f(E(z, r)))}{r^{\nu-1}}<\infty
$$

для $\mathscr{H}^{\nu-1}$-п. в. точек $z \in Q_{0}$ (здесь и далее $\left.\mathscr{V}(z, r)=\mathscr{H}^{\nu}(f(E(z, r)))\right)$. Таким образом, неравенство $\overline{\mathscr{V}}^{\prime}(z)<\infty$ выполняется во всех точках $z \in Q_{0} \backslash T^{\prime}$, где $T^{\prime} \subset Q_{0}-$ некоторое множество нулевой $\mathscr{H}^{\nu-1}$-меры.

ШАГ 5. На интервале $I_{k}=\left(-t_{k}, t_{k}\right)$ (см. (64)) возьмем произвольные дизъюнктные отрезки $\Delta_{1}=\left[a_{1}, b_{1}\right], \Delta_{2}=\left[a_{2}, b_{2}\right], \ldots, \Delta_{l}=\left[a_{l}, b_{l}\right]$ с длинами $b_{1}, b_{2}, \ldots, b_{l}$ такими, что и $b_{1}+b_{2}+\cdots+b_{l}<2 t_{k}$.

Фиксируем достаточно малое положительное число $r \in\left(0, t_{k}\right)$ такое, что $B_{\tilde{\rho}}(z, r) \subset Q_{0}$. С точкой $z$ и интервалом $\Delta_{i}$ ассоциируем открытое множество

$$
U_{i}(z, r)=\bigcup_{\tau \in \Delta_{i}} B_{c}\left(z \exp \tau X_{1 j}, r\right)
$$

Пусть $r>0$ выбрано настолько малым, что открытые множества $U_{1}(z, r), \ldots$, $U_{l}(z, r)$ дизъюнктны и $U_{i}(z, r) \subset Q, i=1, \ldots, l$ (см. (63) и (64)).

Рассмотрим континуум $F_{i}=\left\{z \exp \tau X_{1 j}: \tau \in \Delta_{i}\right\}$ и конденсатор $E_{i}=$ $\left(F_{i}, U_{i}(z, r)\right)$. По лемме 41 верна оценка

$$
\operatorname{cap}\left(E_{i} ; L_{p}^{1}\left(U_{i}(z, r) ; \omega\right)\right) \leq \frac{\omega\left(U_{i}(z, r)\right)}{r^{p}}=\frac{\int_{U_{i}(z, r)} \omega(y) d y}{r^{p}}, \quad i=1, \ldots, l .
$$

С другой стороны, при $\nu-1<p<\infty$ из леммы 42 вытекает, что

$$
c_{10}^{\frac{\nu-1}{p}} \frac{\operatorname{diam} f\left(F_{i}\right)}{\mathscr{H}^{\nu}\left(f\left(U_{i}(z, r)\right)\right)^{\frac{p-(\nu-1)}{p}}} \leq \operatorname{cap}^{\frac{\nu-1}{p}}\left(f\left(E_{i}\right) ; L_{p}^{1}\left(U_{i}\right)\right) .
$$


Из последних двух соотношений, учитывая неравенство (55), выводим, что

$$
\left.\operatorname{diam} f\left(F_{i}\right)\right) \leq\left(\frac{1}{c_{10}}\right)^{\frac{\nu-1}{p}} \frac{1}{r^{\nu-1}} \mathscr{H}^{\nu}\left(f\left(U_{i}(z, r)\right)\right)^{\frac{p-(\nu-1)}{p}} \omega\left(U_{i}(z, r)\right)^{\frac{\nu-1}{p}} .
$$

Суммируя неравенство (75) по $i=1, \ldots, l$, применяя неравенство Гёльдера и используя свойства квазиаддитивных функций, приходим к соотношениям

$$
\begin{gathered}
\sum_{i=1}^{l} \operatorname{diam} f\left(F_{i}\right) \leq\left(\frac{1}{c_{10}}\right)^{\frac{\nu-1}{p}} \frac{1}{r^{\nu-1}}\left(\sum_{i=1}^{l} \mathscr{H}^{\nu}\left(f\left(U_{i}(z, r)\right)\right)\right)^{\frac{p-(\nu-1)}{p}}\left(\sum_{i=1}^{l} \omega\left(U_{i}(z, r)\right)\right)^{\frac{\nu-1}{p}} \\
\leq c_{13} \cdot c_{14}\left(\frac{\mathscr{V}_{\nu}(z, r)}{r^{\nu-1}}\right)^{\frac{q-(\nu-1)}{q}}\left(\frac{\sum_{i=1}^{l} \omega\left(U_{i}(z, r)\right)}{r^{\nu-1}}\right)^{\frac{\nu-1}{p}}, \quad \text { (76) }
\end{gathered}
$$

где $c_{13}=\left(\frac{1}{c_{10}}\right)^{\frac{\nu-1}{p}}, c_{14}=\left(\beta_{\nu}(2 \alpha)^{\nu}\right)^{\frac{\nu-1}{p}}$, а постоянная $\beta_{\nu}(2 \alpha)^{\nu}$ будет определена ниже в $(81)$.

Заметим, что левая часть (76) не зависит от $r$. Поэтому перейти к пределу в правой части (76) можно по любой последовательности $r_{p} \rightarrow 0$ при $p \rightarrow \infty$. Устремляя $r \rightarrow 0$, докажем следующее неравенство, справедливость которого при $\mathscr{H}^{\nu-1}$-п. в. $z \in Q_{0}$ обусловлена существованием пределов в двух скобках выражения (76) для $\mathscr{H}^{\nu-1}$-п. в. $z \in Q_{0}$ (см. детали после формулы (77)):

$$
\sum_{i=1}^{l} \operatorname{diam} f\left(F_{i}\right) \leq l c_{13} \cdot c_{14}\left(\overline{\mathscr{V}}^{\prime}(z)\right)^{\frac{p-(\nu-1)}{p}}\left(\bigcup_{i=1}^{l} \omega(z, \tau) d \gamma_{z}(\tau)\right)^{\frac{\nu-1}{p}},
$$

где $\Delta_{i} \ni \tau \mapsto \gamma_{z}(\tau)=z \exp \left(\tau X_{1 j}\right)$. Первое частное в скобках (см. (76)) имеет конечный предел (73) во всех точках $z \in Q_{0} \backslash T^{\prime}$, где $T^{\prime}$ имеет $\mathscr{H}^{\nu-1}$-меру нуль (это оговорено на шаге 4).

Докажем, что второй сомножитель выражения (76) имеет конечный предел, указанный в выражении (77), при $\mathscr{H}^{\nu-1}$-п. в. $z \in Q_{0}$ для некоторой последовательности $r_{p} \rightarrow 0$ при $p \rightarrow \infty$.

Чтобы в этом убедиться, рассмотрим отдельное слагаемое во втором сомножителе формулы (76), например, с номером $i$ (из существования предела для каждого слагаемого получаем существование предела и для конечного набора слагаемых). Вспоминая определение множества $U_{i}(z, r)$ в $(74)$, имеем

$$
\omega\left(U_{i}(z, r)\right)=\int_{U_{i}(z, r)} \omega(y) d y
$$

Для достаточно малого $r>0$ положим $\tau_{m}=a_{i}+m r$ при $m=0,1, \ldots, m_{r}$, где $m_{r}-$ наименьшее натуральное число такое, что $\tau_{m_{r}} \geq b_{i}$. Для произвольного $\zeta \in\left(\tau_{m-1}, \tau_{m}\right)$ и некоторого $\zeta_{m} \in\left(\tau_{m-1}, \tau_{m}\right)$, выбираемого ниже в формуле (79), получаем включения

$$
B_{c}\left(z \exp \zeta X_{1 j}, r\right) \subset B_{c}\left(z \exp \zeta_{m} X_{1 j}, 2 r\right) \subset B_{\rho}\left(z \exp \zeta_{m} X_{1 j}, 2 \alpha r\right),
$$

где $\alpha-$ положительная постоянная такая, что $B_{c}(y, r) \subset B_{\rho}(y, \alpha r)$ для всех $y \in \mathbb{G}$ и $\rho>0$.

При фиксированном $r$ для любого $m=1,2,3, \ldots, m_{r}$ имеем равенства

$$
\frac{1}{r} \int_{\tau_{m-1}}^{\tau_{m}} \omega\left(B_{\rho}\left(z \exp \tau X_{1 j}, 2 \alpha r\right)\right) d \tau=\omega\left(B_{\rho}\left(z \exp \zeta_{m} X_{1 j}, 2 \alpha r\right)\right),
$$


поскольку функция $\tau \mapsto \omega\left(B_{\rho}\left(z \exp \tau X_{1 j}, 2 \alpha r\right)\right)$ непрерывна $\left(\zeta_{m} \in\left(\tau_{m-1}, \tau_{m}\right)-\right.$ число, существование которого обусловлено теоремой о среднем). При таком выборе $\left\{\zeta_{m}\right\}, m=1,2, \ldots, m_{r}$, из (74) и (78) выводим включение

$$
U_{i}(z, r) \subset \bigcup_{m} B_{\rho}\left(z \exp \zeta_{m} X_{1 j}, 2 \alpha r\right),
$$

где объединение идет по $m$, указанным выше. Отсюда с учетом (79) приходим к соотношениям

$$
\omega\left(U_{i}(z, r)\right) \leq \sum_{m} \omega\left(B_{\rho}\left(z \exp \zeta_{m} X_{1 j}, 2 \alpha r\right)\right)=\frac{1}{r} \int_{a_{i}}^{\tau_{m_{r}}} \omega\left(B_{\rho}\left(z \exp \tau X_{1 j}, 2 \alpha r\right)\right) d \tau
$$

Из (80) выводим

$$
\frac{\omega\left(U_{i}(z, r)\right)}{r^{\nu-1}} \leq \beta_{\nu}(2 \alpha)^{\nu} \int_{a_{i}}^{\tau_{m_{r}}} \frac{\omega\left(B_{\rho}\left(z \exp \tau X_{1 j}, 2 \alpha r\right)\right)}{\mathscr{H}^{N}\left(B_{\rho}(e, 2 \alpha r)\right)} d \tau,
$$

где $\beta_{\nu}=\mathscr{H}^{N}\left(B_{\rho}(e, 1)\right)$ - объем шара $B_{\rho}(e, 1)$. Подынтегральное выражение в (81) представляет собой значение функции

$$
\omega_{r}(y)=\frac{1}{\mathscr{H}^{N}\left(B_{\rho}(e, 2 \alpha r)\right)} \int_{B_{\rho}(y, 2 \alpha r)} \omega(w) d w
$$

в точке $y=z \exp \tau X_{1 j}$, а сама функция $\omega_{r}(y)$ - это аналог среднего по Стеклову функции $\omega(y)$ на группе Карно. Известно (см., например, [70, предложение 1.20$])$, что

$$
\left\|\omega_{r}(\cdot)-\omega(\cdot) \mid L_{1}(Q)\right\| \rightarrow 0 \quad \text { при } r \rightarrow 0 .
$$

Более того, применяя формулу (72), приходим к сходимости

$$
\int_{I_{k}}\left|\omega_{r_{p}}\left(z \exp \tau X_{1 j}\right)-\omega\left(z \exp \tau X_{1 j}\right)\right| d \tau \rightarrow 0
$$

для некоторой последовательности $r_{p} \rightarrow 0$, когда $p \rightarrow \infty$, для всех $z \in Q_{0} \backslash$ $\Sigma_{j}$, где $\mathscr{H}^{\nu-1}\left(\Sigma_{j}\right)=0$. Отсюда, в частности, имеем, что функция $I_{k} \ni \tau \mapsto$ $\omega\left(z \exp \tau X_{1 j}\right)$ суммируема на $I_{k}$ для всех $z \in Q_{0} \backslash \Sigma_{j}$.

Очевидно, что сходимость (82) имеет место также на любом интервале $\left(a_{i}, b_{i}+\delta_{i}\right) \subset I_{k}$ (здесь $\delta_{i}-$ произвольное число из промежутка $\left.\left(0, t_{k}-b_{i}\right)\right)$, а функция $\left(a_{i}, b_{i}+\delta_{i}\right) \ni \tau \mapsto \omega\left(z \exp \tau X_{1 j}\right)$ суммируема на $\left(a_{i}, b_{i}+\delta_{i}\right)$ для всех $z \in Q_{0} \backslash \Sigma_{j}$.

На основании изложенного из (81), (82) для всех $z \in Q_{0} \backslash \Sigma_{j}$ получаем

$$
\begin{aligned}
\varlimsup_{p \rightarrow \infty} \frac{\omega\left(U_{i}\left(z, r_{p}\right)\right)}{r_{p}^{\nu-1}} \leq \beta_{\nu}(2 \alpha)^{\nu} \lim _{p \rightarrow \infty} \int_{a_{i}}^{b_{i}+\delta} \omega_{r_{p}}\left(z \exp \tau X_{1 j}\right) d \tau & \\
& =\beta_{\nu}(2 \alpha)^{\nu} \int_{a_{i}}^{b_{i}+\delta} \omega\left(z \exp \tau X_{1 j}\right) d \tau
\end{aligned}
$$

для любого числа $\delta \in\left(0, \delta_{i}\right)$ (здесь принято во внимание, что $\tau_{m_{r}} \geq b_{i}$ и $\tau_{m_{r}} \rightarrow b_{i}$ при $r \rightarrow 0$ ). Учитывая произвол в выборе $\delta>0$ и абсолютную непрерывность 
интеграла Лебега, из (83) выводим

$$
\varlimsup_{p \rightarrow \infty} \frac{\omega\left(U_{i}\left(z, r_{p}\right)\right)}{r_{p}^{\nu-1}} \leq \beta_{\nu}(2 \alpha)^{\nu} \int_{a_{i}}^{b_{i}} \omega\left(z \exp \tau X_{1 j}\right) d \tau .
$$

С учетом того, что количество слагаемых во втором сомножителе (76) конечное, неравенство (77) доказано для всех точек $z \in Q_{0} \backslash\left(T^{\prime} \cup \Sigma_{j}\right)$, где объединение $T^{\prime} \cup \Sigma_{j} \subset Q_{0}$ имеет $\mathscr{H}^{\nu-1}$-меру нуль.

ШАГ 6. Неравенство (77) показывает также, что абсолютная непрерывность отображения $f: \gamma_{z} \rightarrow D$ при фиксированном $z$ обеспечена абсолютной непрерывностью интеграла

$$
\int_{I_{k}} \omega\left(z \exp \tau X_{1 j}\right) d \tau
$$

на промежутке $\left\{z \exp t X_{1 j}: t \in I_{k}\right\}$ интегральной линии векторного поля $X_{1 j}$. Следовательно, неравенство (77) можно распространить на произвольный счетный дизъюнктный набор отрезков $\Delta_{i} \subset I_{k}$.

Так как $j$ может быть произвольным натуральным числом от 1 до $n$, абсолютная непрерывность отображения $f: D^{\prime} \rightarrow D$ доказана. Отсюда с учетом (57) доказано также, что $f \in W_{1, \text { loc }}^{1}\left(D^{\prime}\right)$ (см. детали в [73, предложение 4.2]).

4.5. Применения. В работе [18] сформулирована следующая

Теорема 43 [18, теорема 9]. Пусть $D$ и $D^{\prime}$ - области на группе Карно. Предположим, что гомеоморфизм $\varphi: D \rightarrow D^{\prime}$ индуцирует ограниченный оператор композиции $\varphi^{*}: L_{p}^{1}\left(D^{\prime}\right) \cap \operatorname{Lip}_{l}\left(D^{\prime}\right) \rightarrow L_{q}^{1}(D), \nu-1<q \leq p<\infty$. Тогда обратное отображение $\varphi^{-1}: D^{\prime} \rightarrow D$ индуцирует ограниченный оператор композиции $\varphi^{-1 *}: L_{r}^{1}(D) \cap \operatorname{Lip}_{l}(D) \rightarrow L_{s}^{1}\left(D^{\prime}\right)$, где $r=\frac{q}{q-(\nu-1)}$ и $s=\frac{p}{p-(\nu-1)}$.

Однако ее доказательство, приведенное в [18], содержит пробелы, перешедшие из [17], которые устранимы с помощью результатов $\S 4$. (Пробел из [17] устранен и в [20, теорема 6] и, другим методом, в теореме 23 настоящей работы.)

Действительно, если гомеоморфизм $\varphi: D \rightarrow D^{\prime}$ индуцирует ограниченный оператор композиции $\varphi^{*}: L_{p}^{1}\left(D^{\prime}\right) \cap \operatorname{loc}_{l}\left(D^{\prime}\right) \rightarrow L_{q}^{1}(D)$, то $\varphi \in L_{q, \mathrm{loc}}^{1}(D)$ и $\varphi$ имеет конечное искажение (см. [18, предложение 1 , теорема 2]). В силу утверждения 40 обратное отображение $\varphi^{-1}$ принадлежит $W_{1, \mathrm{loc}}^{1}\left(D^{\prime}\right)$ и имеет конечное искажение. Последнее свойство является необходимым для того, чтобы $\varphi^{-1}: D^{\prime} \rightarrow D$ индуцировало ограниченный оператор композиции $\varphi^{-1 *}: L_{r}^{1}(D) \cap \operatorname{Lip}_{l}(D) \rightarrow L_{s}^{1}\left(D^{\prime}\right)$, где $r=\frac{q}{q-(\nu-1)}$ и $s=\frac{p}{p-(\nu-1)}$. В [18] доказательство конечности искажения $\varphi^{-1}$ не приводится.

4.6. Обобщение. Метод из $\S 4$ применим также для обобщения теорем 21 и 23 на непрерывные открытые дискретные отображения групп Карно.

Благодарность. Выражаю искреннюю благодарность рецензенту за критическое рассмотрение рукописи и комментарии, которые помогли улучшить первоначальный вариант работы.

\section{ЛИТЕРАТУРА}

1. Hencl S., Koskela P. Regularity of the inverse of a planar Sobolev homeomorphism // Arch. Rat. Mech. Anal. 2006. V. 180. P. 75-95. 
2. Onninen $J$. Regularity of the inverse of spatial mappings with finite distortion // Calc. Var. and PDE. 2006. V. 26, N 3. P. 331-341.

3. Ball J. M. Global invertibility of Sobolev functions and the interpenetration of matter // Proc. Roy. Soc. Edinburgh Sect. A. 1981. V. 88, N 3-4. P. 315-328.

4. Menchoff D. Sur les différentielles totales des fonctions univalentes // Math. Ann. 1931. V. 105, N 1. P. $75-85$.

5. Gehring F. W., Lehto O. On the total differentiability of functions of a complex variable // Ann. Acad. Sci. Fenn. Ser. AI. 1959. V. 272. P. 1-9.

6. Martio O., Ryazanov O. , Srebro U., Yakubov E. Moduli in modern mapping theory. New York: Springer-Verl., 2008.

7. Водопьянов C. K. Основы квазиконформного анализа двухиндексной шкалы пространственных отображений // Докл. АН. 2019. Т. 484, № 2. С. 142-146.

8. Водопьянов C. К. Основы квазиконформного анализа двухиндексной шкалы пространственных отображений // Сиб. мат. журн. 2018. Т. 59, № 5. С. 1020-1056.

9. Водопьянов С. К. О дифференцируемости отображений класса Соболева $W_{n-1}^{1}$ с условиями на функцию искажения // Сиб. мат. журн. 2018. Т. 59, № 6. С. 1240-1267.

10. Водопьянов C. K. Операторы композиции в весовых пространствах Соболева и теория $\mathscr{Q}_{p}$-гомеоморфизмов .. Докл. АН. Математика, информатика, процессы управления. 2020. T. 494, № 5. C. 21-25. DOI: 10.31857/S268695432005046X.

11. Водопьянов C. К. Об аналитических и геометрических свойствах отображений в теории

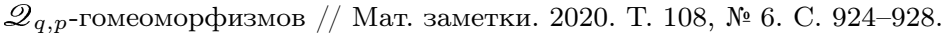

12. Водопьянов C. К., Томилов А. О. Функциональные и аналитические свойства одного класса отображений квазиконформного анализа // Изв. РАН. Cер. мат. 2021. Т. 85. DOI: 10.4213/im9082.

13. Водопьянов C. К. Формула Тейлора и функциональные пространства. Новосибирск: НГУ, 1988.

14. Водопьянов C. К. Отображения однородных групп и вложения функциональных пространств. // Сиб. мат. журн. 1989. Т. 30, № 5. С. 25-41.

15. Водопьянов C. K. Весовые пространства Соболева и теория отображений // Всесоюз. математическая шк. «Теория потенциала» Кацивели, 26 июня - 3 июля 1991 г. Тез. докл. Киев: Ин-т математики АН УССР, 1991. С. 7.

16. Водопьянов C. К. Геометрические аспекты пространств обобщенно-дифференцируемых функций. Автореф. дис. ... докт. физ.-мат. наук. Новосибирск: Ин-т математики СО PAH, 1992.

17. Ухлов А. Д. Отображения, порождающие вложения пространств Соболева // Сиб. мат. журн. 1993. Т. 34, № 1. С. 185-192.

18. Водопьянов C. К., Ухлов А. Д. Пространства Соболева и $(P, Q)$-квазиконформные отображения групп Карно // Сиб. мат. журн. 1998. Т. 39, № 4. С. 776-795.

19. Водопьянов C. К., Ухлов А. Д. Операторы суперпозиции в пространствах Соболева // Изв. вузов. Математика. 2002. № 10. С. 11-33.

20. Водопьянов C. К. О регулярности отображений, обратных к соболевским // Мат. сб. 2012. Т. 203, № 10. С. 3-32.

21. Соболев С. Л. О некоторых группах преобразований $n$-мерного пространства // Докл. AH. 1941. T. 32, № 6. C. 380-382.

22. Мазья В. Г. Классы множеств и теоремы вложения функциональных классов. Некоторые проблемы теории эллиптических операторов. Автореф. дис. ... канд. физ.-мат. наук. Л.: Ленингр. ун-т, 1961.

23. Решетняк Ю. Г. Пространственные отображения с ограниченным искажением. Новосибирск: Наука, 1982.

24. Mostow G. D. Quasi-conformal mappings in $n$-space and the rigidity of hyperbolic space forms // Inst. Hautes Études Sci. Publ. Math. 1968. V. 34, N 1. P. 53-104.

25. Reiman $M$. Über harmonishe Kapazität und quasikonforme Abbildungen in Raum // Comm. Math. Helv. 1969. V. 44. P. 284-307.

26. Väisälä $J$. Lectures on $n$-dimensional quasiconformal mappings. Berlin: Springer-Verl., 1971. (Lecture Notes in Math.; V. 229).

27. Gehring $F$. W. Lipschitz mappings and the $p$-capacity of rings in $n$-space // Advances in the theory of Riemann surfaces. Princeton: Princeton Univ. Press, 1971. P. 175-193.

28. Lelong-Ferrand $J$. Etude d'une classe d'applications liées à des homomorphismes d'algèbres de fonctions et généralisant les quasi-conformes // Duke Math. 1973. V. 40. P. 163-186. 
29. Водопьянов C. К., Гольдштейн В. М. Структурные изоморфизмы пространств $W_{n}^{1}$ и квазиконформные отображения // Сиб. мат. журн. 1975. Т. 16, № 2. С. 224-246.

30. Водопьянов C. К., Гольдштейн В. М. Функциональные характеристики квазиизометрических отображений // Сиб. мат. журн. 1976. Т. 17, № 4. С. 768-773.

31. Романов А. С. О замене переменной в пространствах потенциалов Бесселя и Рисса // Функциональный анализ и математическая физика. Новосибирск: Ин-т математики СО AH, 1985. C. $117-133$.

32. Водопьянов C. K. $L_{p}$-теория потенциала и квазиконформные отображения на однородных группах // Современные проблемы геометрии и анализа. Новосибирск: Наука, 1989. C. $45-89$.

33. Vodopyanov S. K. Composition operators on Sobolev spaces // Complex analysis and dynamical systems. II: A conf. in honor of Professor Lawrence Zalcman's Sixtieth Birthday, June 9-12, 2003, Nahariya, Israel. Eds: M. Agranovsky, L. Karp, D. Shoikhet. Ann Arbor: Amer. Math. Soc., 2005. P. 327-342. (AMS Contemp. Math.; V. 382).

34. Водопьянов C. K., Евсеев Н. А. Изоморфизмы соболевских пространств на группах Карно и квазиизометрические отображения // Сиб. мат. журн. 2014. Т. 55, № 5. С. 1001-1039.

35. Водопьянов C. К., Евсеев Н. А. Изоморфизмы соболевских пространств на группах Карно и метрические свойства отображений // Докл. АН. 2015. Т. 464, № 2. С. 131-135.

36. Водопьянов C. К., Евсеев Н. А. Изоморфизмы соболевских пространств на группах Карно и квазиконформные отображения // Сиб. мат. журн. 2015. Т. 56, № 5. С. 989-1029.

37. Водопьянов C. К. О допустимых заменах переменных для функций классов Соболева на (суб)римановых многообразиях // Докл. АН. 2016. Т. 468, № 6. С. 609-613.

38. Водопьянов С. К. Допустимые замены переменных для функций классов Соболева на (суб)римановых многообразиях // Мат. сб. 2019. Т. 210, № 1. С. 63-112.

39. Водопьянов C. К. Изоморфизмы соболевских пространств на римановых многообразиях и квазиконформные отображения // Сиб. мат. журн. 2019. Т. 60, № 5. С. 996-1034.

40. Molchanova A., Vodop'yanov S. Injectivity almost everywhere and mappings with finite distortion in nonlinear elasticity // Calculus of Variations and PDE. 2020. V. 59, N 1. P. DOI: 10.1007/s00526-019-1671-4 (on-line first).

41. Байкин А. Н., Водопьянов С. К. Емкостные оценки, теоремы типа Лиувилля и об устранении особенностей для отображений с ограниченным $(p, q)$-искажением // Сиб. мат. журн. 2015. Т. 56, № 2. С. 290-321.

42. Ukhlov A. D., Vodop'yanov S. K. Mappings associated with weighted Sobolev spaces // Complex analysis and dynamical systems. III. Ann Arbor; Providence: Amer. Math. Soc., 2008. P. 369-382. (AMS Contemp. Math; V. 455).

43. Koskela P., Panka P., Zhang Yi Ru-Ya. Ahlfors reflection theorem for $p$-morphisms. arXiv: $1912.09200 \mathrm{v} 2$.

44. Ambrosio L., Tilli P. Topics on analysis in metric spaces. New York: Oxford Univ. Press, 2004.

45. Rado I., Reichelderfer P. V. Continuous transformations in analysis with an introduction to algebraic topology. Berlin: Springer-Verl., 1955.

46. Водопьянов C. K., Ухлов А. Д. Операторы суперпозиции в пространствах Лебега и дифференцируемость квазиаддитивных функций множества // Владикавказ. мат. журн. 2002. T. 4, № 1. C. 11-33.

47. Vodop'yanov $S$. K., Ukhlov A. D. Set functions and their applications in the theory of Lebesgue and Sobolev spaces. I // Sib. Adv. Math. 2004. V. 14, N 4. P. 78-125.

48. Rickman S. Quasiregular mappings. Berlin etc.: Springer-Verl., 1993. (Universitext).

49. Federer H. Geometric measure theory. New York: Springer-Verl., 1960.

50. Эванс Л. К., Гариепи Р. Ф. Теория меры и тонкие свойства функций. Новосибирск: Науч. книга, 2002.

51. Мазья В. Г. Пространства С. Л. Соболева. Л.: Изд-во Ленингр. ун-та, 1985.

52. Vodopyanov S. K. Moduli inequalities for $W_{n-1, \text { loc }}^{1}$-mappings with weighted bounded $(q, p)$ distortion // Complex Variables and Elliptic Equations. 2020. V. 65. https://doi.org/10.1080/ 17476933.2020.1825396.

53. Hajłasz $P$. Change of variables formula under minimal assumptions // Colloquium mathematicum. 1993. V. LXIV, N 1. P. 93-101.

54. Решетняк Ю. Г. Некоторые геометрические свойства функций и отображений с обобщенными производными // Сиб. мат. журн. 1966. Т. 7, № 4. С. 886-919. 
55. Martio O., Malý J. Lusin's condition $(N)$ and mappings of the class $W^{1, n} / /$ J. Reine Angew. Math. NN. 1995. V. 48, N 5. P. 19-36.

56. Водопьянов С. К. О дифференцируемости отображений классов Соболева на группе Карно // Мат. сб. 2003. Т. 194, № 6. С. 67-86.

57. Шварцман П. А. Теоремы продолжения для пространств функций, определяемых локальными приближениями. Автореф. дис. ... канд. физ.-мат. наук. Ярославль: Ярослав. ун-т, 1983.

58. Martio O., Rickman S., Väisälä J. Definitions for quasiregular mappings // Ann. Acad. Sci. Fenn. Math. 1969. V. 448. P. 1-40.

59. Кругликов В. И. Емкости конденсаторов и пространственные отображения, квазиконформные в среднем // Мат. сб. 1986. Т. 130, № 2. С. 185-206.

60. Brakalova M. A., Jenkins J. A. On solutions of the Beltrami equation // J. d'Anal. Math. 1998. V. 76. P. 67-92.

61. Салимов Р. Р. Абсолютная непрерывность на линиях и дифференцируемость одного обобщения квазиконформных отображений // Изв. РАН. Сер. мат. 2008. Т. 72, № 5. C. $141-148$.

62. Salimov R., Sevost'yanov E. ACL and differentiability of open discrete ring $(p, Q)$-mappings // Mat. Studii. 2011. V. 35. P. 28-36.

63. Трохимчук Ю. Ю. Непрерывные отображения и условия моногенности. М.: Физматгиз, 1963.

64. Afanas'eva O. S., Ryazanov V. I., Salimov R. R. Toward the theory of the Sobolev classes with critical exponent // Dopov. Nac. akad. nauk Ukr. 2019. V. 8. P. 3-8.

65. Tengvall $V$. Differentiability in the Sobolev space $W^{1, n-1} / /$ Ann. Acad. Sci. Fenn. Ser. AI. 2014. V. 51, N 12. P. 381-399.

66. Mostow G. D. Quasi-conformal mappings in $n$-space and the rigidity of hyperbolic space forms // Inst. Hautes Études Sci. Publ. Math. 1968. V. 34. P. 53-104.

67. Korányi A., Reimann H. M. Foundations for the theory of quasiconformal mappings on the Heisenberg group // Adv. Math. 1995. V. 111. P. 1-87.

68. Menshoff $D$. Sur les conditions suftisantes pour qu'une fonction univalente soit holomorphe // Мат. сб. 1933. V. 40, N 1. P. 3-23.

69. Pansu $P$. Métriques de Carnot-Carathéodory et quasiisométries des espacies symétriques de rang un // Ann. Math. 1989. V. 129, N 2. P. 1-60.

70. Folland G. B., Stein E. M. Hardy spaces on homogeneous groups. Princeton, NJ: Princeton Univ. Press, 1982. (Math. Notes; V. 28).

71. Bonfiglioli A., Lanconelli E. Uguzzoni F. Stratified Lie groups and potential theory for their sub-Laplacians. Berlin; Heidelberg: Springer-Verl., 2007. (Universitext).

72. Stein E. M. Harmonic analysis: real-variable methods, orthogonality, and oscillatory integrals. Princeton, NJ: Princeton Univ. Press, 1993. (Universitext).

73. Vodop'yanov S. K. P्P-differentiability on Carnot groups in different topologies and related topics // Proc. on analysis and geometry. Novosibirsk: Sobolev Inst. Press, 2000. P. 603-670.

74. Гусман М. Дифференцирование интегралов в $\mathbb{R}^{n}$. М.: Мир, 1978.

75. Karmanova M., Vodopyanov S. A coarea formula for smooth contact mappings of Carnot-Carathéodory spaces // Acta Appl. Math. 2013. V. 128. P. 67-111.

76. Водопьянов С. К., Грешнов А. В. Аналитические свойства квазиконформных отображений на группах Карно // Сиб. мат. журн. 1995. Т. 36, № 6. С. 1317-1327.

Поступила в редакчию 18 июля 2020 г.

После доработки 26 сентября 2020 г.

Принята к публикачии 9 октября 2020 г.

Водопьянов Сергей Константинович

Институт математики им. С. Л. Соболева СО РАН, пр. Академика Коптюга, 4, Новосибирск 630090

vodopis@math.nsc.ru 